( $* * * * * * * * * * * * * * * * * * * * * * * * * * * * * * * * * * * * * * * * * * * * * * * * * * * * * * * * * * * * * * * * * * * * * * * * * * * * * * * * * * * * * * *)$

(* This Mathematica notebook contains trials of fitting qPCR data with an exponential model.

(* The ' $\mathrm{d} 0$ ' values resulting from these trials are used to develop the plots in figure 3 of the MAK2 paper. *)

(*

$(* * * * * * \quad$ Written by Gregory Boggy (November 2009)

$(* * * * * * * * * * * * * * * * * * * * * * * * * * * * * * * * * * * * * * * * * * * * * * * * * * * * * * * * * * * * * * * * * * * * * * * * * * * * * * * * * * * * * * *)$

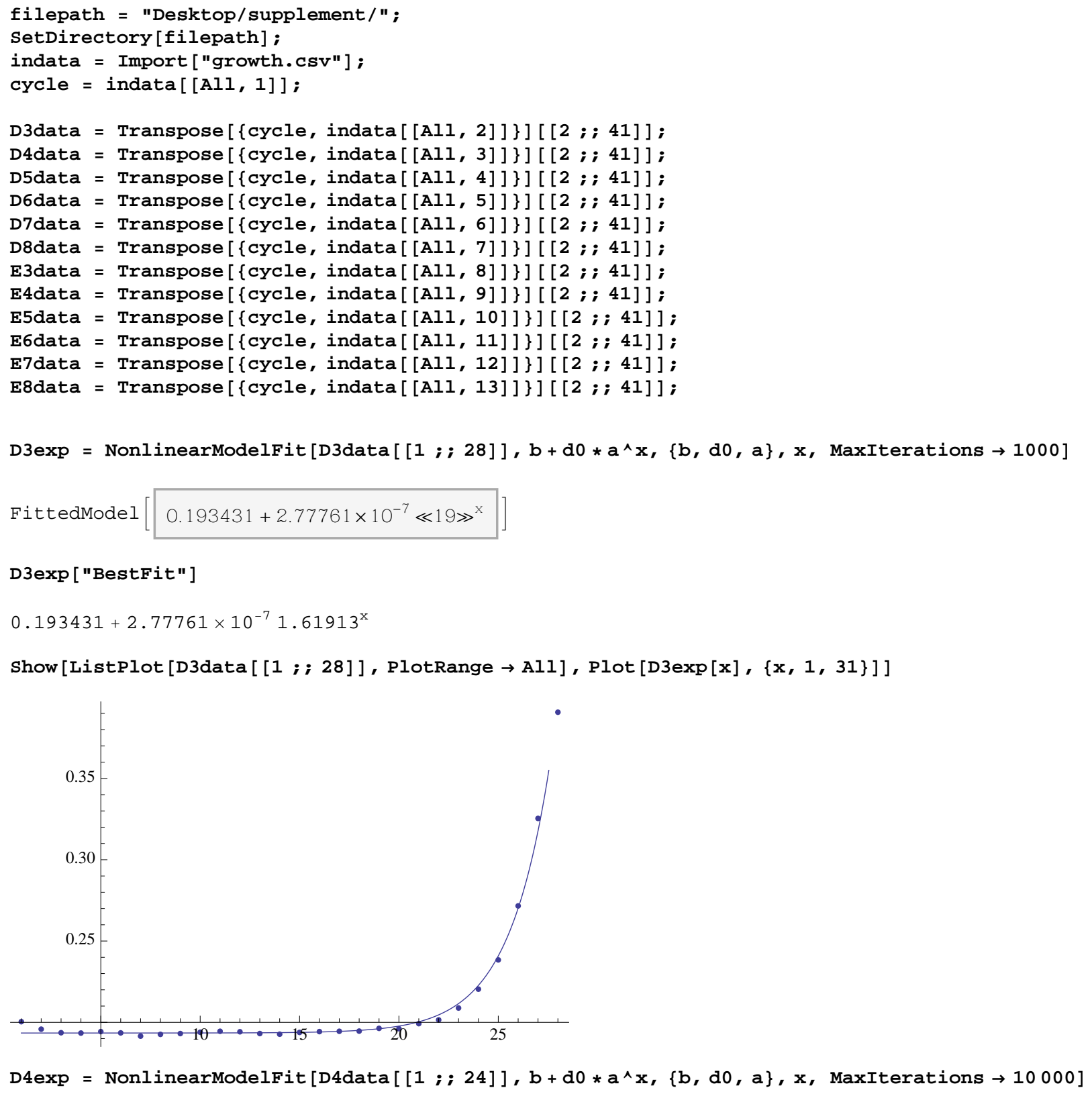

D4exp $=$ NonlinearModelFit $\left[\right.$ D4data $\left.[[1 ; ; 24]], b+d 0 * a^{\wedge} \mathbf{x},\{b, d 0, a\}, \mathbf{x}, \operatorname{MaxIterations} \rightarrow 10000\right]$

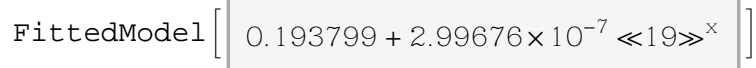




\section{D4exp ["BestFit"]}

$0.193799+2.99676 \times 10^{-7} 1.74385^{x}$

Show [ListPlot [D4data $[[1 ; ; 24]], P l o t R a n g e \rightarrow A l l], P l o t[D 4 \exp [x],\{x, 1,28\}]]$

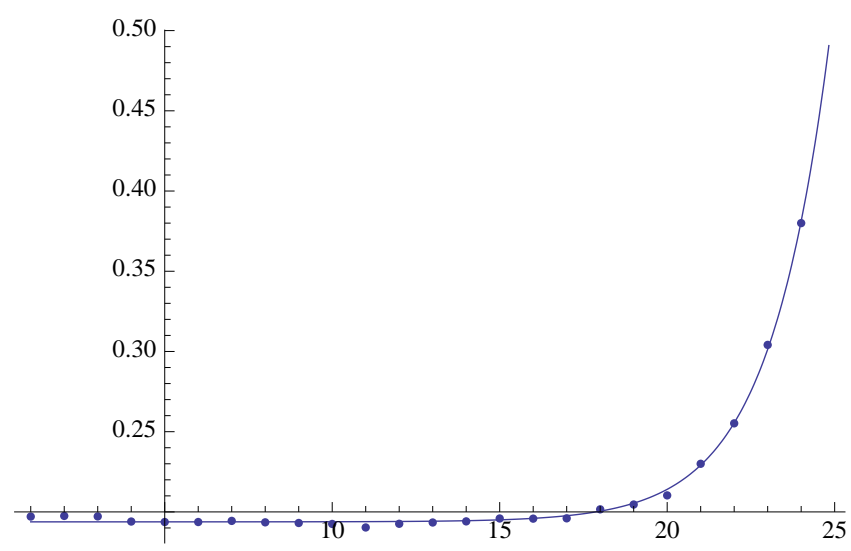

D5exp $=$ NonlinearModelFit $[$ D5data $\left.[1 ; ; ; 20]], b+d 0 * a^{\wedge} \mathbf{x},\{b, d 0, a\}, \mathbf{x}, \operatorname{MaxIterations} \rightarrow 10000\right]$ FittedModel $\left[0.195746+1.08399 \times 10^{-6} \ll 18 \gg^{x}\right]$

D5exp ["BestFit"]

$0.195746+1.08399 \times 10^{-6} 1.82639^{x}$

Show [ListPlot [D5data $[[1 ; 20]]$, PlotRange $\rightarrow A l l], P l o t[D 5 e x p[x],\{x, 1,23\}]]$

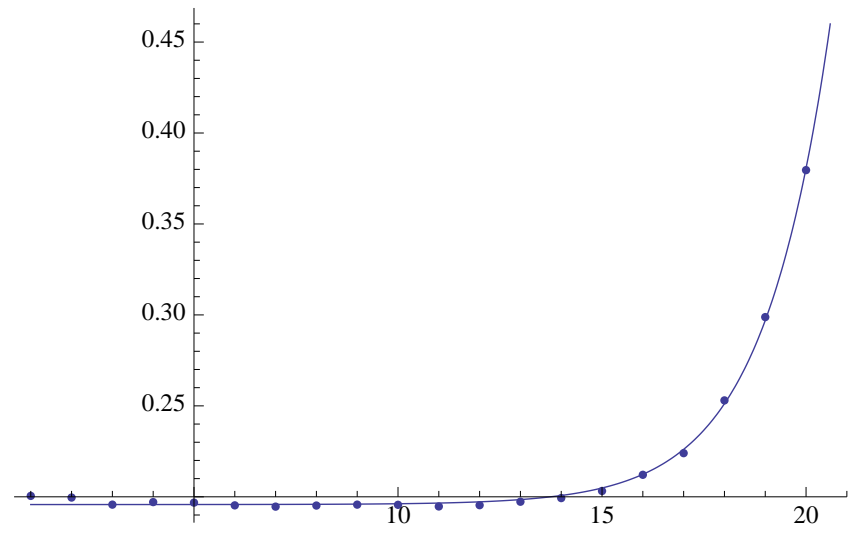

D6exp $=$ NonlinearModelFit $\left[\right.$ D6data $[[1 ; ; 17]], b+d 0 * a^{\wedge} \mathbf{x},\{b, d 0, a\}, \mathbf{x}$, MaxIterations $\left.\rightarrow 10000\right]$ FittedModel $\left[0.191898+4.55187 \times 10^{-6} \ll 19 \gg^{x}\right]$

D6exp [ BestFit"]

$0.191898+4.55187 \times 10^{-6} 1.86618^{x}$ 
Show [ListPlot [D6data $[[1 ; ; 17]], \mathrm{PlotRange} \rightarrow \mathrm{All}], \mathrm{Plot}[\mathrm{D} 6 \exp [\mathbf{x}],\{\mathbf{x}, 1,19\}]]$

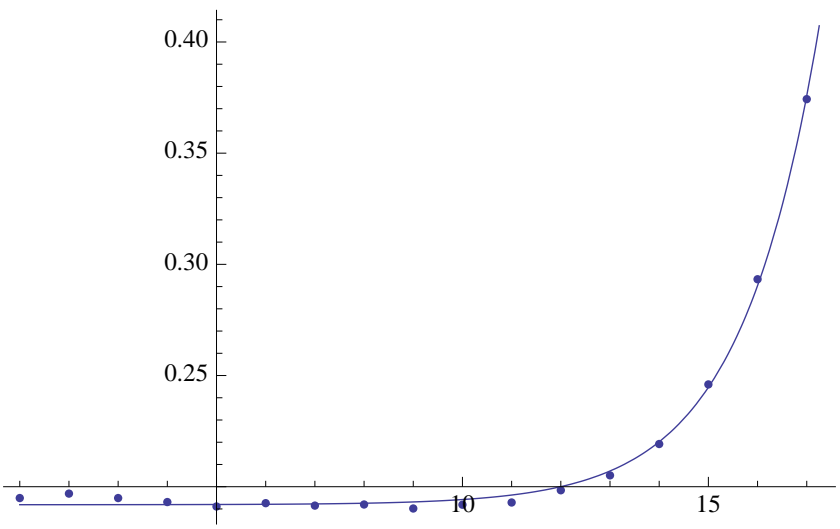

D7exp $=$ NonlinearModelFit $\left[D 7 d a t a[[1 ; ; 13]], b+d 0 * a^{\wedge} \mathbf{x},\{b, d 0, a\}, \mathbf{x}, \operatorname{MaxIterations} \rightarrow 1000\right]$ FittedModel $\left[0.196345+0.0000262309 \ll 19 \gg^{x}\right]$

D7exp ["BestFit"]

$0.196345+0.00002623091 .95542^{x}$

Show [ListPlot $[D 7$ data $[[1 ; ; 13]], \operatorname{PlotRange~} \rightarrow A l 1], \operatorname{Plot}[D 7 \exp [\mathbf{x}],\{\mathbf{x}, 1,15\}]]$

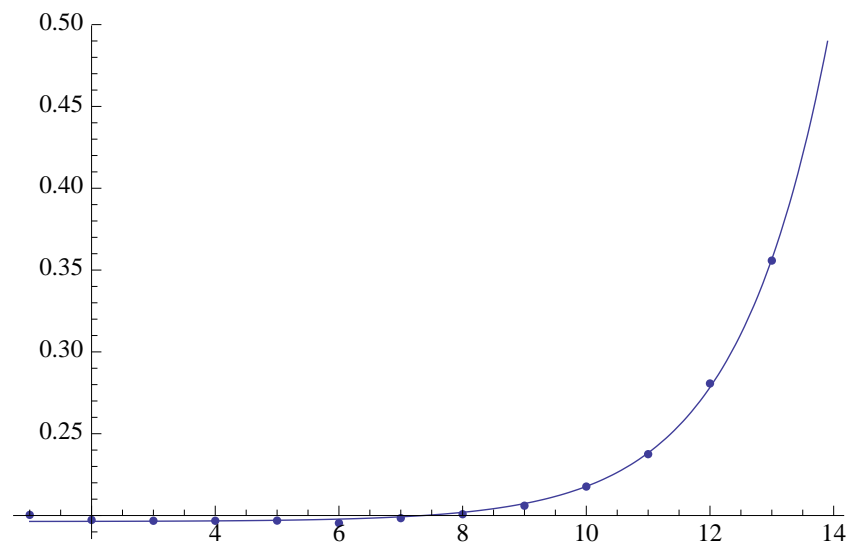

D8exp $=$ NonlinearModelFit $\left[\right.$ D8data $[[1 ; ; 10]], b+d 0 * a^{\wedge} \mathbf{x},\{b, d 0, a\}, \mathbf{x}$, MaxIterations $\left.\rightarrow 10000\right]$ FittedModel $\left[0.194705+0.000267799 \ll 19 \gg^{x}\right]$

D8exp [ BestFit"]

$0.194705+0.0002677991 .94344^{x}$ 
Show [ListPlot [D8data $[1 ; ; 10]], \mathrm{PlotRange} \rightarrow \mathrm{All}], \mathrm{Plot}[\mathrm{D} 8 \exp [\mathbf{x}],\{\mathbf{x}, 1,10\}]]$

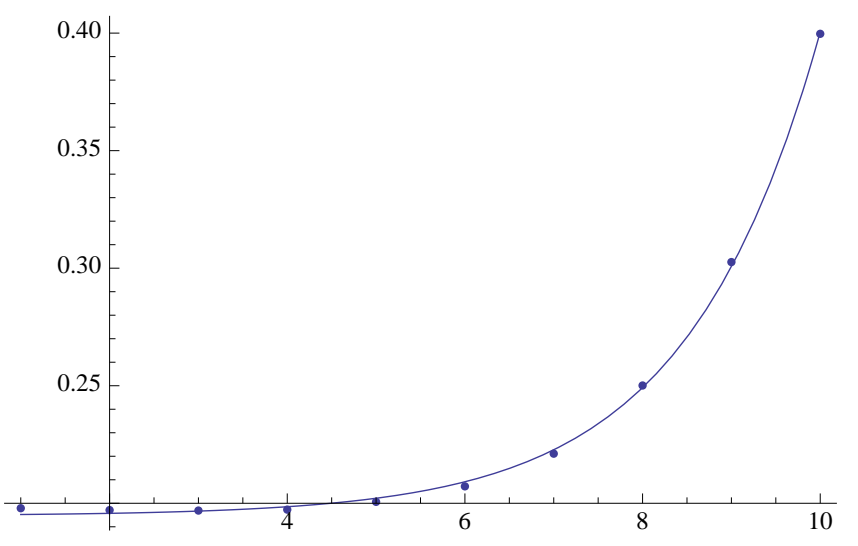

E3exp $=$ NonlinearModelFit $\left[E 3 d a t a[[1 ; ; 28]], b+d 0 * a^{\wedge} \mathbf{x},\{b, d 0, a\}, \mathbf{x}, \operatorname{MaxIterations} \rightarrow 10000\right]$

FittedModel $\left[0.188313+4.72056 \times 10^{-8} \ll 18 \gg^{x}\right]$

E3exp ["BestFit"]

$0.188313+4.72056 \times 10^{-8} 1.72559^{x}$

Show [ListPlot [E3data $[[1 ; ; 28]], \mathrm{PlotRange} \rightarrow \mathrm{All}], \mathrm{Plot}[\mathrm{E} 3 \exp [\mathbf{x}],\{\mathbf{x}, 1,31\}]]$

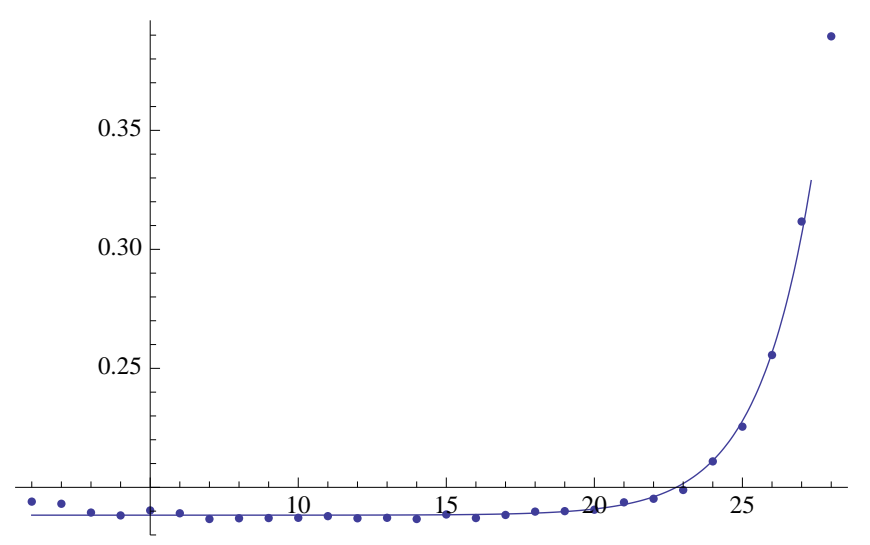

E4exp $=$ NonlinearModelFit $\left[E 4\right.$ data $[[1 ; ; 24]], b+d 0 * a^{\wedge} \mathbf{x},\{b, d 0, a\}, \mathbf{x}$, MaxIterations $\left.\rightarrow 10000\right]$

FittedModel $\left[0.196468+1.87325 \times 10^{-7} \ll 18 \gg^{x}\right]$

E4exp ["BestFit"]

$0.196468+1.87325 \times 10^{-7} 1.79141^{x}$ 
Show [ListPlot $[E 4$ data $[[1 ; ; 24]], P l o t R a n g e \rightarrow A l l], P l o t[E 4 \exp [\mathbf{x}],\{x, 1,28\}]]$

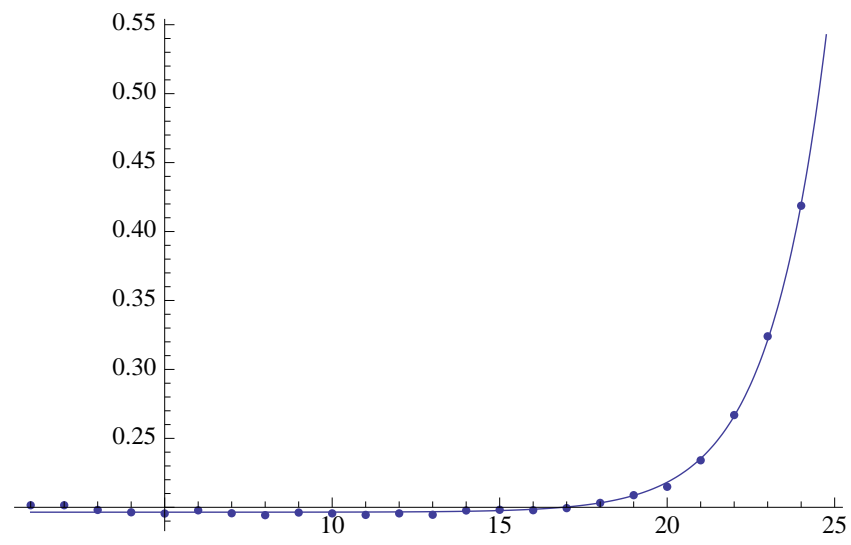

E5exp $=$ NonlinearModelFit $\left[E 5 d a t a[[1 ; ; 20]], b+d 0 * a^{\wedge} \mathbf{x},\{b, d 0, a\}, \mathbf{x}\right.$, MaxIterations $\left.\rightarrow 10000\right]$ FittedModel $\left[0.195601+1.61624 \times 10^{-6} \ll 19 \gg^{x}\right]$

E5exp ["BestFit"]

$0.195601+1.61624 \times 10^{-6} 1.79682^{x}$

Show $[$ ListPlot $[\mathbf{E} 5$ data $[[1 ; ; 20]], P l o t R a n g e \rightarrow A l l], P l o t[E 5 \exp [\mathbf{x}],\{\mathbf{x}, 1,23\}]]$

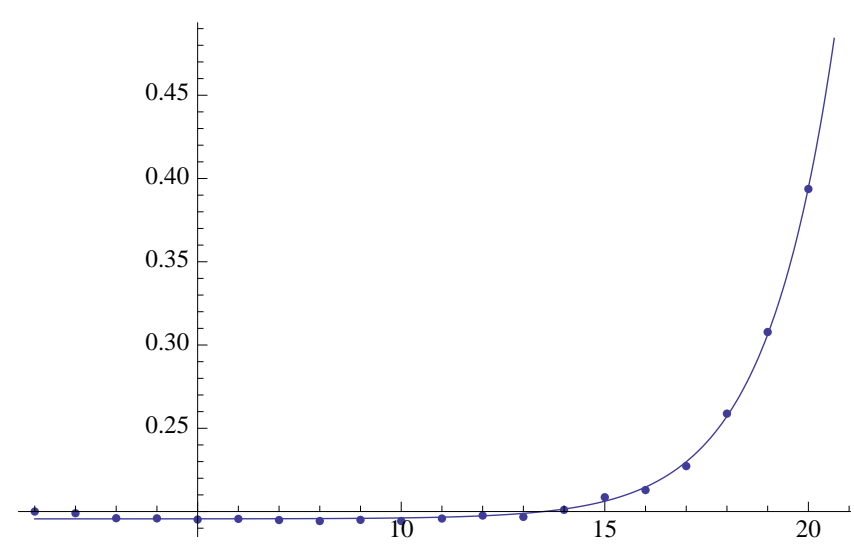

E6exp $=$ NonlinearModelFit $\left[\mathbf{E} 6\right.$ data $\left.[[1 ; ; 17]], b+d 0 * a^{\wedge} \mathbf{x},\{b, d 0, a\}, \mathbf{x}, \operatorname{MaxIterations} \rightarrow 10000\right]$ FittedModel $\left[0.191688+5.26034 \times 10^{-6} \ll 18 \gg^{x}\right]$

E6exp ["BestFit"]

$0.191688+5.26034 \times 10^{-6} 1.8553^{x}$ 
Show [ListPlot $[\mathrm{E} 6$ data $[1 ; ; 17]], \operatorname{PlotRange} \rightarrow \mathrm{All}], \mathrm{Plot}[\mathrm{E} 6 \exp [\mathbf{x}],\{\mathbf{x}, 1,19\}]]$

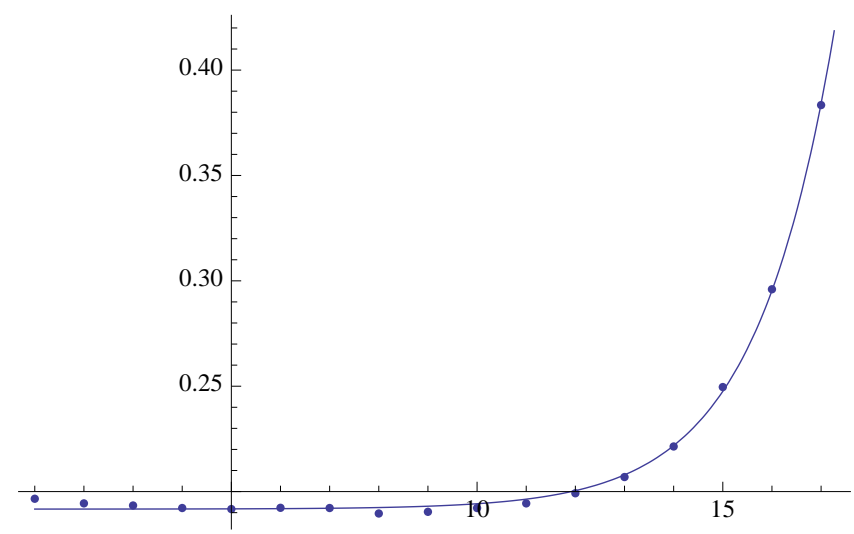

E7exp $=$ NonlinearModelFit $\left[E 7 d a t a[[1 ; ; 13]], b+d 0 * a^{\wedge} \mathbf{x},\{b, d 0, a\}, \mathbf{x}, \operatorname{MaxIterations} \rightarrow 10000\right]$ FittedModel $\left[0.190447+0.0000264797 \ll 19 \gg^{x}\right]$

E7exp ["BestFit"]

$0.190447+0.00002647971 .94885^{x}$

Show [ListPlot $[E 7$ data $[1 ; ; 13]]$, PlotRange $\rightarrow A l 1], \operatorname{Plot}[\mathbf{E} 7 \exp [\mathbf{x}],\{\mathbf{x}, 1,15\}]]$

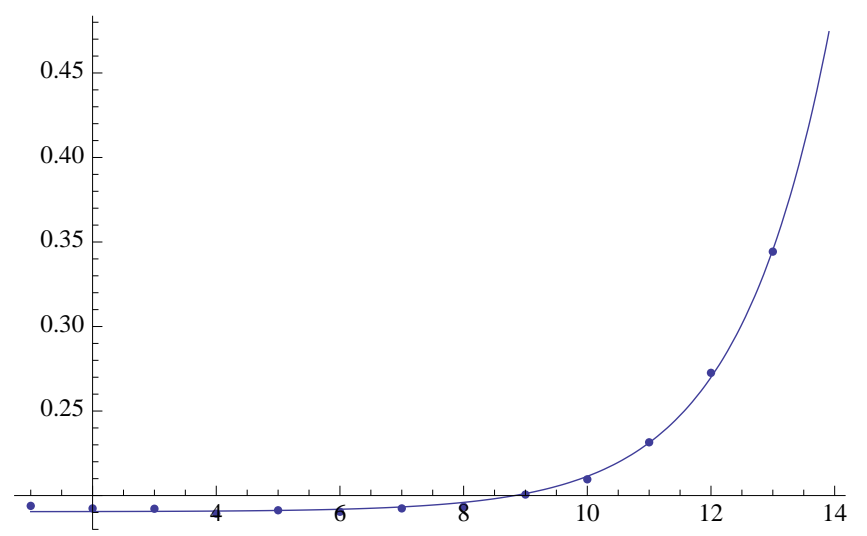

E8exp $=$ NonlinearModelFit $\left[E 8 d a t a[[1 ; ; 10]], b+d 0 * a^{\wedge} \mathbf{x},\{b, d 0, a\}, \mathbf{x}\right.$, MaxIterations $\left.\rightarrow 10000\right]$ FittedModel $\left[0.200804+0.000345749 \ll 18 \gg^{x}\right]$

E8exp [ BestFit"]

$0.200804+0.0003457491 .91906^{x}$ 
Show [ListPlot $[E 8 d a t a[[1 ; 10]], \operatorname{PlotRange~} \rightarrow A l 1], \operatorname{Plot}[\mathbf{E 8 e x p}[\mathbf{x}],\{\mathbf{x}, 1,10\}]]$
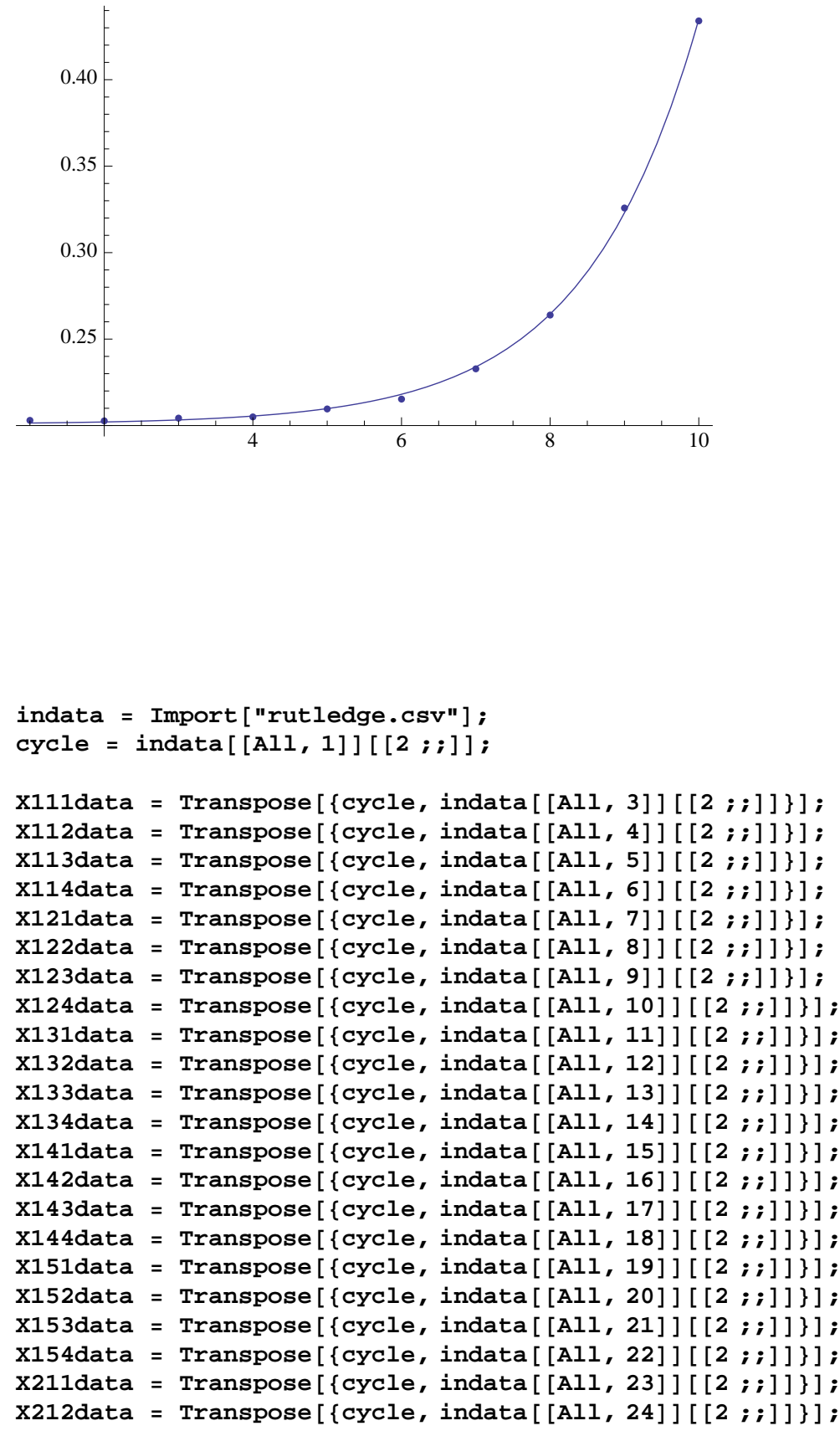


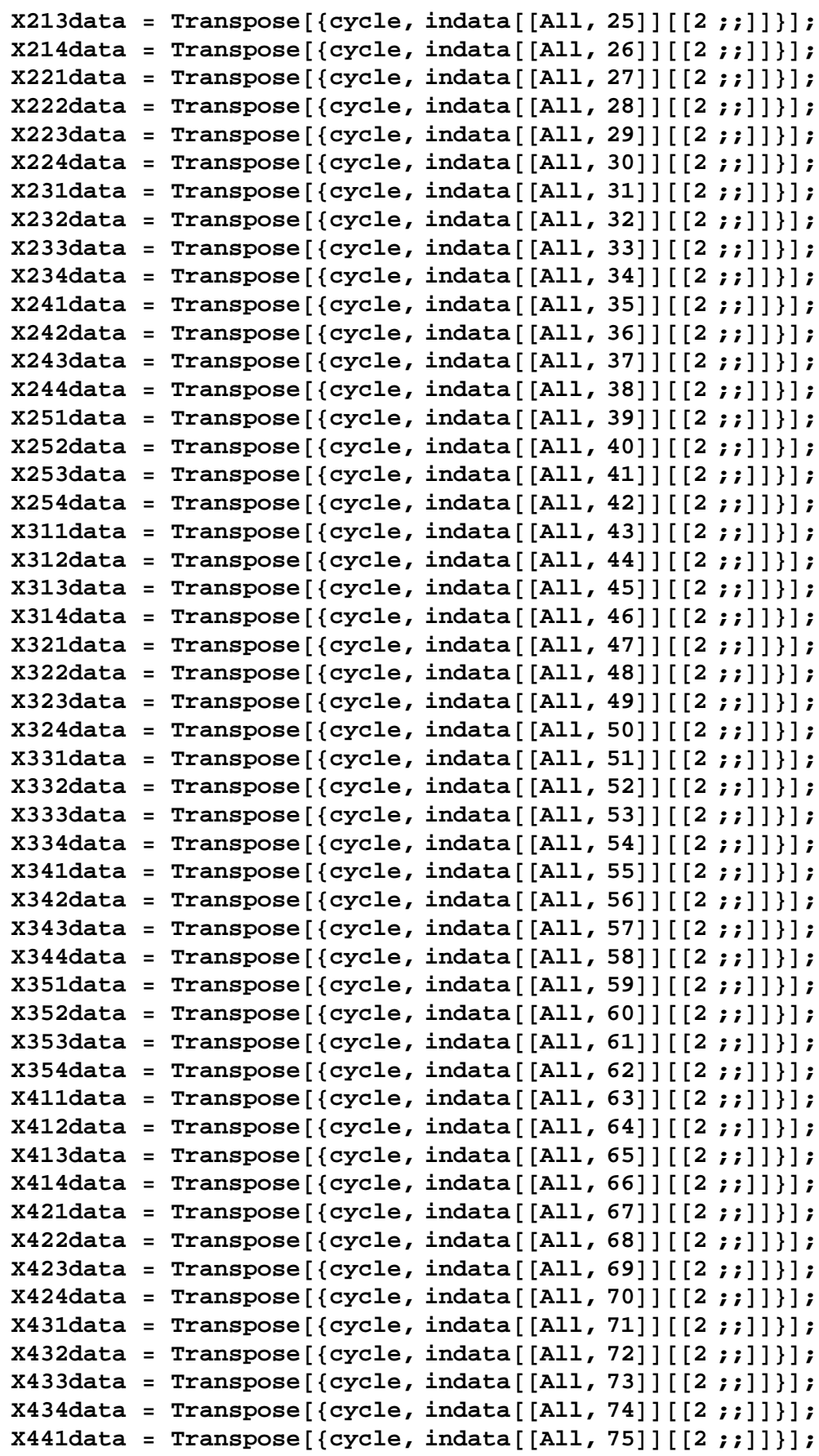




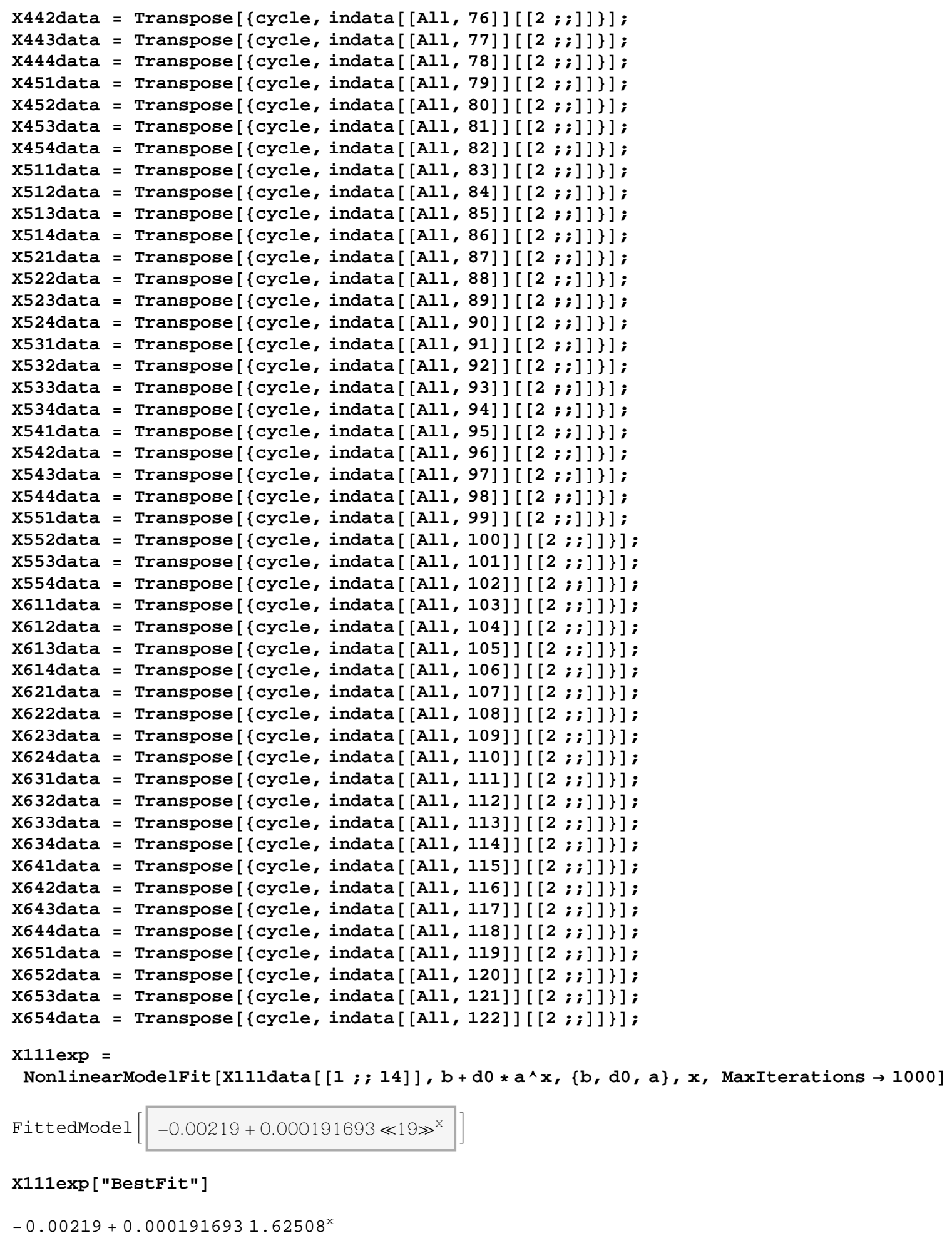




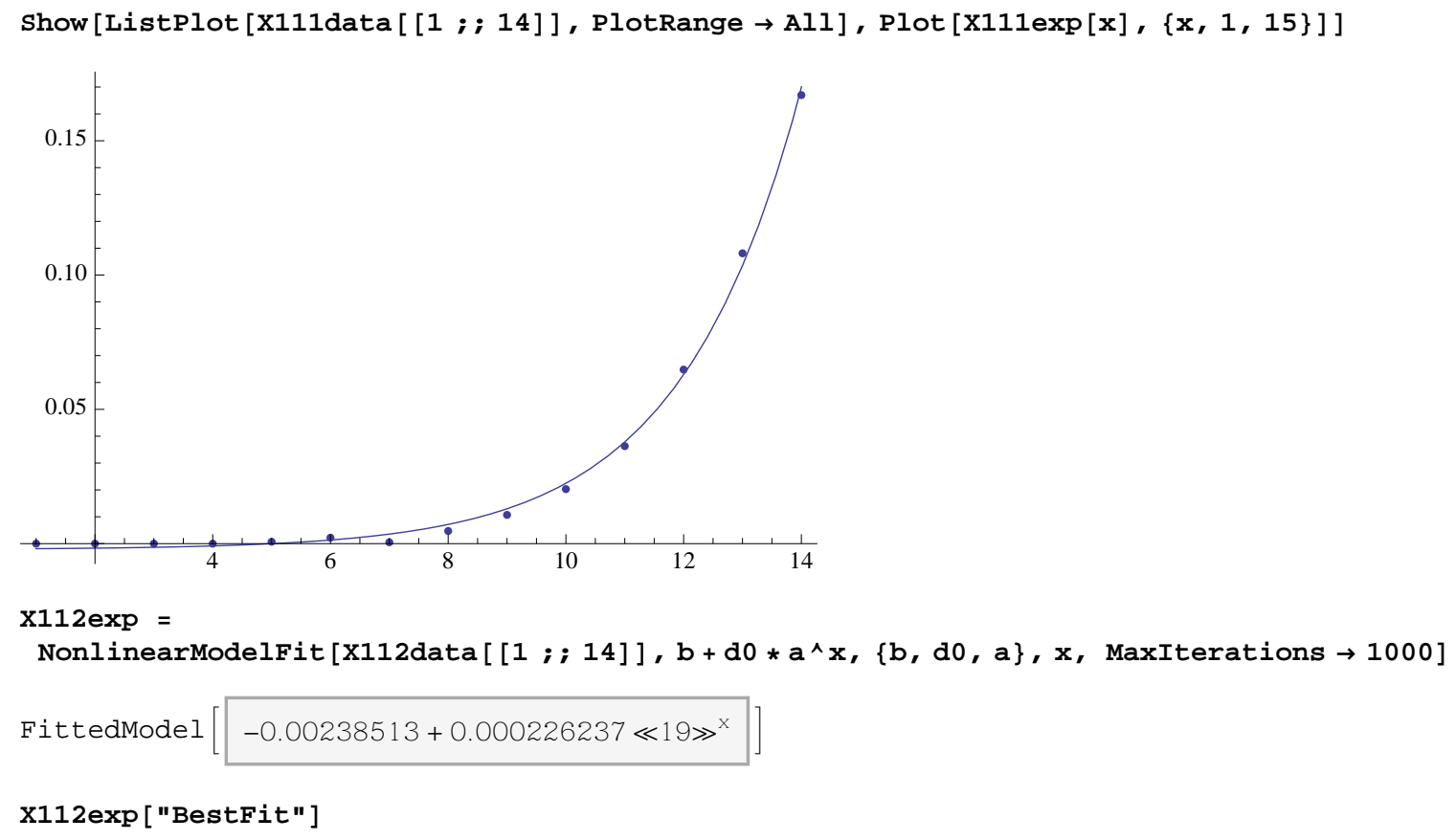

$-0.00238513+0.0002262371 .62638^{x}$

Show [ListPlot $[\mathrm{X} 112 \operatorname{dat}[[1 ; ; 14]], \mathrm{PlotRange} \rightarrow \mathrm{All}], \mathrm{Plot}[\mathrm{X} 112 \exp [\mathbf{x}],\{\mathbf{x}, 1,15\}]]$

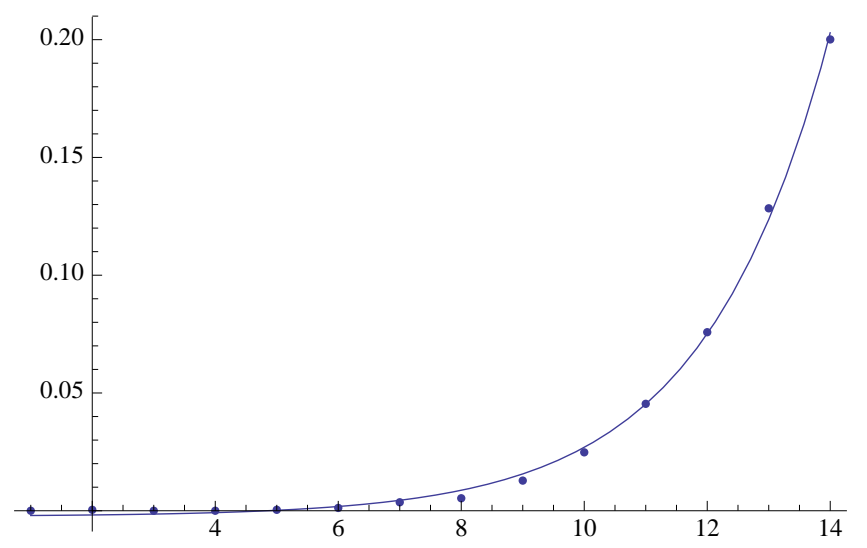

$\mathrm{x} 113 \exp =$

NonlinearModelFit $[\mathrm{X} 113 \mathrm{dat} a[1 ; ; 14]], \mathrm{b}+\mathrm{d} 0 * \mathrm{a}^{\wedge} \mathbf{x},\{\mathrm{b}, \mathrm{d0}, \mathrm{a}\}, \mathbf{x}, \operatorname{Max}$ Iterations $\left.\rightarrow 1000\right]$

FittedModel $\left[-0.00191785+0.000166168 \ll 18 \gg^{x}\right]$

X113exp ["BestFit"]

$-0.00191785+0.0001661681 .6501^{x}$ 
Show $[$ ListPlot $[\mathrm{X} 113 \operatorname{dat}[[1 ; ; 14]], \mathrm{PlotRange} \rightarrow \mathrm{All}], \mathrm{Plot}[\mathrm{X} 113 \exp [\mathbf{x}],\{\mathbf{x}, 1,15\}]]$

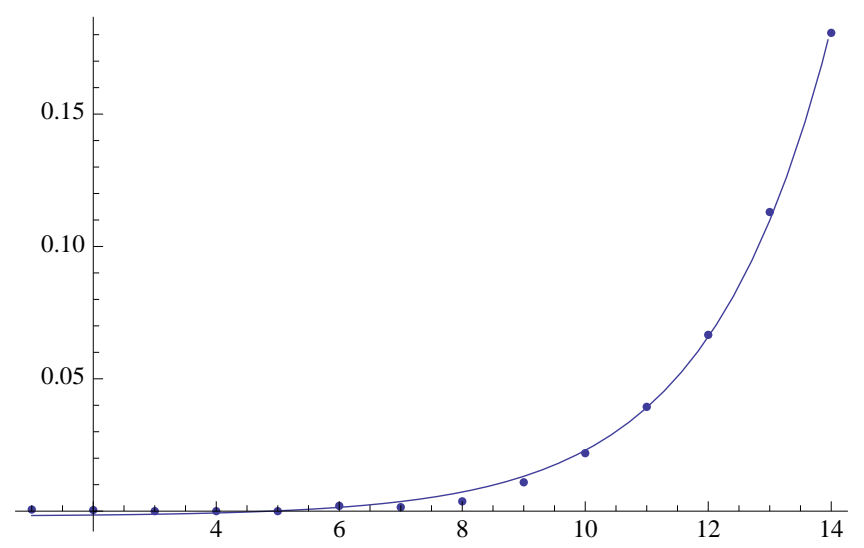

$\mathrm{x} 114 \mathrm{exp}=$

NonlinearModelFit $\left[\mathrm{X} 114\right.$ data $[[1 ; ; 14]], b+d 0 * a^{\wedge} \mathbf{x},\{b, d 0, a\}, \mathbf{x}$, MaxIterations $\left.\rightarrow 1000\right]$

FittedModel $\left[-0.0020944+0.000187687 \ll 19 \gg^{x}\right]$

X114exp ["BestFit"]

$-0.0020944+0.0001876871 .63819^{x}$

Show [ListPlot $[\mathrm{X} 114$ data $[1 ; ; 14]], \mathrm{PlotRange} \rightarrow \mathrm{All}], \mathrm{Plot}[\mathrm{X} 114 \exp [\mathbf{x}],\{\mathbf{x}, 1,15\}]]$

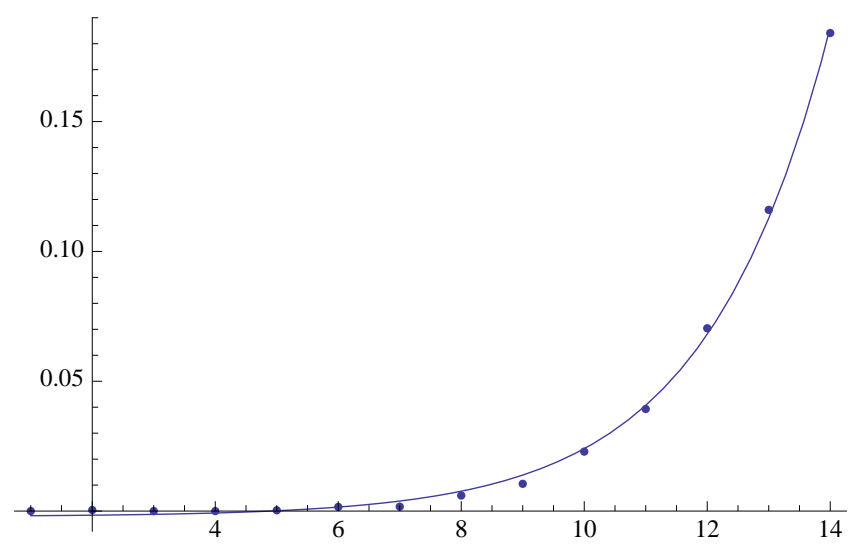

$\mathrm{x} 121 \mathrm{exp}=$

NonlinearModelFit $\left[\mathrm{X} 121\right.$ data $[[1 ; ; 14]], b+d 0 * a^{\wedge} \mathbf{x},\{b, d 0, a\}, \mathbf{x}$, MaxIterations $\left.\rightarrow 1000\right]$

FittedModel $\left[-0.00161267+0.000160324 \ll 19 \gg^{x}\right]$

x121exp ["BestFit"]

$-0.00161267+0.0001603241 .62227^{x}$ 
Show $[$ ListPlot $[\mathrm{X} 121$ data $[[1 ; 114]], \mathrm{PlotRange} \rightarrow \mathrm{All}], \mathrm{Plot}[\mathrm{X} 121 \exp [\mathbf{x}],\{\mathrm{x}, 1,15\}]]$

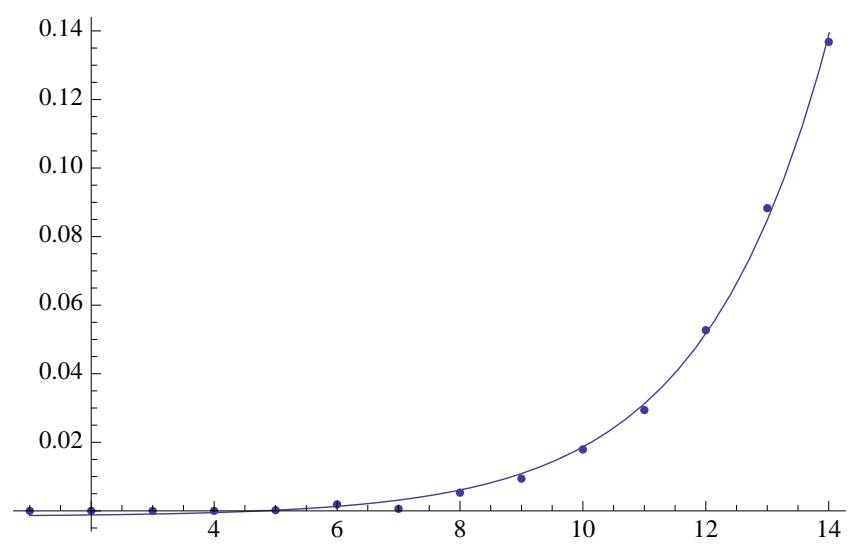

$\mathrm{x} 122 \mathrm{exp}=$

NonlinearModelFit $\left[\mathrm{X} 122\right.$ data $[[1 ; ; 14]], b+d 0 * a^{\wedge} \mathbf{x},\{b, d 0, a\}, \mathbf{x}$, MaxIterations $\left.\rightarrow 1000\right]$

FittedModel $\left[-0.00145194+0.000118936 \ll 19 \gg^{x}\right]$

X122exp ["BestFit"]

$-0.00145194+0.0001189361 .66126^{x}$

Show [ListPlot $[\mathrm{X} 122 \operatorname{dat}[[1 ; ; 14]], \mathrm{PlotRange} \rightarrow \mathrm{All}], \mathrm{Plot}[\mathrm{X} 122 \exp [\mathbf{x}],\{\mathbf{x}, 1,15\}]]$

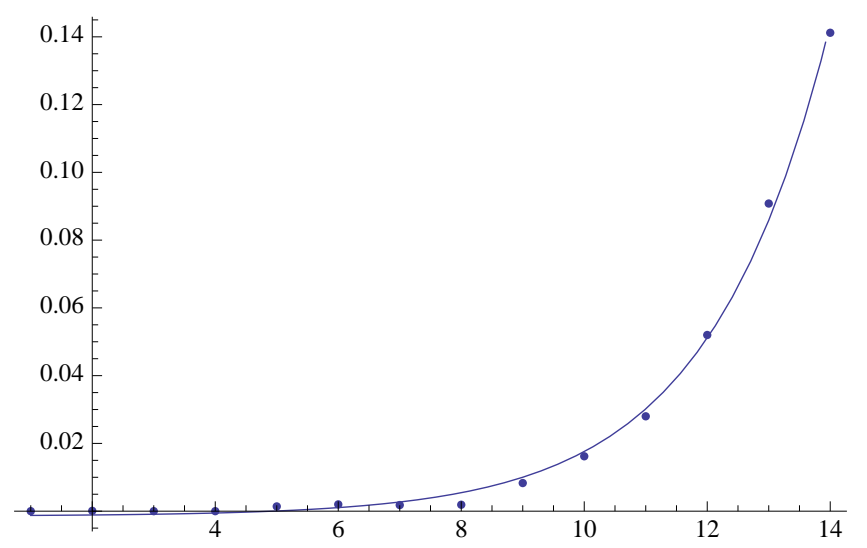

$\mathrm{x} 123 \mathrm{exp}=$

NonlinearModelFit $\left[\mathrm{X} 123\right.$ data $[[2 ; ; 14]], b+d 0 * a^{\wedge} \mathbf{x},\{b, d 0, a\}, \mathbf{x}$, MaxIterations $\left.\rightarrow 1000\right]$

FittedModel $\left[-0.00159448+0.000156214 \ll 18 \gg^{x}\right]$

x123exp ["BestFit"]

$-0.00159448+0.0001562141 .6133^{x}$ 
Show [ListPlot $[\mathrm{X} 123 \operatorname{dat}[[1 ; ; 14]], \mathrm{PlotRange} \rightarrow \mathrm{All}], \mathrm{Plot}[\mathrm{X} 123 \exp [\mathbf{x}],\{\mathbf{x}, 1,15\}]]$

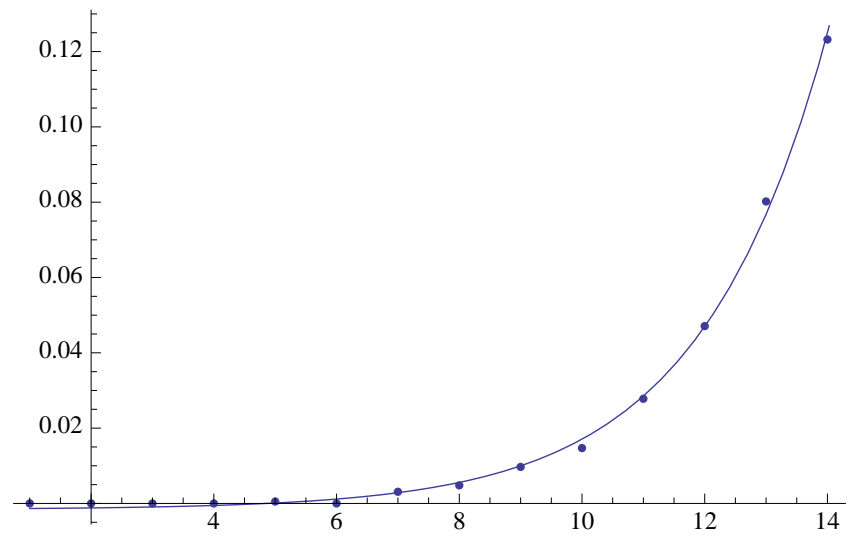

$\mathrm{X} 124 \mathrm{exp}=$

NonlinearModelFit $\left[\mathrm{X} 124\right.$ data $[[2 ; ; 14]], b+d 0 * a^{\wedge} \mathbf{x},\{b, d 0, a\}, \mathbf{x}, \operatorname{Max}$ Iterations $\left.\rightarrow 1000\right]$

FittedModel $\left[-0.00133576+0.000125712 \ll 19 \gg^{x}\right]$

x124exp ["BestFit"]

$-0.00133576+0.0001257121 .63696^{x}$

Show [ListPlot $[\mathrm{X} 124$ data $[1 ; ; 14]], \mathrm{PlotRange} \rightarrow \mathrm{All}], \mathrm{Plot}[\mathrm{X} 124 \exp [\mathbf{x}],\{\mathbf{x}, 1,15\}]]$

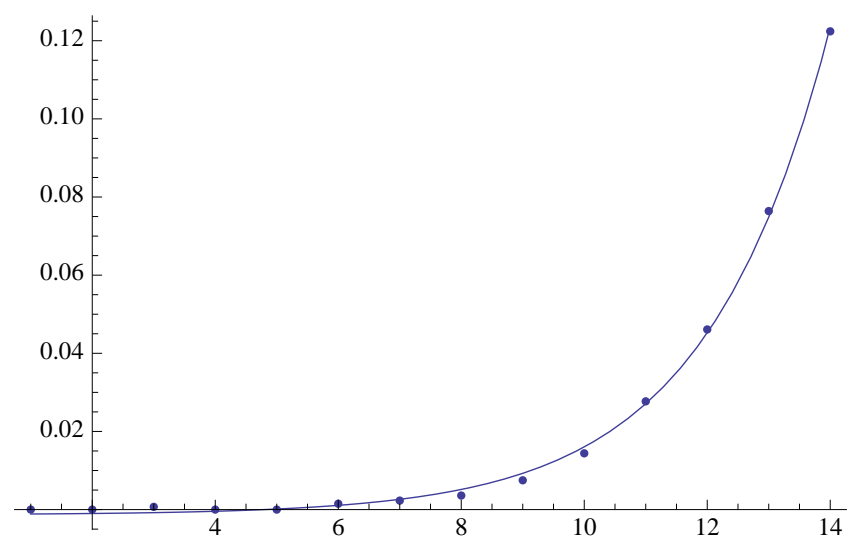

$\mathrm{x} 131 \mathrm{exp}=$

NonlinearModelFit $\left[\mathrm{X} 131\right.$ data $[[2 ; ; 14]], b+d 0 * a^{\wedge} \mathbf{x},\{b, d 0, a\}, \mathbf{x}$, MaxIterations $\left.\rightarrow 1000\right]$

FittedModel $\left[-0.00100539+0.000137609 \ll 18 \gg^{x}\right]$

x131exp ["BestFit"]

$-0.00100539+0.0001376091 .61801^{x}$ 


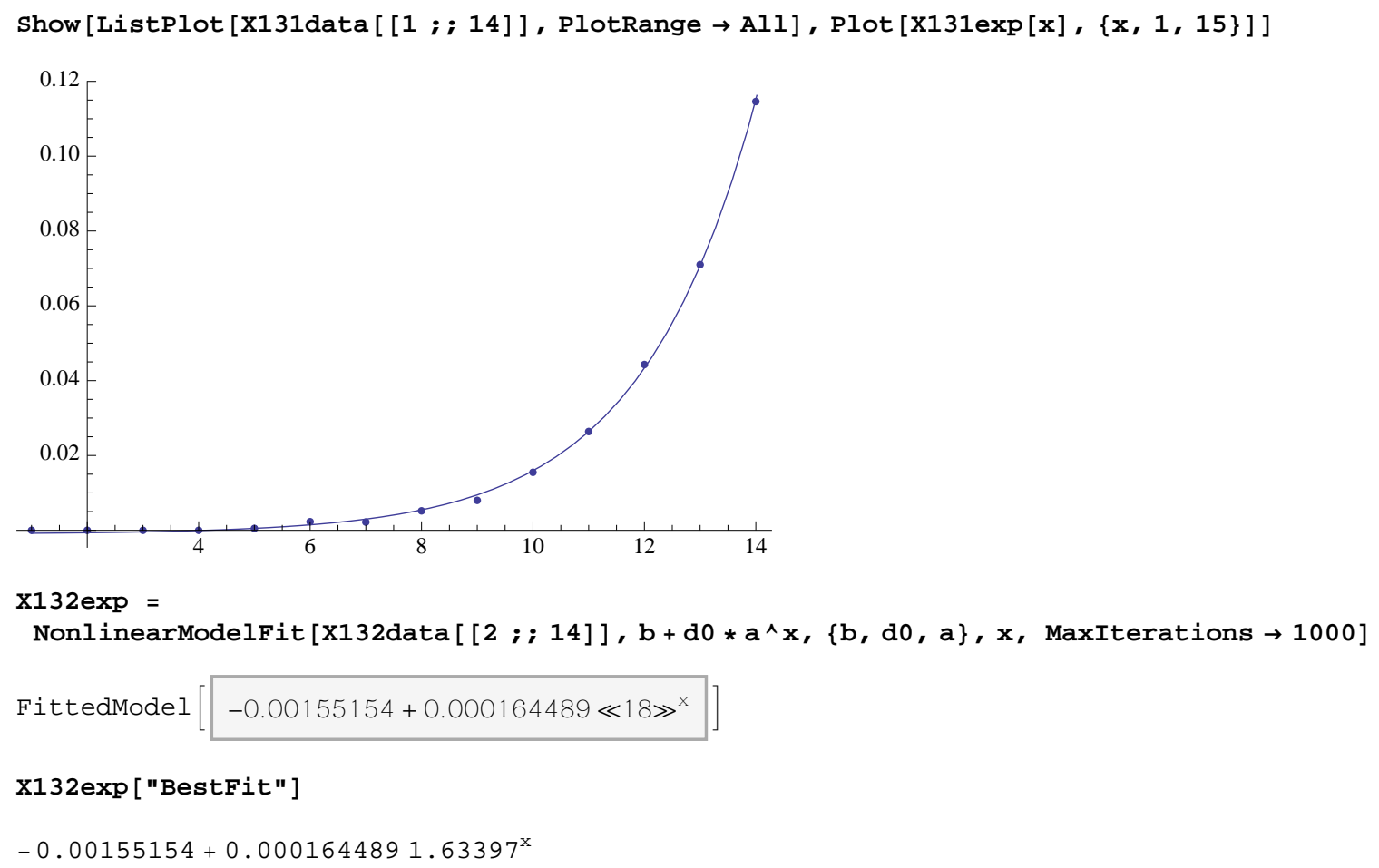

Show $[$ ListPlot $[\mathrm{X} 132 \operatorname{data}[[1 ; ; 14]], \mathrm{PlotRange} \rightarrow \mathrm{All}], \mathrm{Plot}[\mathrm{X} 132 \exp [\mathbf{x}],\{\mathbf{x}, 1,15\}]]$

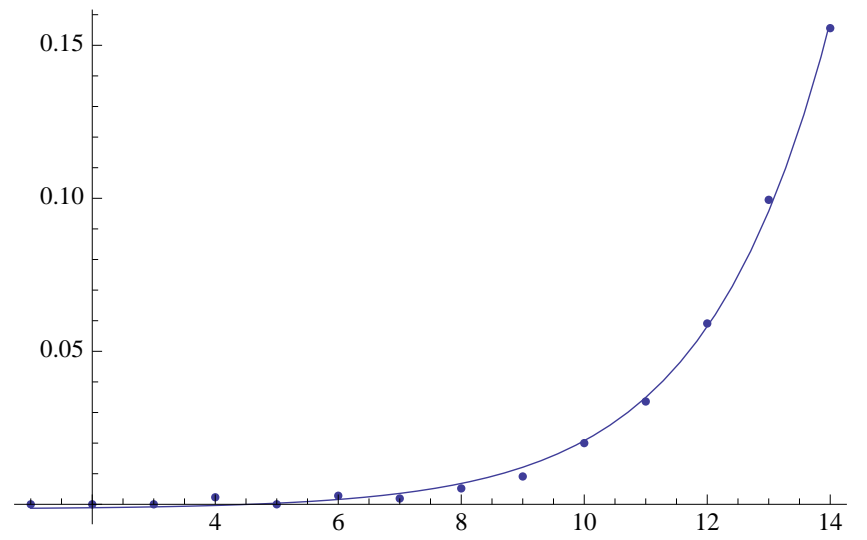

$\mathrm{x} 133 \mathrm{exp}=$

NonlinearModelFit $[\mathbf{x} 133$ data $[[2 ; ; 14]], b+d 0 * a \wedge \mathbf{x},\{b, d 0, a\}, \mathbf{x}, \operatorname{MaxIterations} \rightarrow 1000]$

FittedModel $\left[-0.00176055+0.000176786 \ll 19 \gg^{x}\right]$

$\mathrm{X} 133 \exp [$ "BestFit"]

$-0.00176055+0.0001767861 .61879^{x}$ 
Show [ListPlot $[\mathrm{X} 133 \operatorname{dat}[[1 ; ; 14]], \mathrm{PlotRange} \rightarrow \mathrm{All}], \mathrm{Plot}[\mathrm{X} 133 \exp [\mathbf{x}],\{\mathbf{x}, 1,15\}]]$

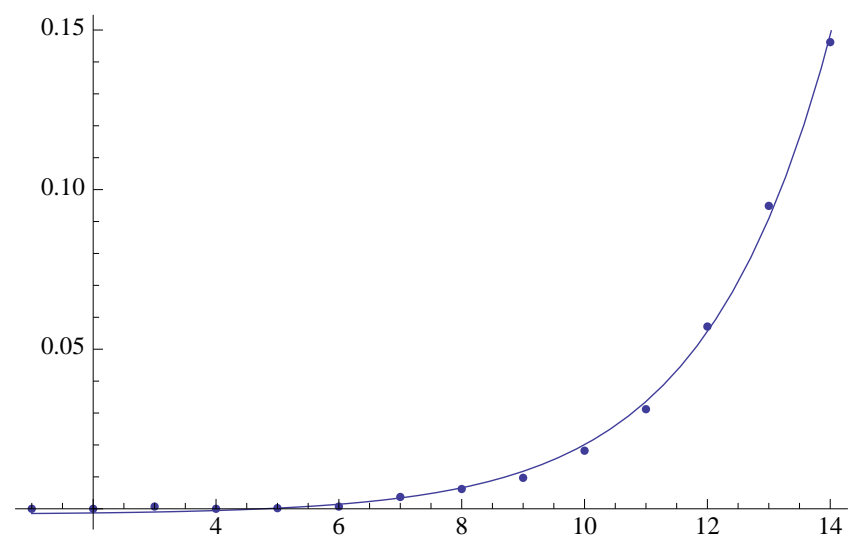

$\mathrm{x} 134 \mathrm{exp}=$

NonlinearModelFit $\left[\mathrm{x} 134\right.$ data $\left.[[2 ; ; 14]], b+d 0 * a^{\wedge} \mathbf{x},\{b, d 0, a\}, \mathbf{x}, \operatorname{MaxIterations} \rightarrow 1000\right]$

FittedModel $\left[-0.00103387+0.000145468 \ll 19 \gg^{x}\right]$

x134exp ["BestFit"]

$-0.00103387+0.0001454681 .62944^{x}$

Show [ListPlot $[\mathrm{X} 134$ data $[1 ; ; 14]], \mathrm{PlotRange} \rightarrow \mathrm{All}], \mathrm{Plot}[\mathrm{X} 134 \exp [\mathbf{x}],\{\mathbf{x}, 1,15\}]]$

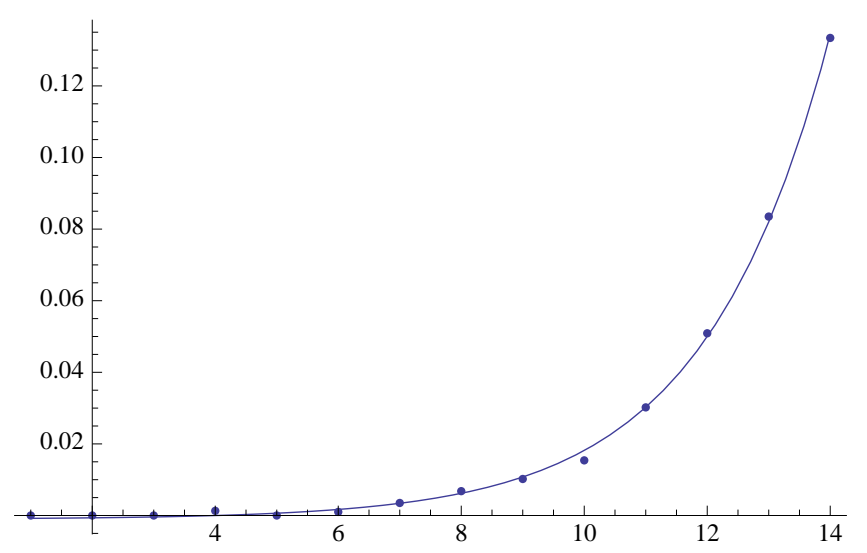

$\mathrm{x} 141 \mathrm{exp}=$

NonlinearModelFit $[\mathrm{x} 141$ data $\left.[2 ; ; 14]], b+d 0 * a^{\wedge} \mathbf{x},\{b, d 0, a\}, \mathbf{x}, \operatorname{MaxIterations} \rightarrow 1000\right]$

FittedModel $\left[-0.00144893+0.000119027 \ll 19 \gg^{x}\right]$

x141exp ["BestFit"]

$-0.00144893+0.0001190271 .63747^{x}$ 
Show $[$ ListPlot $[\mathrm{X} 141$ data $[1 ; ; 14]], \mathrm{PlotRange} \rightarrow \mathrm{All}], \mathrm{Plot}[\mathrm{X} 141 \exp [\mathrm{x}],\{\mathrm{x}, 1,15\}]]$

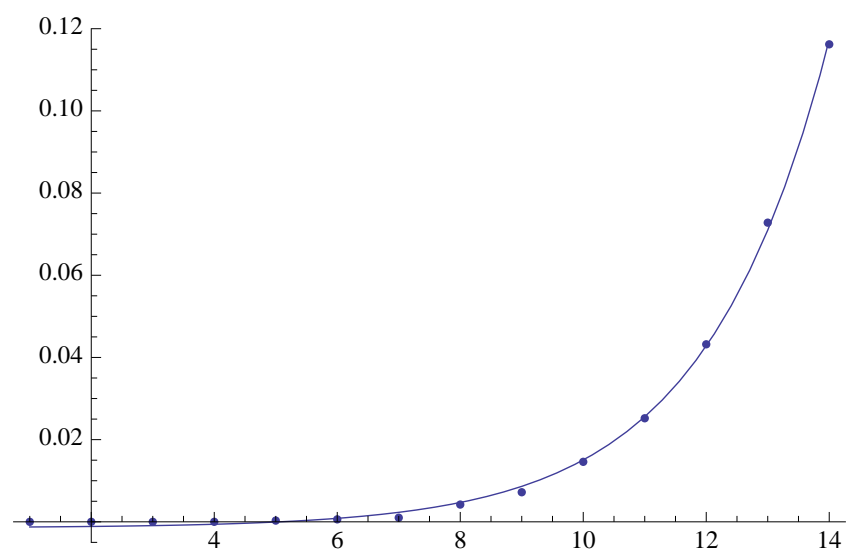

$\mathrm{x} 142 \mathrm{exp}=$

NonlinearModelFit $[\mathrm{x} 142$ data $\left.[2 ; ; 14]], b+d 0 * a^{\wedge} \mathbf{x},\{b, d 0, a\}, \mathbf{x}, \operatorname{MaxIterations} \rightarrow 1000\right]$

FittedModel $\left[-0.00170321+0.000156386 \ll 19 \gg^{x}\right]$

x142exp ["BestFit"]

$-0.00170321+0.0001563861 .62582^{x}$

Show $[$ ListPlot $[\mathrm{X} 142 \operatorname{dat} a[1 ; ; 14]], \mathrm{PlotRange} \rightarrow \mathrm{All}], \mathrm{P} 1$ ot $[\mathrm{X} 142 \exp [\mathrm{x}],\{\mathrm{x}, 1,15\}]]$

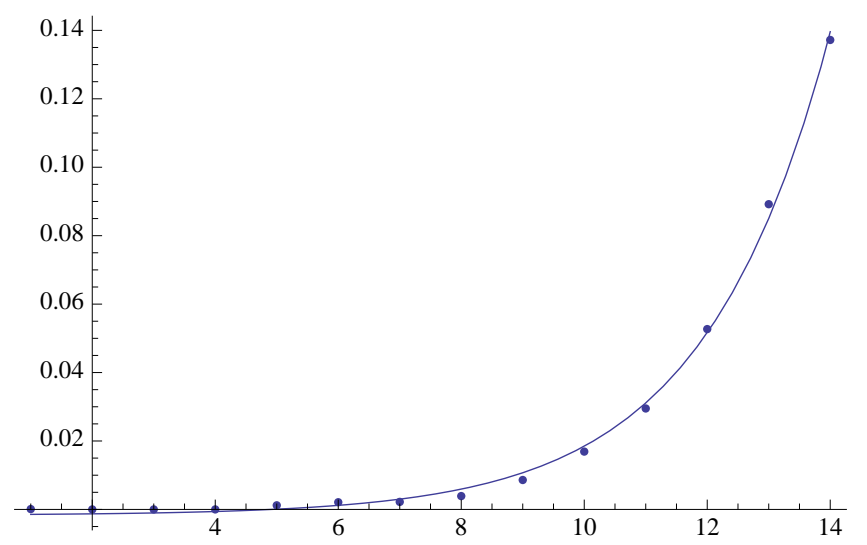

$\mathrm{X} 143 \mathrm{exp}=$

NonlinearModelFit [X143data $[2 ; ; 14]], b+d 0 * a \wedge x,\{b, d 0, a\}, \mathbf{x}$, MaxIterations $\rightarrow 1000]$

FittedModel $\left[-0.00201219+0.000157495 \ll 19 \gg^{x}\right]$

X143exp ["BestFit"]

$-0.00201219+0.0001574951 .61995^{x}$ 
Show $[$ ListPlot $[\mathrm{X} 143 \operatorname{dat}[[1 ; ; 14]], \mathrm{PlotRange} \rightarrow \mathrm{All}], \mathrm{Plot}[\mathrm{X} 143 \exp [\mathrm{x}],\{\mathrm{x}, 1,15\}]]$

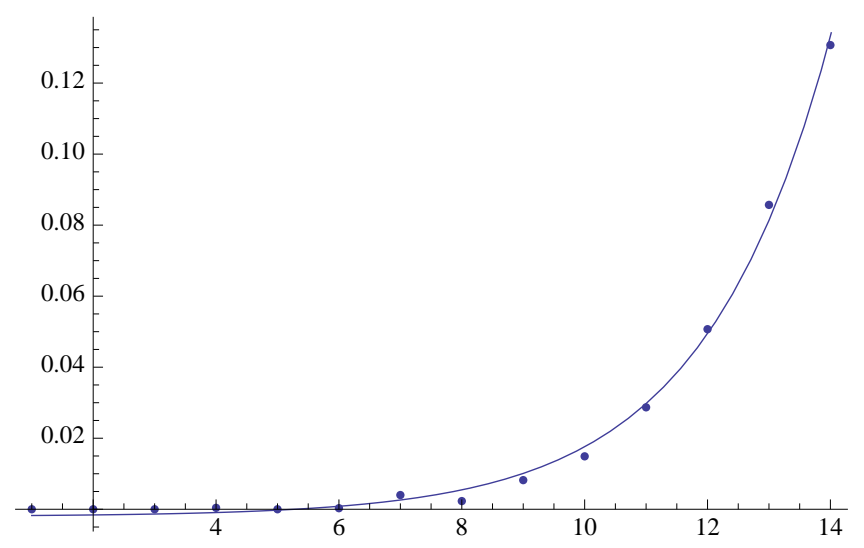

$\mathrm{x} 144 \exp =$

NonlinearModelFit $[\mathrm{x} 144$ data $[2 ; ; 14]], b+d 0 * a^{\wedge} \mathbf{x},\{b, d 0, a\}, \mathbf{x}, \operatorname{Max}$ Iterations $\left.\rightarrow 1000\right]$

FittedModel $\left[-0.00162929+0.000189251 \ll 19 \gg^{x}\right.$

x144exp ["BestFit"]

$-0.00162929+0.0001892511 .60171^{x}$

Show [ListPlot $[\mathrm{X} 144$ data $[[1 ; 114]], \mathrm{PlotRange} \rightarrow \mathrm{All}], \mathrm{Plot}[\mathrm{X} 144 \exp [\mathrm{x}],\{\mathrm{x}, 1,15\}]]$

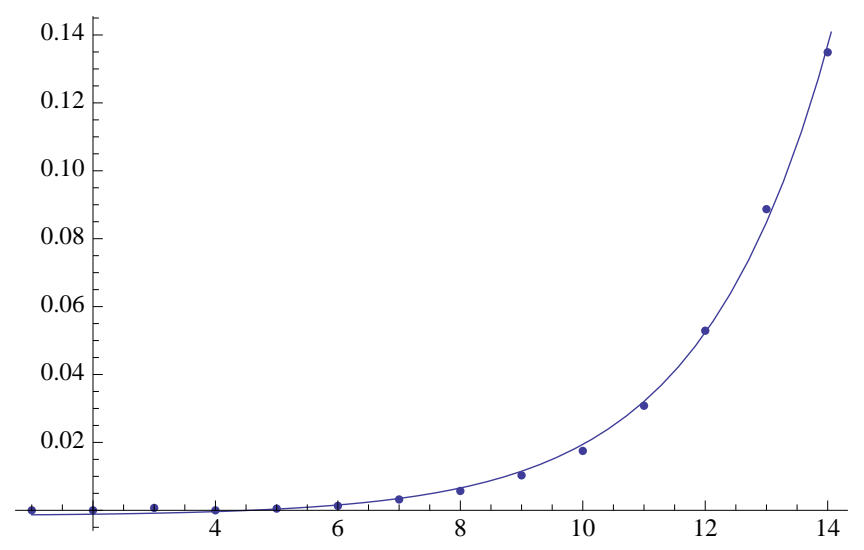

$\mathrm{x} 151 \mathrm{exp}=$

NonlinearModelFit $\left[\mathrm{x} 151\right.$ data $\left.[[2 ; ; 14]], b+d 0 * a^{\wedge} \mathbf{x},\{b, d 0, a\}, \mathbf{x}, \operatorname{MaxIterations} \rightarrow 1000\right]$

FittedModel $\left[-0.00233291+0.000168334 \ll 19 \gg^{x}\right]$

x151exp ["BestFit"]

$-0.00233291+0.0001683341 .62716^{x}$ 
Show $[$ ListPlot $[\mathrm{X} 151$ data $[[1 ; 14]], \mathrm{PlotRange} \rightarrow \mathrm{All}], \mathrm{Plot}[\mathrm{X} 151 \exp [\mathrm{x}],\{\mathrm{x}, 1,15\}]]$

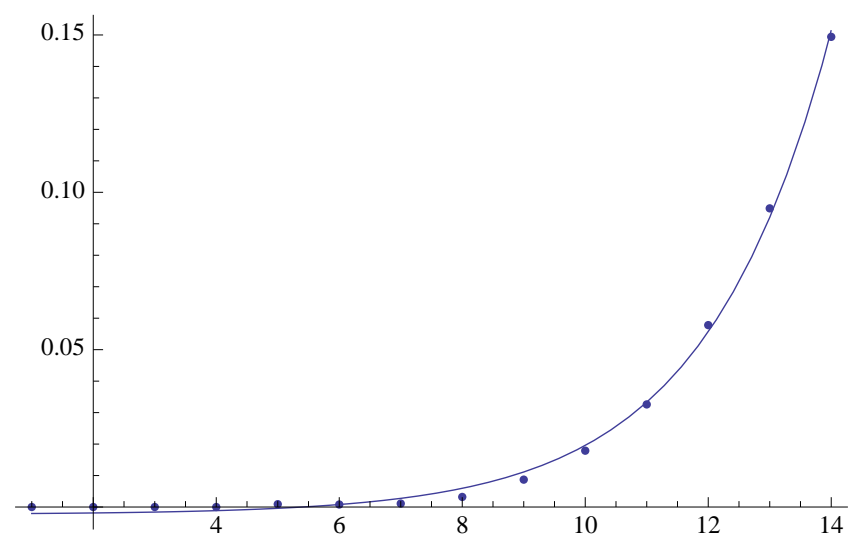

$\mathrm{x} 152 \mathrm{exp}=$

NonlinearModelFit $\left[\mathrm{X} 152\right.$ data $[[2 ; ; 14]], b+d 0 * a^{\wedge} \mathbf{x},\{b, d 0, a\}, \mathbf{x}, \operatorname{Max}$ Iterations $\left.\rightarrow 1000\right]$

FittedModel $\left[-0.0021698+0.000170541 \ll 19 \gg^{x}\right.$

x152exp ["BestFit"]

$-0.0021698+0.0001705411 .6316^{x}$

Show $[$ ListPlot $[\mathrm{X} 152$ data $[[1 ; 114]], \mathrm{PlotRange} \rightarrow \mathrm{All}], \mathrm{Plot}[\mathrm{X} 152 \exp [\mathbf{x}],\{\mathbf{x}, 1,15\}]]$

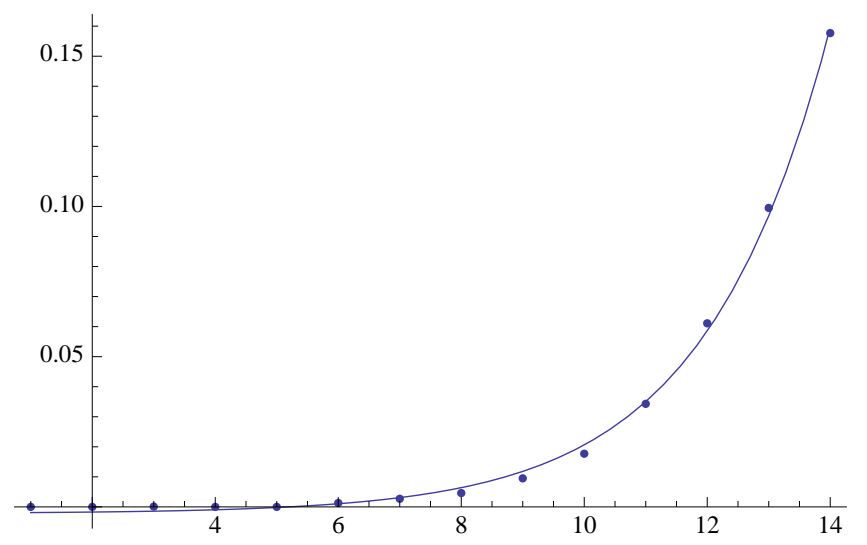

$\mathrm{x} 153 \mathrm{exp}=$

NonlinearModelFit $[\mathbf{x} 153$ data $\left.[2 ; ; 14]], b+d 0 * a^{\wedge} \mathbf{x},\{b, d 0, a\}, \mathbf{x}, \operatorname{MaxIterations} \rightarrow 1000\right]$

FittedModel $\left[-0.00209415+0.000197078 \ll 19 \gg^{x}\right]$

X153exp ["BestFit"]

$-0.00209415+0.0001970781 .61031^{x}$ 
Show [ListPlot $[\mathrm{X} 153$ data $[1 ; ; 14]], \mathrm{PlotRange} \rightarrow \mathrm{All}], \mathrm{Plot}[\mathrm{X} 153 \exp [\mathbf{x}],\{\mathbf{x}, 1,15\}]]$

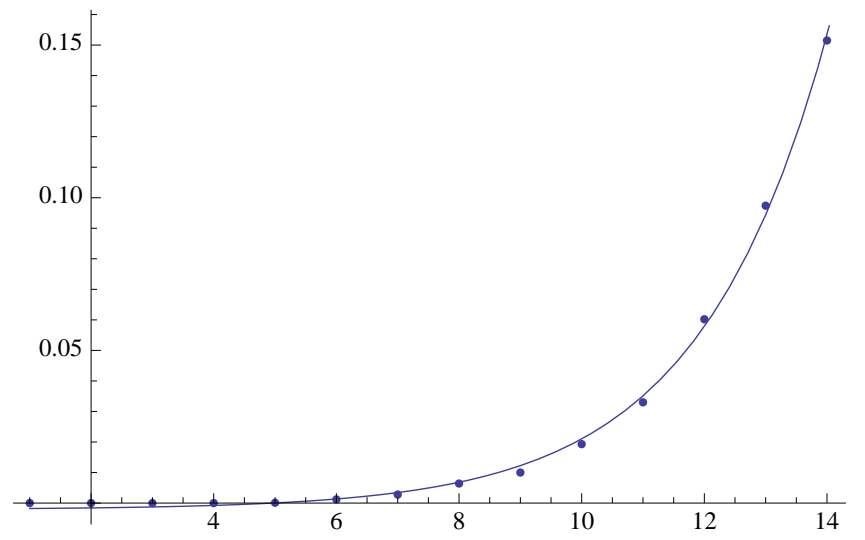

$\mathrm{x} 154 \mathrm{exp}=$

NonlinearModelFit $\left[\mathrm{X} 154\right.$ data $[[2 ; ; 14]], \mathrm{b}+\mathrm{d} 0 * \mathrm{a}^{\wedge} \mathbf{x},\{\mathrm{b}, \mathrm{d0}, \mathrm{a}\}, \mathbf{x}, \operatorname{Max}$ Iterations $\left.\rightarrow 1000\right]$

FittedModel $\left[-0.00176367+0.000138434 \ll 19 \gg^{x}\right]$

X154exp ["BestFit"]

$-0.00176367+0.0001384341 .64197^{x}$

Show [ListPlot $[\mathrm{X} 154$ data $[[1 ; 114]], \mathrm{PlotRange} \rightarrow \mathrm{All}], \mathrm{Plot}[\mathrm{X} 154 \exp [\mathrm{x}],\{\mathrm{x}, 1,15\}]]$

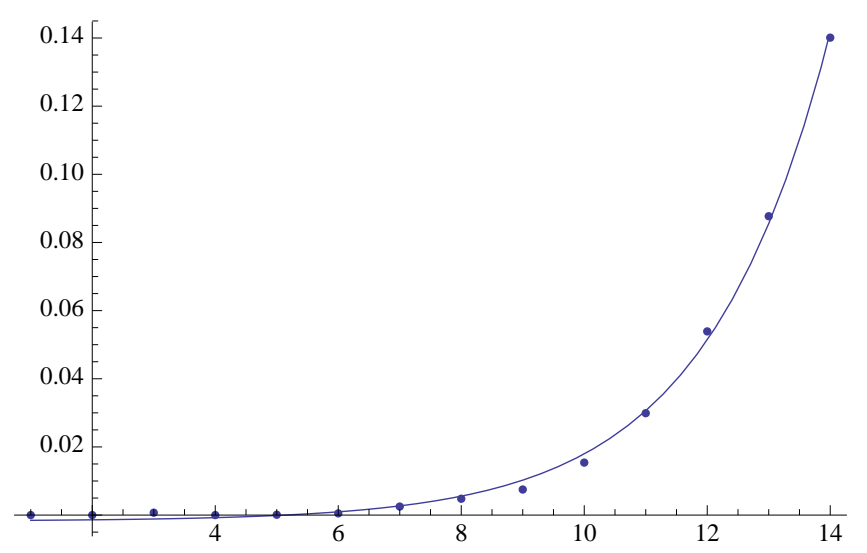

$\mathrm{x} 211 \mathrm{exp}=$

NonlinearModelFit $[\mathbf{x} 211$ data $[1 ; ; 15]], b+d 0 * a^{\wedge} \mathbf{x},\{b, d 0, a\}, \mathbf{x}$, MaxIterations $\left.\rightarrow 1000\right]$

FittedModel $\left[0.000110522+6.11733 \times 10^{-6} \ll 19 \gg^{x}\right]$

X211exp ["BestFit"]

$0.000110522+6.11733 \times 10^{-6} 1.8182^{x}$ 
Show [ListPlot $[\mathrm{X} 211$ data $[[1 ; ; 20]], \mathrm{PlotRange} \rightarrow \mathrm{All}], \mathrm{P} 1$ ot $[\mathrm{X} 211 \exp [\mathbf{x}],\{\mathbf{x}, 1,20\}]]$

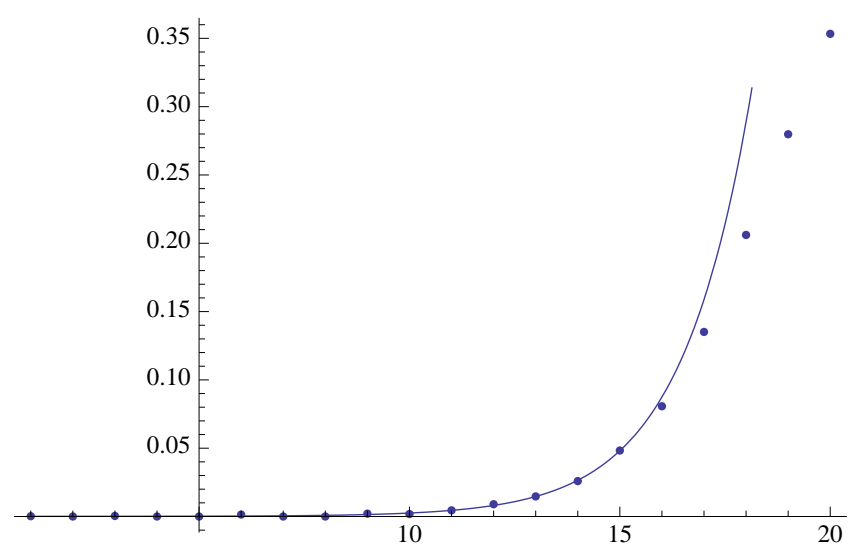

$\mathrm{x} 212 \mathrm{exp}=$

NonlinearModelFit [x212data $[1 ; ; ; 15]], b+d 0 * a \wedge x,\{b, d 0, a\}, \mathbf{x}$, MaxIterations $\rightarrow 1000]$

FittedModel $\left[0.000063897+2.87221 \times 10^{-6} \ll 19 \gg^{x}\right]$

$\mathrm{x} 212 \exp [$ "BestFit"]

$0.000063897+2.87221 \times 10^{-6} 1.90837^{x}$

Show [ListPlot $[\mathrm{X} 212 \operatorname{dat}[[1 ; ; 15]], \mathrm{PlotRange} \rightarrow \mathrm{All}], \mathrm{P} 1$ ot $[\mathrm{X} 212 \exp [\mathbf{x}],\{\mathbf{x}, 1,16\}]]$

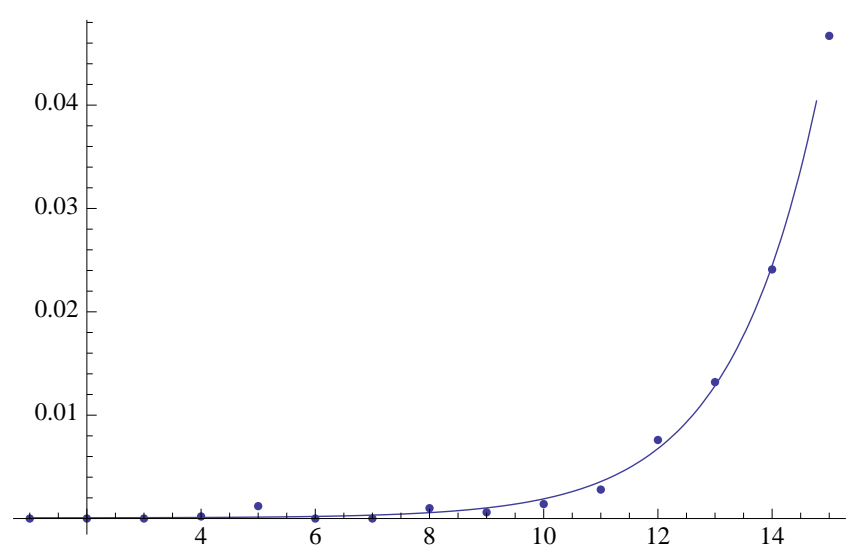

$\mathrm{x} 213 \mathrm{exp}=$

NonlinearModelFit [X213data $[2 ; ; 15]], b+d 0 * a \wedge x,\{b, d 0, a\}, \mathbf{x}$, MaxIterations $\rightarrow 1000]$

FittedModel $\left[2.46294 \times 10^{-6}+8.81896 \times 10^{-7} \ll 18 \gg^{x}\right]$

X213exp ["BestFit"]

$2.46294 \times 10^{-6}+8.81896 \times 10^{-7} 2.02643^{x}$ 
Show [ListPlot $[\mathrm{X} 213 \operatorname{dat}[[1 ; ; 15]], \mathrm{PlotRange} \rightarrow \mathrm{All}], \mathrm{P} 1$ ot $[\mathrm{X} 213 \exp [\mathbf{x}],\{\mathbf{x}, 1,17\}]]$

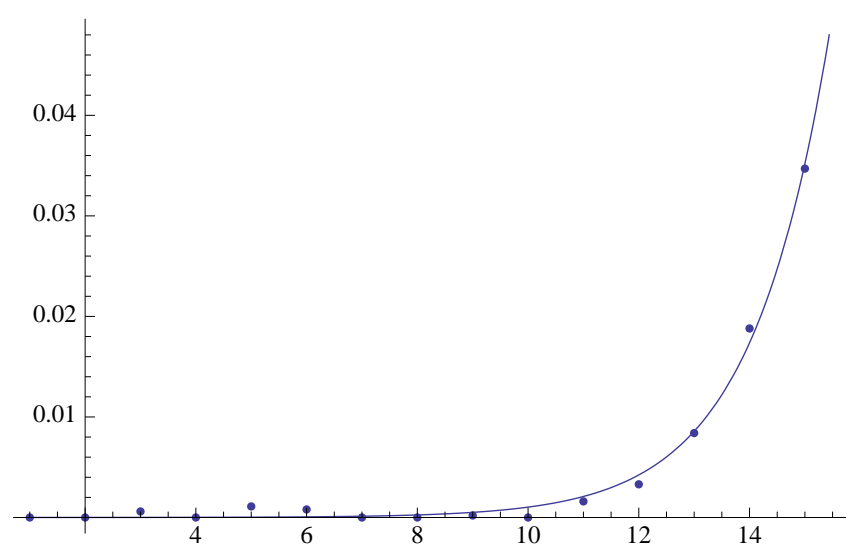

$\mathrm{X} 214 \mathrm{exp}=$

NonlinearModelFit $[\mathbf{X} 214$ data $[2 ; ; 15]], b+d 0 * a^{\wedge} \mathbf{x},\{b, d 0, a\}, \mathbf{x}$, MaxIterations $\left.\rightarrow 1000\right]$

FittedModel $\left[0.000103384+3.18872 \times 10^{-6} \ll 19 \gg^{x}\right]$

X214exp ["BestFit"]

$0.000103384+3.18872 \times 10^{-6} 1.86465^{x}$

Show [ListPlot $[X 214$ data $[1 ; ; 20]], P 1$ otRange $\rightarrow A l 1], P 1 o t[X 214 \exp [\mathbf{x}],\{x, 1,22\}]]$

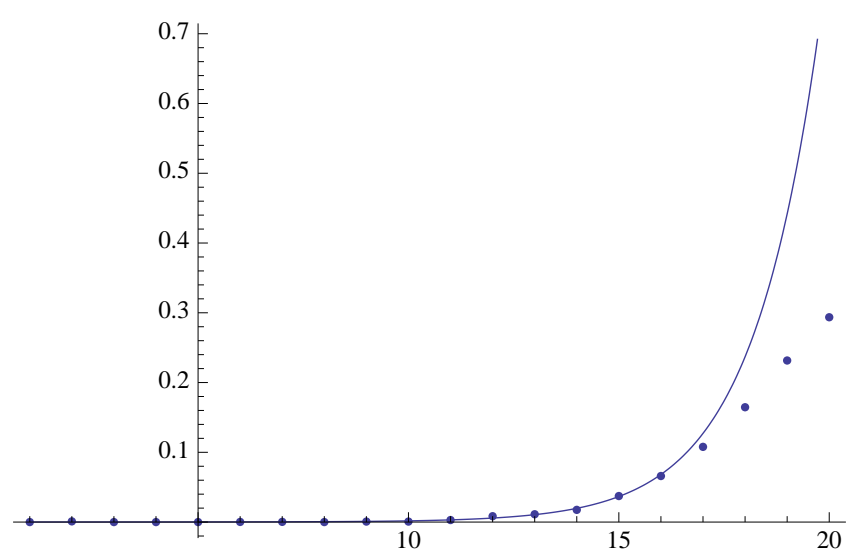

$\mathrm{X} 221 \mathrm{exp}=$

NonlinearModelFit $\left[\mathbf{X 2 2 1 d a t a}[[1 ; ; 15]], b+d 0 * a^{\wedge} \mathbf{x},\{b, d 0, a\}, \mathbf{x}\right.$, MaxIterations $\left.\rightarrow 1000\right]$

FittedModel $\left[0.0000345803+0.0000176973 \ll 19>^{x}\right]$

X221exp ["BestFit"]

$0.0000345803+0.00001769731 .66956^{x}$ 
Show [ListPlot $[\mathrm{X} 221$ data $[[1 ; ; 20]], \mathrm{PlotRange} \rightarrow \mathrm{All}], \mathrm{P} 1$ ot $[\mathrm{X} 221 \exp [\mathbf{x}],\{\mathbf{x}, 1,20\}]]$

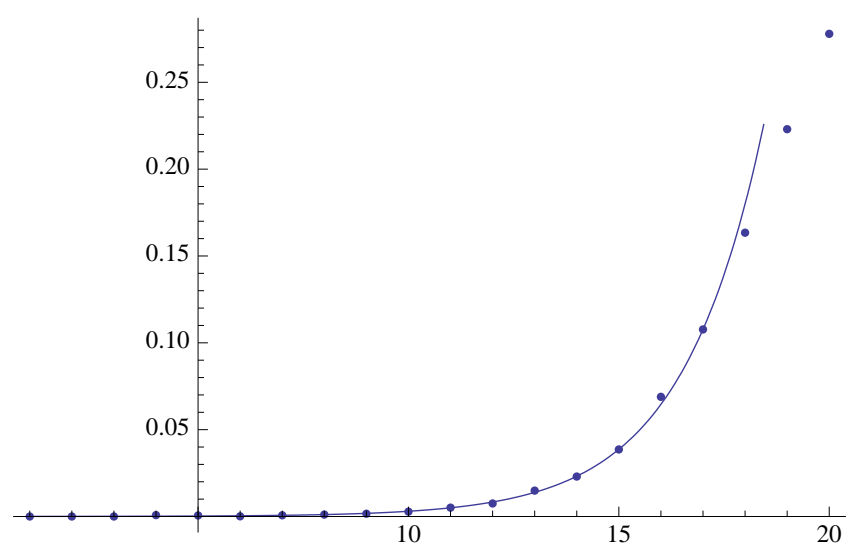

$\mathrm{x} 222 \mathrm{exp}=$

NonlinearModelFit [X222data $[1 ; ; 15]], b+d 0 * a^{\wedge} \mathbf{x},\{b, d 0, a\}, \mathbf{x}$, MaxIterations $\left.\rightarrow 1000\right]$

FittedModel $\left[0.000543117+8.4109 \times 10^{-6} \ll 19 \gg^{x}\right]$

X222exp ["BestFit"]

$0.000543117+8.4109 \times 10^{-6} 1.74762^{x}$

Show [ListPlot $[\mathrm{X} 222 \operatorname{dat} a[1 ; ; 15]], \mathrm{PlotRange} \rightarrow \mathrm{All}], \mathrm{Plot}[\mathrm{X} 222 \exp [\mathbf{x}],\{\mathbf{x}, 1,16\}]]$

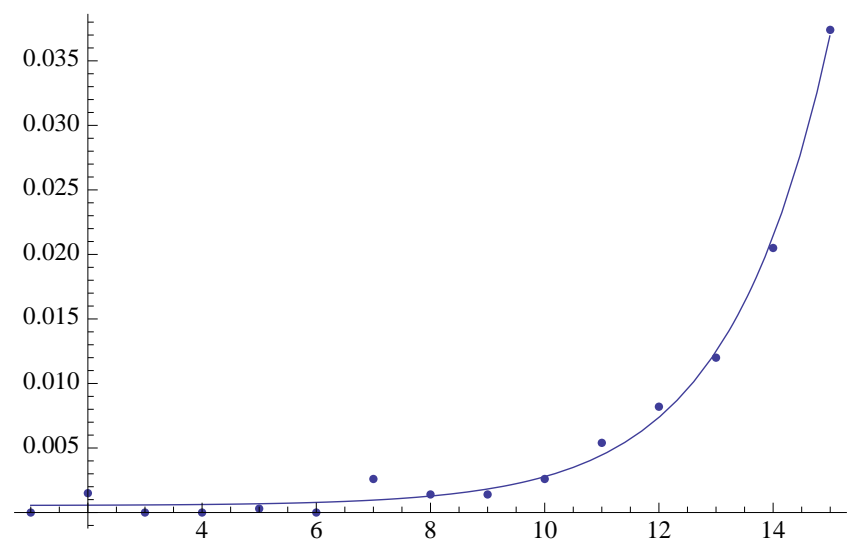

$\mathrm{x} 223 \mathrm{exp}=$

NonlinearModelFit [X223data $[[2 ; ; 15]], b+d 0 * a \wedge x,\{b, d 0, a\}, \mathbf{x}$, MaxIterations $\rightarrow 1000]$

FittedModel $\left[-0.000158784+6.77524 \times 10^{-6} \ll 19 \gg^{x}\right]$

X223exp ["BestFit"]

$-0.000158784+6.77524 \times 10^{-6} 1.77526^{x}$ 
Show [ListPlot $[\mathrm{X} 223 \operatorname{dat} a[1 ; ; 15]], \mathrm{PlotRange} \rightarrow \mathrm{All}], \mathrm{Plot}[\mathrm{X} 223 \exp [\mathbf{x}],\{\mathbf{x}, 1,17\}]]$

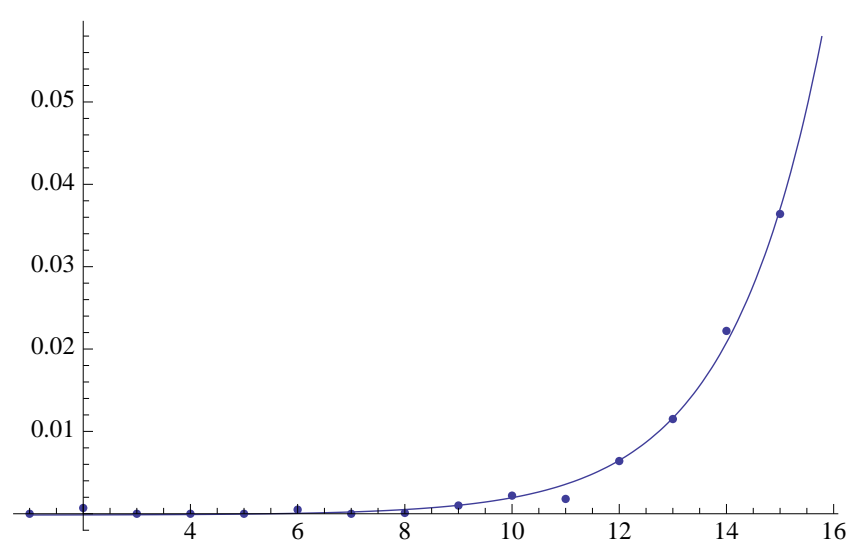

$\mathrm{X} 224 \mathrm{exp}=$

NonlinearModelFit $\left[\mathbf{X} 224\right.$ data $[[2 ; ; 15]], b+d 0 * a^{\wedge} \mathbf{x},\{b, d 0, a\}, \mathbf{x}, \operatorname{Max}$ Iterations $\left.\rightarrow 1000\right]$

FittedModel $\left[0.000588167+5.36974 \times 10^{-6} \ll 19 \gg^{x}\right]$

$\mathrm{x} 224 \exp [$ "BestFit"]

$0.000588167+5.36974 \times 10^{-6} 1.80439^{x}$

Show $[$ ListPlot $[X 224$ data $[[1 ; ; 20]], P l o t R a n g e \rightarrow A l l], P 1 o t[X 224 \exp [x],\{x, 1,22\}]]$

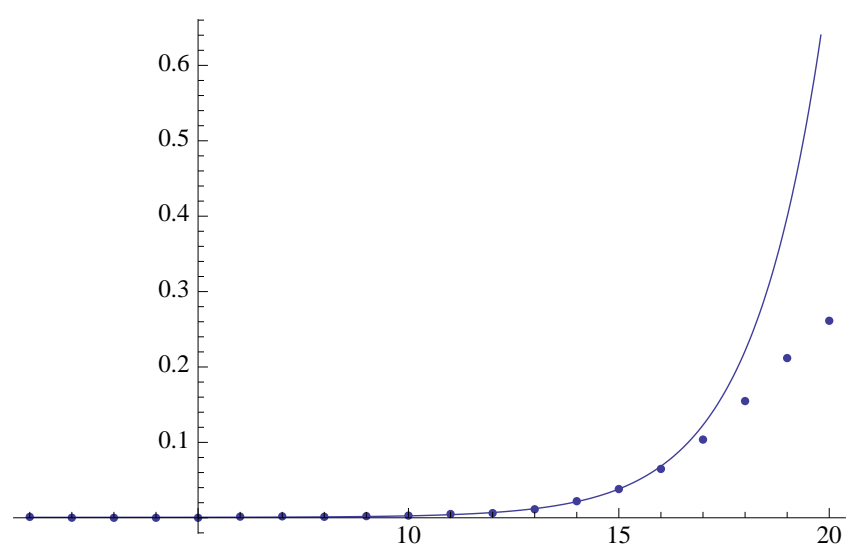

$\mathrm{x} 231 \mathrm{exp}=$

NonlinearModelFit $\left[\mathrm{X} 231\right.$ data $[[1 ; ; 15]], b+d 0 * a^{\wedge} \mathbf{x},\{b, d 0, a\}, \mathbf{x}$, MaxIterations $\left.\rightarrow 1000\right]$

FittedModel $\left[-0.0000314852+9.43912 \times 10^{-6} \ll 19 \gg^{x}\right]$

$\mathrm{x} 231 \exp [$ "BestFit"]

$-0.0000314852+9.43912 \times 10^{-6} 1.74424^{x}$ 
Show [ListPlot $[\mathrm{X} 231$ data $[1 ; ; 20]], \mathrm{PlotRange} \rightarrow \mathrm{All}], \mathrm{P} 1$ ot $[\mathrm{X} 231 \exp [\mathbf{x}],\{\mathbf{x}, 1,20\}]]$

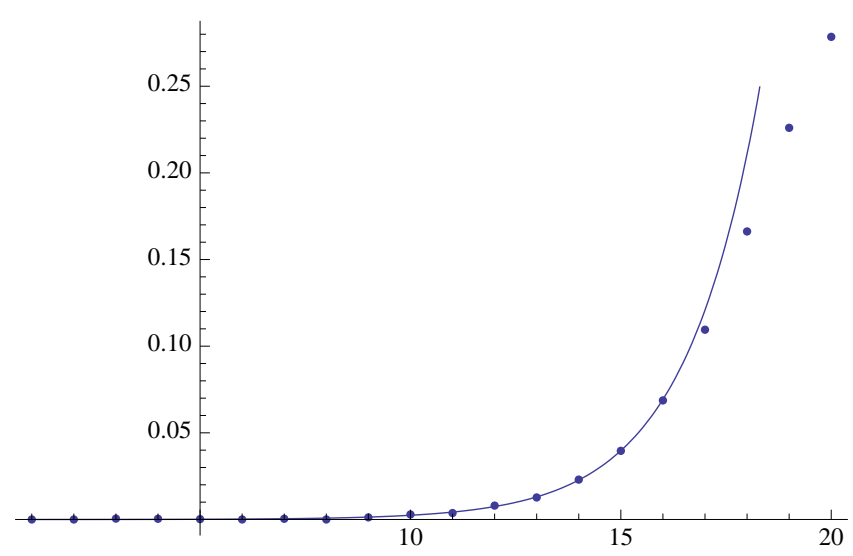

$\mathrm{x} 232 \mathrm{exp}=$

NonlinearModelFit [X232data $[1 ; ; 15]], b+d 0 * a \wedge x,\{b, d 0, a\}, \mathbf{x}$, MaxIterations $\rightarrow 1000]$

FittedModel $\left[0.000101681+6.19129 \times 10^{-6} \ll 19 \gg^{x}\right]$

$\mathrm{x} 232 \exp [$ "BestFit"]

$0.000101681+6.19129 \times 10^{-6} 1.78595^{x}$

Show $[$ ListPlot $[\mathrm{X} 232 \operatorname{data}[[1 ; ; 15]], \mathrm{PlotRange} \rightarrow \mathrm{All}], \mathrm{P} 1$ ot $[\mathrm{X} 232 \exp [\mathbf{x}],\{\mathbf{x}, 1,16\}]]$

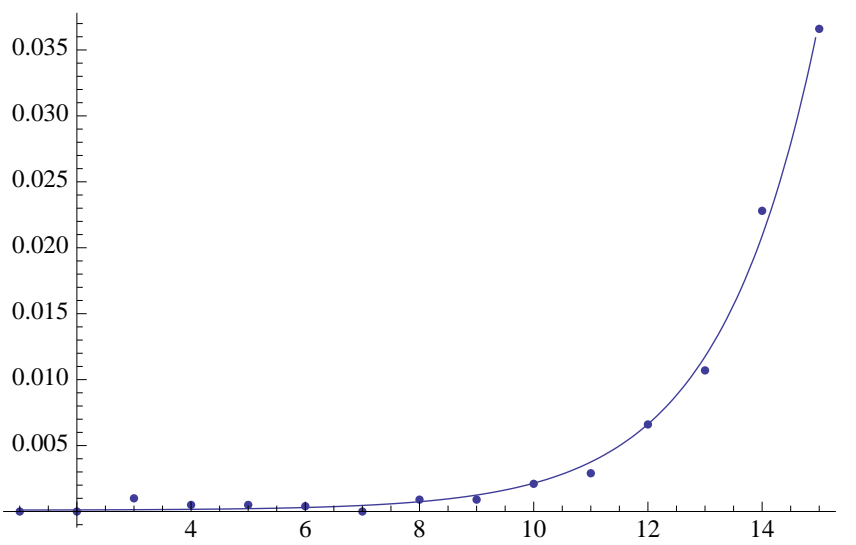

$\mathrm{x} 233 \mathrm{exp}=$

NonlinearModelFit [X233data $[2 ; ; 15]], b+d 0 * a \wedge x,\{b, d 0, a\}, \mathbf{x}$, MaxIterations $\rightarrow 1000]$

FittedModel $\left[0.000396906+0.0000190444 \ll 19 \gg^{x}\right]$

X233exp ["BestFit"]

$0.000396906+0.00001904441 .65027^{x}$ 
Show [ListPlot $[\mathrm{X} 233 \operatorname{data}[[1 ; ; 15]], \mathrm{PlotRange} \rightarrow \mathrm{All}], \mathrm{P} 1$ ot $[\mathrm{X} 233 \exp [\mathbf{x}],\{\mathbf{x}, 1,17\}]]$

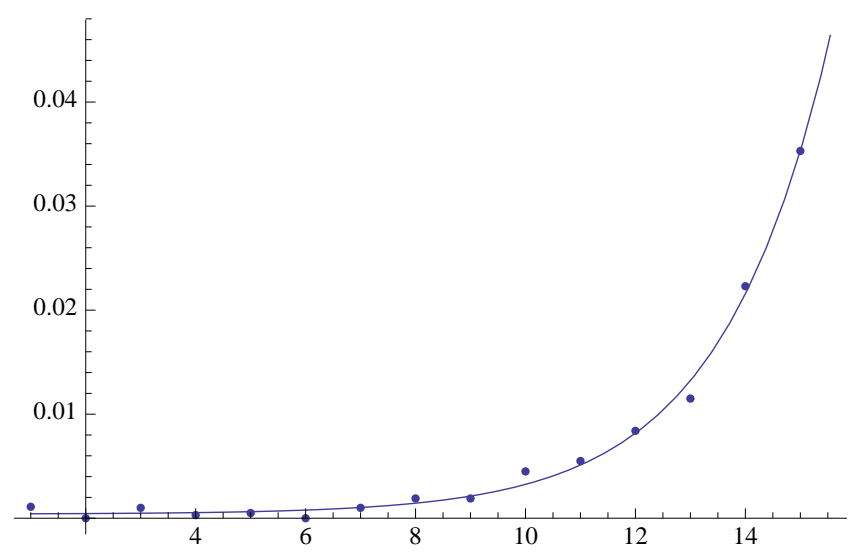

$\mathrm{X} 234 \mathrm{exp}=$

NonlinearModelFit $[\mathbf{x} 234$ data $[[2 ; ; 15]], b+d 0 * a \wedge x,\{b, d 0, a\}, \mathbf{x}, \operatorname{MaxIterations} \rightarrow 1000]$

FittedModel $\left[0.000286217+5.04736 \times 10^{-6} \ll 19 \gg^{x}\right]$

$\mathrm{x} 234 \exp$ ["BestFit"]

$0.000286217+5.04736 \times 10^{-6} 1.80303^{x}$

Show $[$ ListPlot $[\mathrm{X} 234$ data $[[1 ; ; 20]], \mathrm{PlotRange} \rightarrow \mathrm{All}], \mathrm{Plot}[\mathrm{X} 234 \exp [\mathrm{x}],\{\mathrm{x}, 1,22\}]]$

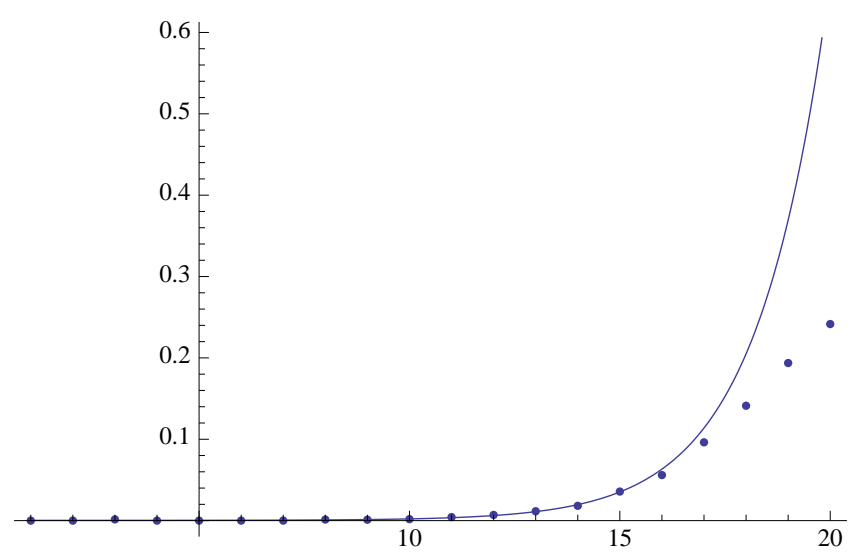

$\mathrm{X} 241 \mathrm{exp}=$

NonlinearModelFit $[\mathbf{X} 241$ data $[1 ; ; 15]], b+d 0 * a^{\wedge} \mathbf{x},\{b, d 0, a\}, \mathbf{x}$, MaxIterations $\left.\rightarrow 1000\right]$

FittedModel $\left[0.0000187071+0.0000100793 \ll 19>^{x}\right]$

x241exp [ BestFit"]

$0.0000187071+0.00001007931 .75597^{x}$ 
Show [ListPlot $[\mathrm{X} 241$ data $[1 ; ; 20]], \mathrm{PlotRange} \rightarrow \mathrm{All}], \mathrm{P} 1$ ot $[\mathrm{X} 241 \exp [\mathbf{x}],\{\mathbf{x}, 1,20\}]]$

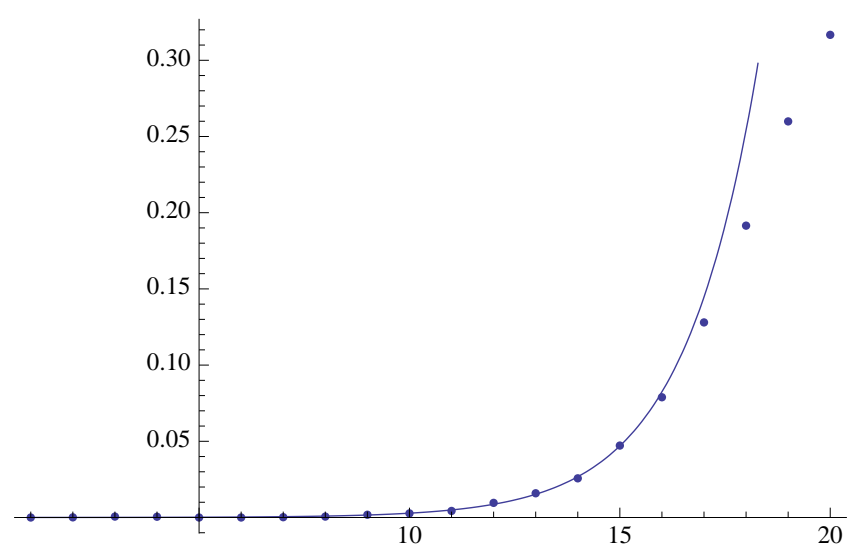

$\mathrm{x} 242 \mathrm{exp}=$

NonlinearModelFit [X242data $[1 ; ; 15]], b+d 0 * a^{\wedge} \mathbf{x},\{b, d 0, a\}, \mathbf{x}$, MaxIterations $\left.\rightarrow 1000\right]$

FittedModel $\left[0.000103583+5.04605 \times 10^{-6} \ll 19 \gg^{x}\right]$

$\mathrm{x} 242 \exp [$ "BestFit"]

$0.000103583+5.04605 \times 10^{-6} 1.81483^{x}$

Show $[$ ListPlot $[\mathrm{X} 242 \operatorname{dat}[[1 ; ; 15]], \mathrm{P}$ lotRange $\rightarrow \mathrm{All}], \mathrm{P} 1$ ot $[\mathrm{X} 242 \exp [\mathrm{x}],\{\mathrm{x}, 1,16\}]]$

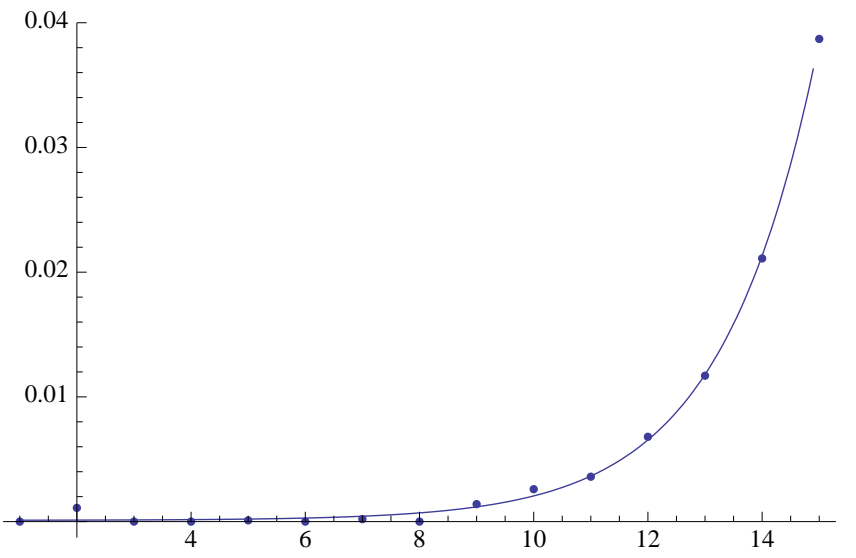

$\mathrm{X} 243 \mathrm{exp}=$

NonlinearModelFit [X243data $[[2 ; 15]], b+d 0 * a^{\wedge} \mathbf{x},\{b, d 0, a\}, \mathbf{x}$, MaxIterations $\left.\rightarrow 1000\right]$

FittedModel $\left[-0.0000868314+5.18919 \times 10^{-6} \ll 19 \gg^{x}\right]$

X243exp ["BestFit"]

$-0.0000868314+5.18919 \times 10^{-6} 1.78797^{x}$ 
Show [ListPlot $[\mathrm{X} 243 \operatorname{dat}[[1 ; ; 15]], \mathrm{PlotRange} \rightarrow \mathrm{All}], \mathrm{Plot}[\mathrm{X} 243 \exp [\mathrm{x}],\{\mathbf{x}, 1,17\}]]$

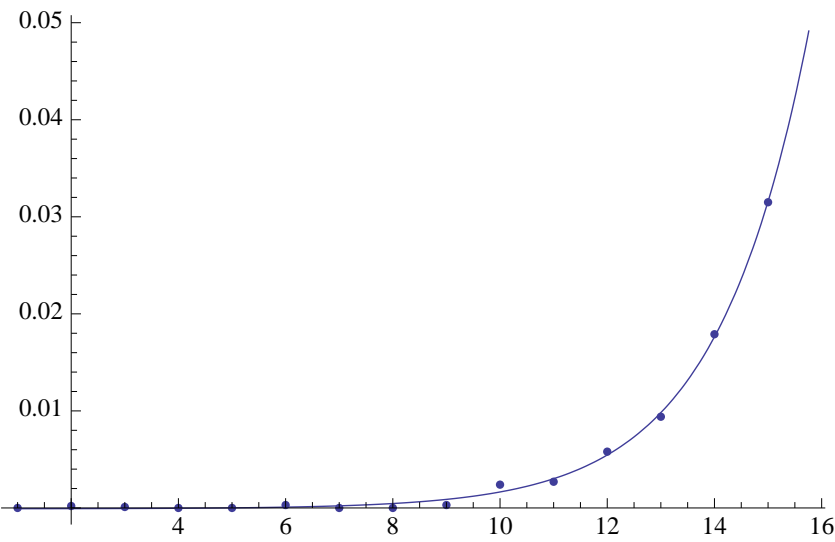

$\mathrm{X} 244 \mathrm{exp}=$

NonlinearModelFit [X244data $[2 ; ; 15]], b+d 0 * a^{\wedge} \mathbf{x},\{b, d 0, a\}, \mathbf{x}$, MaxIterations $\left.\rightarrow 1000\right]$

FittedModel $\left[-6.79183 \times 10^{-6}+4.03432 \times 10^{-6} \ll 19 \gg^{x}\right]$

X244exp [ BestFit"]

$-6.79183 \times 10^{-6}+4.03432 \times 10^{-6} 1.81664^{x}$

Show [ListPlot $[X 244$ data $[1 ; ; 20]], P 1$ otRange $\rightarrow A l 1], P 1 o t[X 244 \exp [x],\{x, 1,22\}]]$

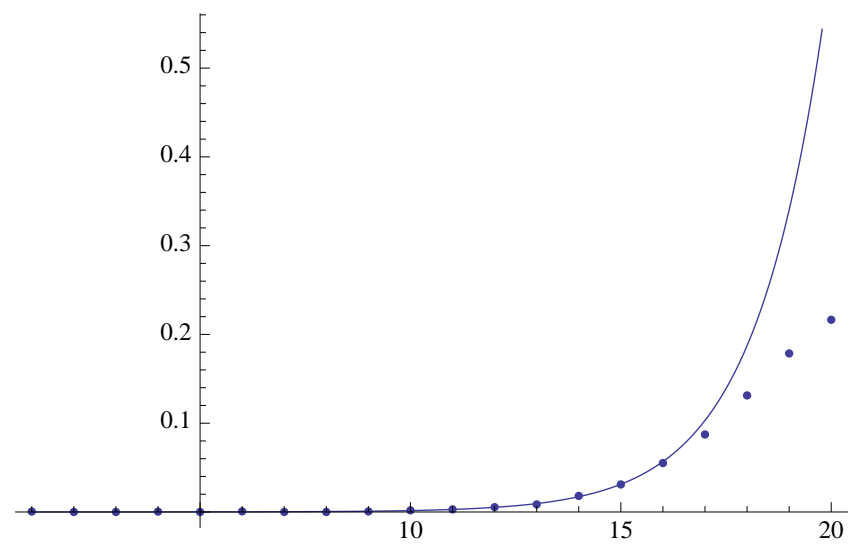

$\mathrm{X} 251 \mathrm{exp}=$

NonlinearModelFit [X251data $[1 ; ; ; 15]], b+d 0 * a \wedge x,\{b, d 0, a\}, \mathbf{x}$, MaxIterations $\rightarrow 1000]$

FittedModel $\left[0.000279727+0.0000186937 \ll 19>^{x}\right]$

X251exp ["BestFit"]

$0.000279727+0.00001869371 .66883^{x}$ 
Show [ListPlot $[\mathrm{X} 251$ data $[[1 ; ; 20]], \mathrm{PlotRange} \rightarrow \mathrm{All}], \mathrm{P} 1$ ot $[\mathrm{X} 251 \exp [\mathbf{x}],\{\mathbf{x}, 1,20\}]]$

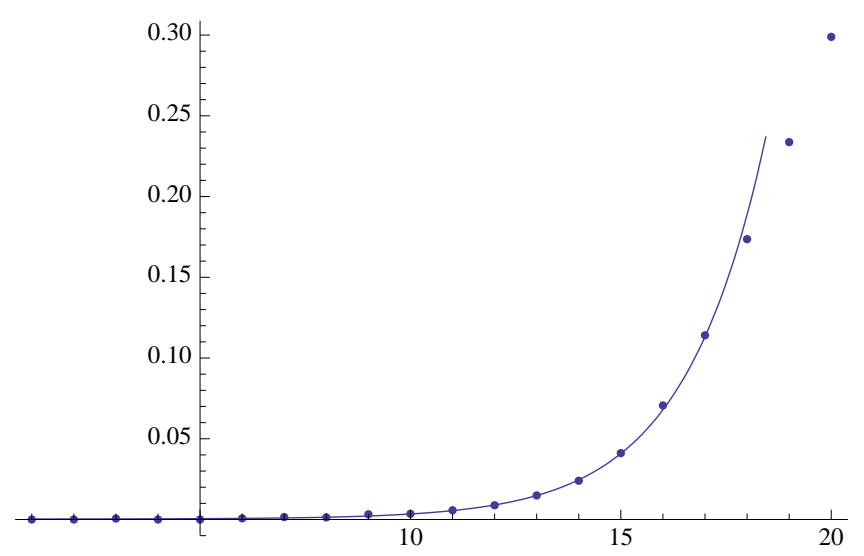

$\mathrm{x} 252 \mathrm{exp}=$

NonlinearModelFit [X252data $[1 ; ; ; 15]], b+d 0 * a^{\wedge} \mathbf{x},\{b, d 0, a\}, \mathbf{x}$, MaxIterations $\left.\rightarrow 1000\right]$

FittedModel $\left[0.0000186645+9.61072 \times 10^{-6} \ll 19 \gg^{x}\right]$

$\mathrm{x} 252 \exp [$ "BestFit"]

$0.0000186645+9.61072 \times 10^{-6} 1.74947^{x}$

Show $[$ ListPlot $[X 252 \operatorname{data}[[1 ; 15]], \mathrm{PlotRange} \rightarrow \mathrm{All}], \mathrm{P} 1$ ot $[\mathrm{X} 252 \exp [\mathbf{x}],\{\mathbf{x}, 1,16\}]]$

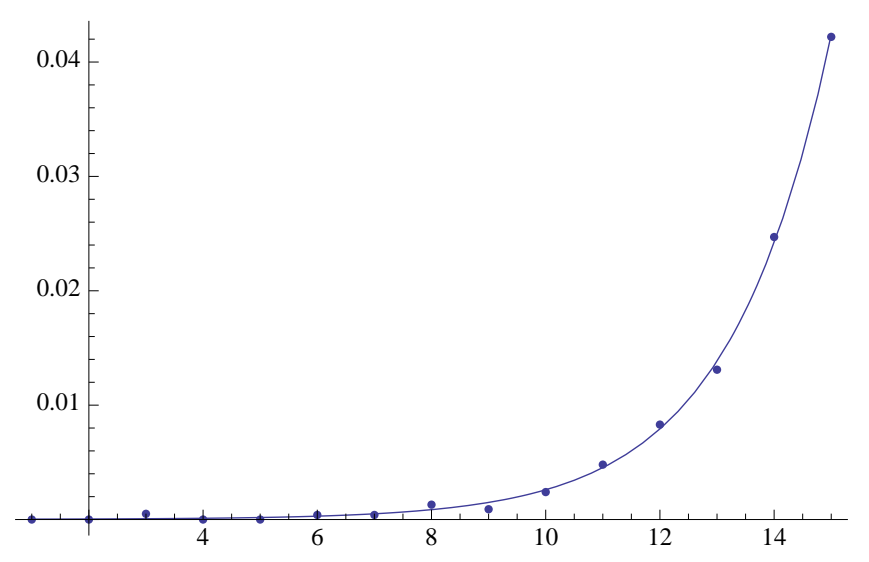

$\mathrm{x} 253 \mathrm{exp}=$

NonlinearModelFit [X253data $[[2 ; ; 15]], b+d 0 * a \wedge \mathbf{x},\{b, d 0, a\}, \mathbf{x}$, MaxIterations $\rightarrow 1000]$

FittedModel $\left[0.0000711672+5.78513 \times 10^{-6} \ll 19 \gg^{x}\right]$

X253exp ["BestFit"]

$0.0000711672+5.78513 \times 10^{-6} 1.78574^{x}$ 


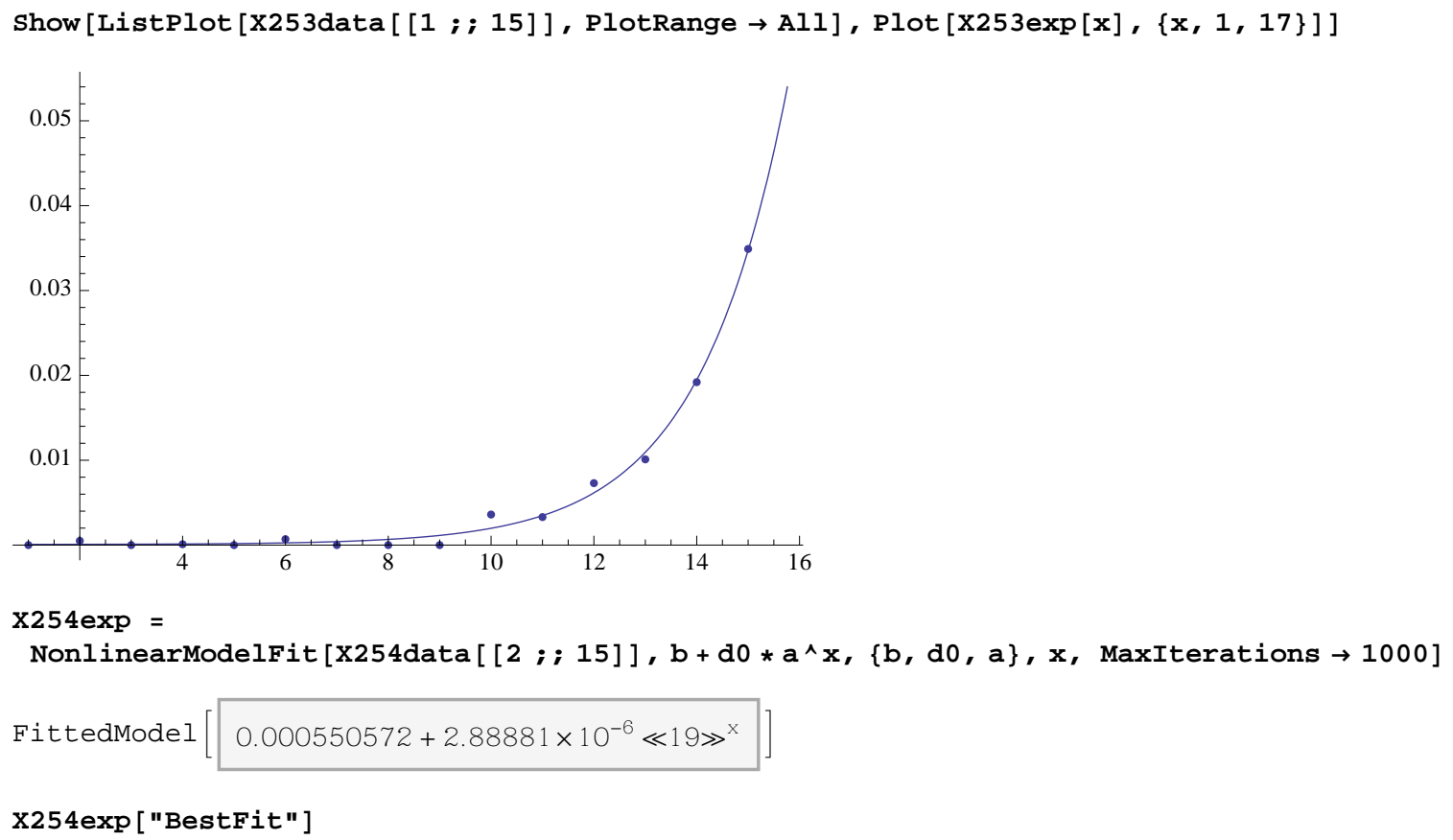

$0.000550572+2.88881 \times 10^{-6} 1.87563^{x}$

Show $[$ ListPlot $[\mathrm{X} 254$ data $[[1 ; ; 20]], \mathrm{PlotRange} \rightarrow \mathrm{All}], \mathrm{P} 1$ lot $[\mathrm{X} 254 \exp [\mathbf{x}],\{\mathbf{x}, 1,22\}]]$

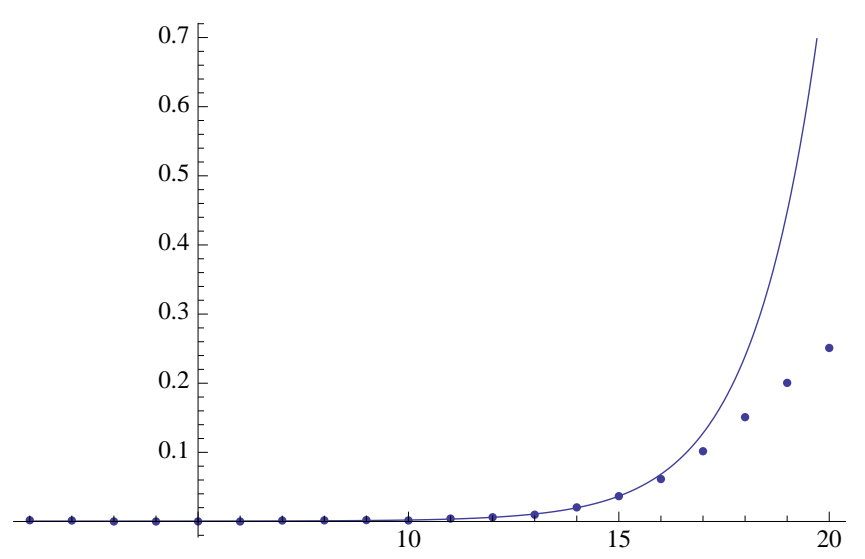

$\mathrm{x} 311 \mathrm{exp}=$

NonlinearModelFit $[x 311$ data $[[2 ; ; 19]], b+d 0 * a \wedge x,\{b, d 0, a\}, \mathbf{x}$, MaxIterations $\rightarrow 1000]$

FittedModel $\left[0.000297039+2.37729 \times 10^{-7} \ll 18 \gg^{x}\right]$

X311exp ["BestFit"]

$0.000297039+2.37729 \times 10^{-7} 1.88632^{x}$ 
Show [ListPlot $[\mathrm{X} 311$ data $[1 ; ; 19]], \mathrm{PlotRange} \rightarrow \mathrm{All}], \mathrm{Plot}[\mathrm{X} 311 \exp [\mathbf{x}],\{\mathbf{x}, 1,21\}]]$

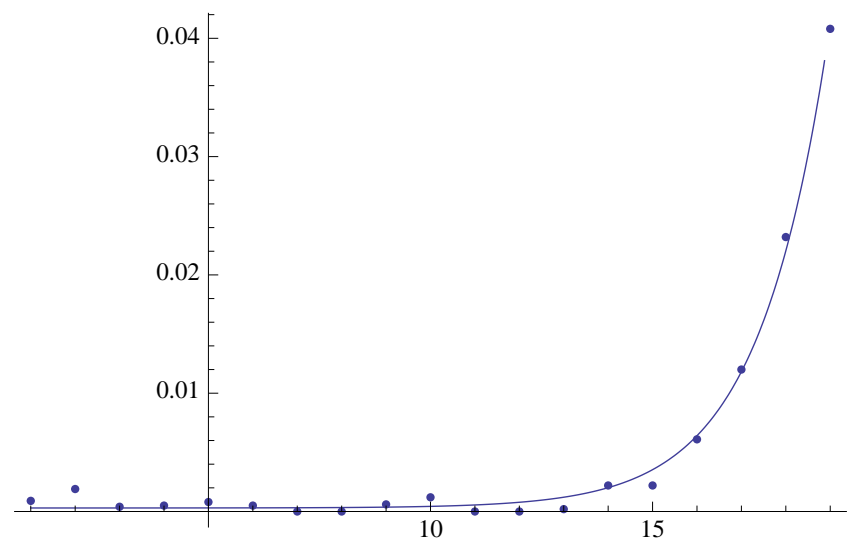

$\mathrm{x} 312 \mathrm{exp}=$

NonlinearModelFit [x312data $[[2 ; ; 19]], b+d 0 * a \wedge x,\{b, d 0, a\}, \mathbf{x}$, MaxIterations $\rightarrow 1000]$

FittedModel $\left[0.000161324+7.06402 \times 10^{-7} \ll 19 \gg^{x}\right]$

X312exp ["BestFit"]

$0.000161324+7.06402 \times 10^{-7} 1.78783^{x}$

Show [ListPlot $[\mathrm{X} 312 \operatorname{dat} a[1 ; ; 19]], \mathrm{PlotRange} \rightarrow \mathrm{All}], \mathrm{Plot}[\mathrm{X} 312 \exp [\mathbf{x}],\{\mathbf{x}, 1,21\}]]$

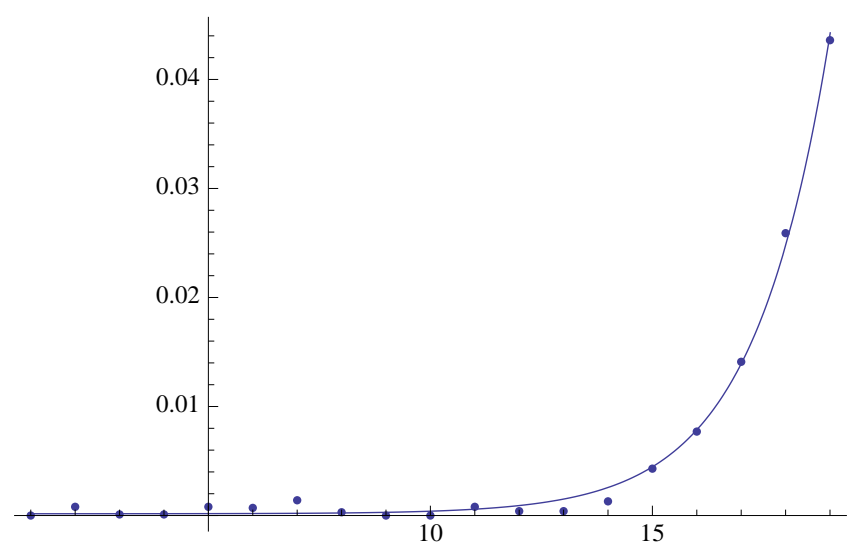

$\mathrm{x} 313 \mathrm{exp}=$

NonlinearModelFit [X313data $[[2 ; ; 19]], b+d 0 * a \wedge x,\{b, d 0, a\}, \mathbf{x}$, MaxIterations $\rightarrow 1000]$

FittedModel $\left[-0.000109957+1.40857 \times 10^{-6} \ll 19 \gg^{\times}\right]$

x313exp ["BestFit"]

$-0.000109957+1.40857 \times 10^{-6} 1.70873^{x}$ 
Show [ListPlot $[\mathrm{X313data}[[1 ; ; 19]], \mathrm{PlotRange} \rightarrow \mathrm{All}], \mathrm{Plot}[\mathrm{X313exp}[\mathbf{x}],\{\mathbf{x}, 1,21\}]]$

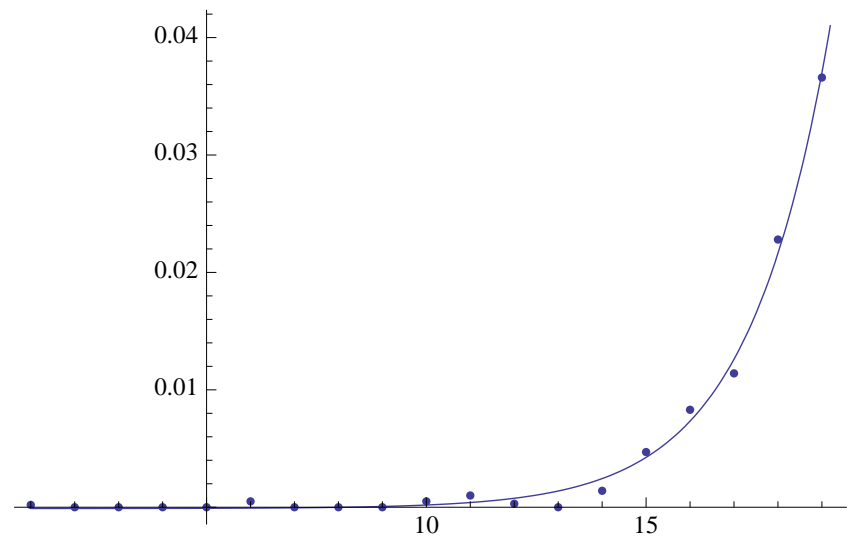

$\mathrm{X} 314 \mathrm{exp}=$

NonlinearModelFit $[\mathbf{x} 314$ data $[2 ; ; 19]], b+d 0 * a^{\wedge} \mathbf{x},\{b, d 0, a\}, \mathbf{x}$, MaxIterations $\left.\rightarrow 1000\right]$

FittedModel $\left[0.000290109+7.68663 \times 10^{-7} \ll 19 \gg^{x}\right]$

x314exp ["BestFit"]

$0.000290109+7.68663 \times 10^{-7} 1.76487^{x}$

Show [ListPlot $[\mathrm{X} 314 \operatorname{data}[[1 ; ; 19]], \mathrm{PlotRange} \rightarrow \mathrm{All}], \mathrm{Plot}[\mathrm{X} 314 \exp [\mathrm{x}],\{\mathrm{x}, 1,21\}]]$

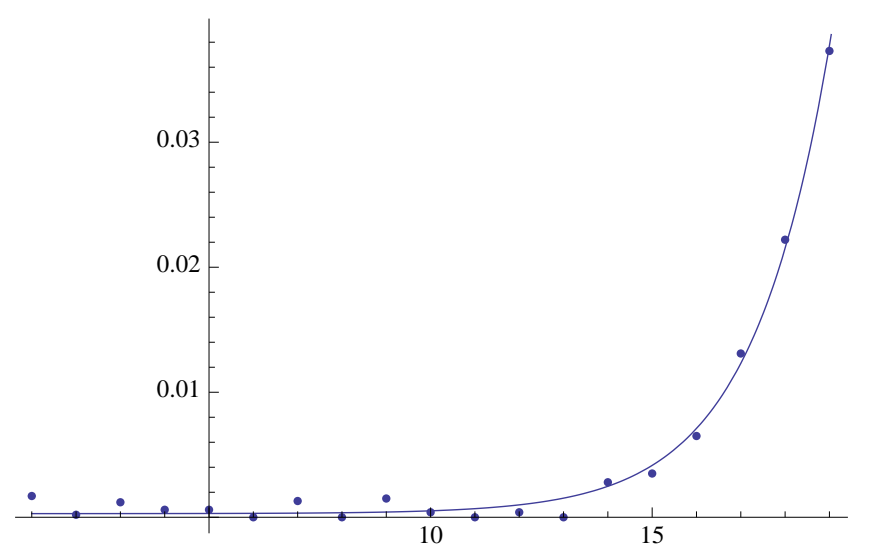

$\mathrm{x} 321 \mathrm{exp}=$

NonlinearModelFit [x321data $[2 ; ; 19]], b+d 0 * a \wedge x,\{b, d 0, a\}, \mathbf{x}$, MaxIterations $\rightarrow 1000]$

FittedModel $\left[-0.000171617+5.09911 \times 10^{-7} \ll 19 \gg^{x}\right]$

x321exp ["BestFit"]

$-0.000171617+5.09911 \times 10^{-7} 1.80669^{x}$ 
Show [ListPlot $[\mathrm{X} 321$ data $[1 ; ; 20]], \mathrm{PlotRange} \rightarrow \mathrm{All}], \mathrm{Plot}[\mathrm{X} 321 \exp [\mathbf{x}],\{\mathbf{x}, 1,20\}]]$

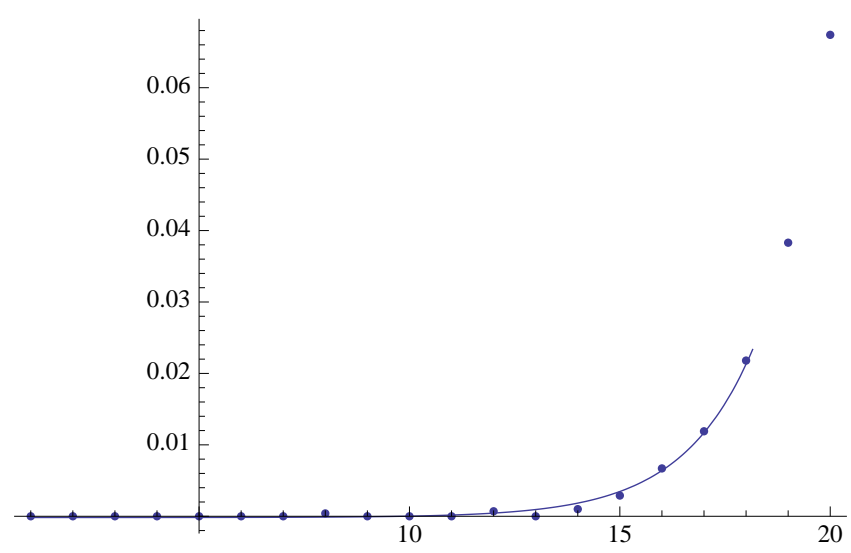

$\mathrm{x} 322 \mathrm{exp}=$

NonlinearModelFit [X322data $[[2 ; ; 19]], b+d 0 * a \wedge x,\{b, d 0, a\}, \mathbf{x}$, MaxIterations $\rightarrow 1000]$

FittedModel $\left[-0.000117151+5.72873 \times 10^{-7} \ll 18 \gg^{x}\right]$

X322exp ["BestFit"]

$-0.000117151+5.72873 \times 10^{-7} 1.80331^{x}$

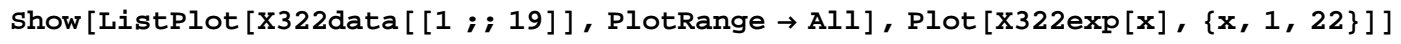

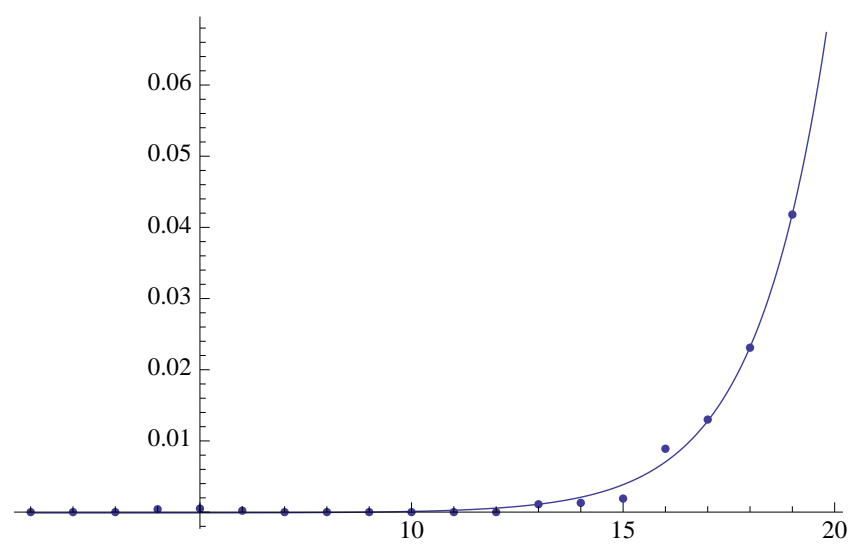

$\mathrm{x} 323 \mathrm{exp}=$

NonlinearModelFit $\left[\mathrm{x} 323\right.$ data $[[2 ; ; 19]], b+d 0 * a^{\wedge} \mathbf{x},\{b, d 0, a\}, \mathbf{x}$, MaxIterations $\left.\rightarrow 1000\right]$

FittedModel $\left[-0.0000529368+8.26306 \times 10^{-7} \ll 18 \gg^{x}\right]$

X323exp ["BestFit"]

$-0.0000529368+8.26306 \times 10^{-7} 1.78125^{x}$ 


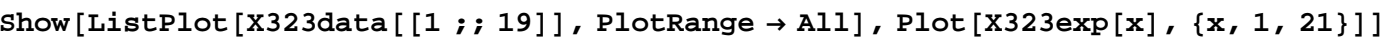

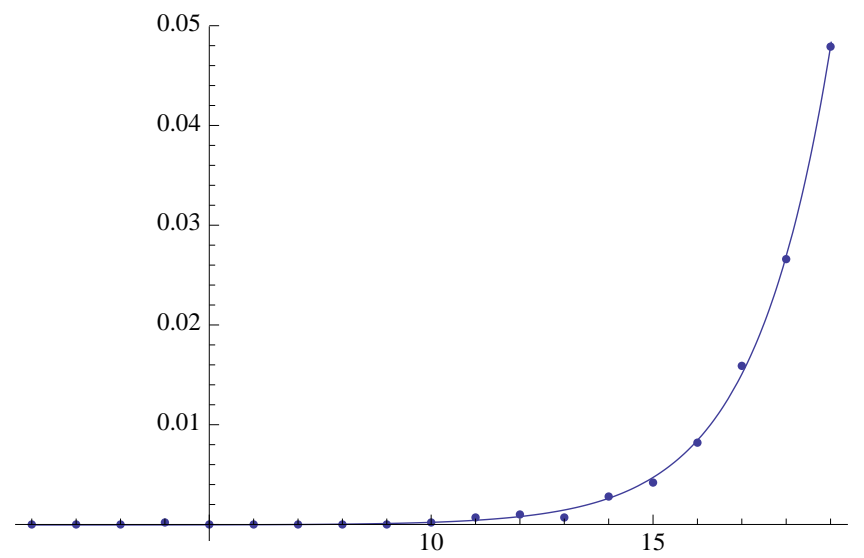

$\mathrm{x} 324 \mathrm{exp}=$

NonlinearModelFit $[\mathbf{x} 324$ data $[2 ; ; 19]], b+d 0 * a^{\wedge} \mathbf{x},\{b, d 0, a\}, \mathbf{x}$, MaxIterations $\left.\rightarrow 1000\right]$

FittedModel $\left[-0.000136721+6.20397 \times 10^{-7} \ll 19 \gg^{x}\right]$

x324exp ["BestFit"]

$-0.000136721+6.20397 \times 10^{-7} 1.81211^{x}$

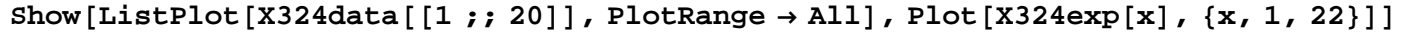

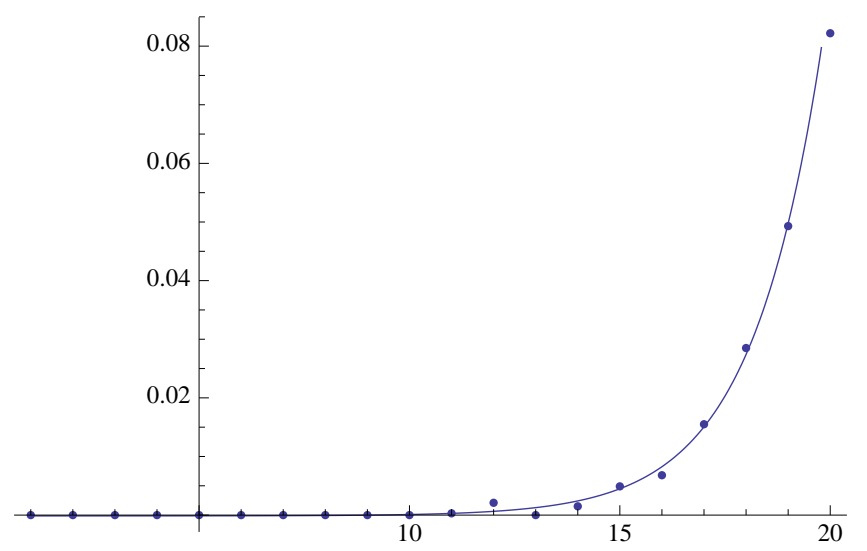

$\mathrm{X} 331 \mathrm{exp}=$

NonlinearModelFit $[\mathbf{x 3 3 1 d a t a [}[2 ; ; 19]], b+d 0 * a^{\wedge} \mathbf{x},\{b, d 0, a\}, \mathbf{x}$, MaxIterations $\left.\rightarrow 1000\right]$

FittedModel $\left[-0.0000362272+4.23537 \times 10^{-7} \ll 18 \gg^{x}\right]$

x331exp ["BestFit"]

$-0.0000362272+4.23537 \times 10^{-7} 1.84691^{x}$ 


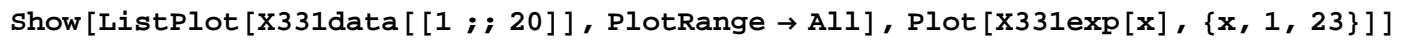

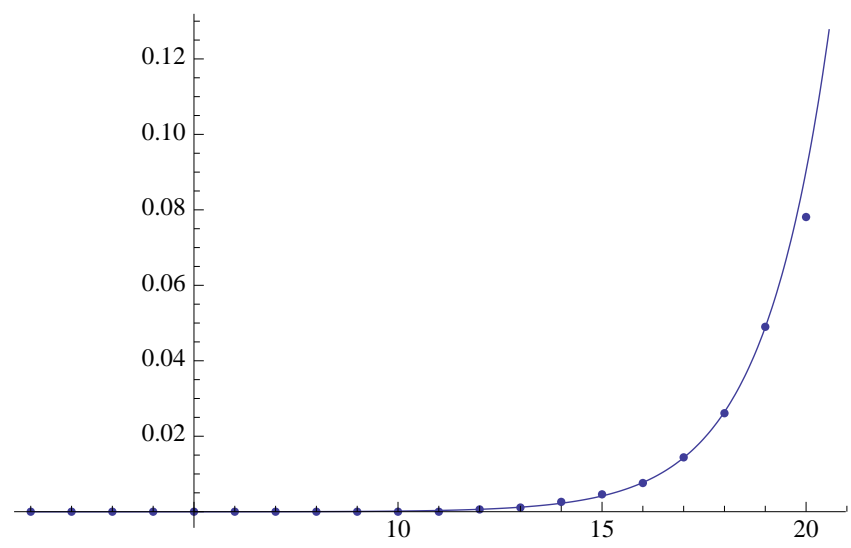

$\mathrm{x} 332 \mathrm{exp}=$

NonlinearModelFit [X332data $[[10 ; ; 19]], b+d 0 * a^{\wedge} \mathbf{x},\{b, d 0, a\}, \mathbf{x}$, MaxIterations $\left.\rightarrow 1000\right]$

FittedModel $\left[-0.000677626+6.47734 \times 10^{-7} \ll 19 \gg^{x}\right]$

x332exp ["BestFit"]

$-0.000677626+6.47734 \times 10^{-7} 1.82007^{x}$

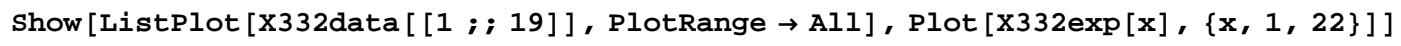

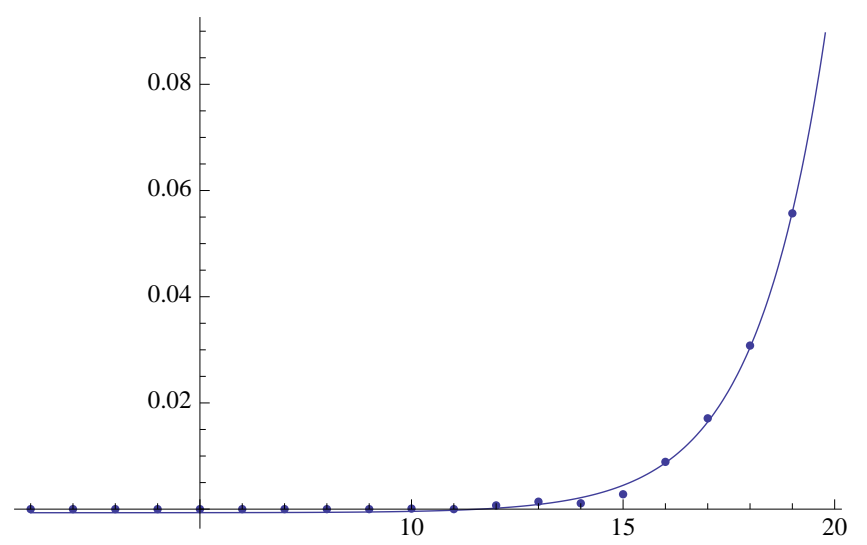

$\mathrm{x} 333 \mathrm{exp}=$

NonlinearModelFit $\left[\mathrm{X333data}[[2 ; ; 19]], b+d 0 * a^{\wedge} \mathbf{x},\{b, d 0, a\}, \mathbf{x}\right.$, MaxIterations $\left.\rightarrow 1000\right]$

FittedModel $\left[-0.00014129+6.76233 \times 10^{-7} \ll 19 \gg^{x}\right]$

x333exp ["BestFit"]

$-0.00014129+6.76233 \times 10^{-7} 1.80744^{x}$ 
Show $[$ ListPlot $[\mathrm{X} 333$ data $[1 ; ; 19]], \mathrm{PlotRange} \rightarrow \mathrm{All}], \mathrm{P} \operatorname{lot}[\mathrm{X} 333 \exp [\mathbf{x}],\{\mathbf{x}, 1,22\}]$

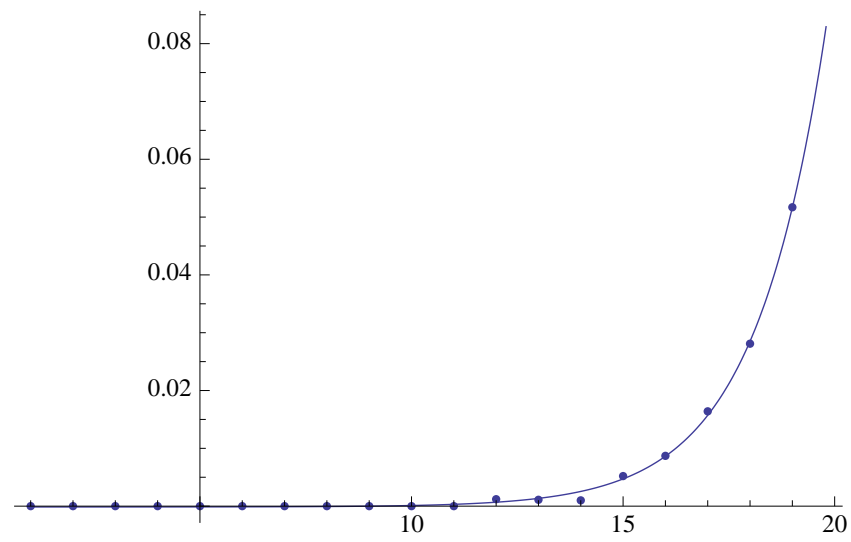

$\mathrm{x} 334 \mathrm{exp}=$

NonlinearModelFit $[\mathrm{X} 334$ data $[10 ; ; 19]], b+d 0 * a \wedge \mathbf{x},\{b, d 0, a\}, \mathbf{x}$, MaxIterations $\rightarrow 1000]$

FittedModel $\left[-0.000581152+1.2912 \times 10^{-6} \ll 19 \gg^{x}\right]$

x334exp ["BestFit"]

$-0.000581152+1.2912 \times 10^{-6} 1.754^{x}$

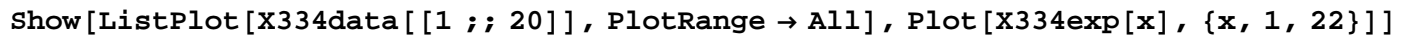

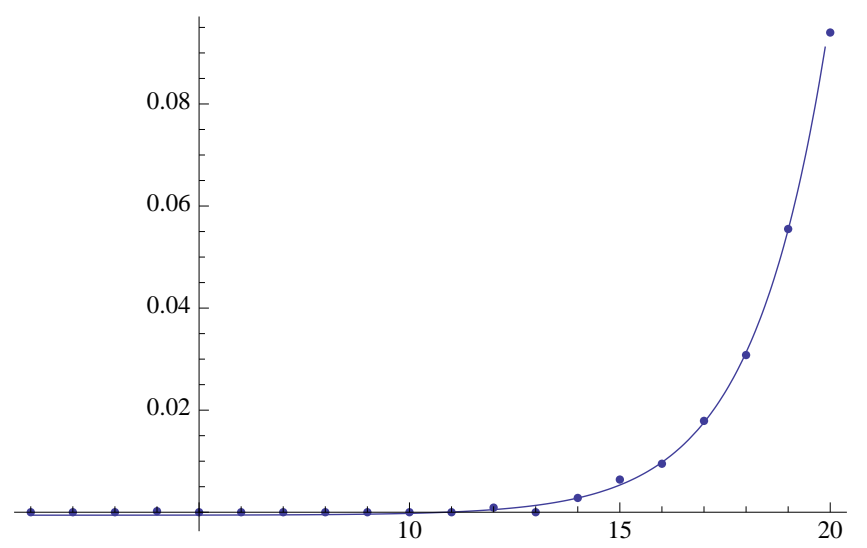

$\mathrm{x} 341 \mathrm{exp}=$

NonlinearModelFit $\left[\mathrm{X} 341\right.$ data $[[2 ; ; 19]], b+d 0 * a^{\wedge} \mathbf{x},\{b, d 0, a\}, \mathbf{x}$, MaxIterations $\left.\rightarrow 1000\right]$

FittedModel $\left[0.000197982+5.11667 \times 10^{-7} \ll 19 \gg^{x}\right]$

x341exp ["BestFit"]

$0.000197982+5.11667 \times 10^{-7} 1.80593^{x}$ 


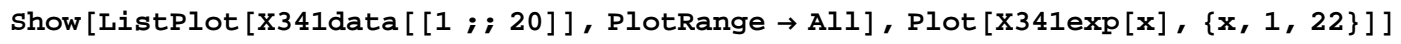

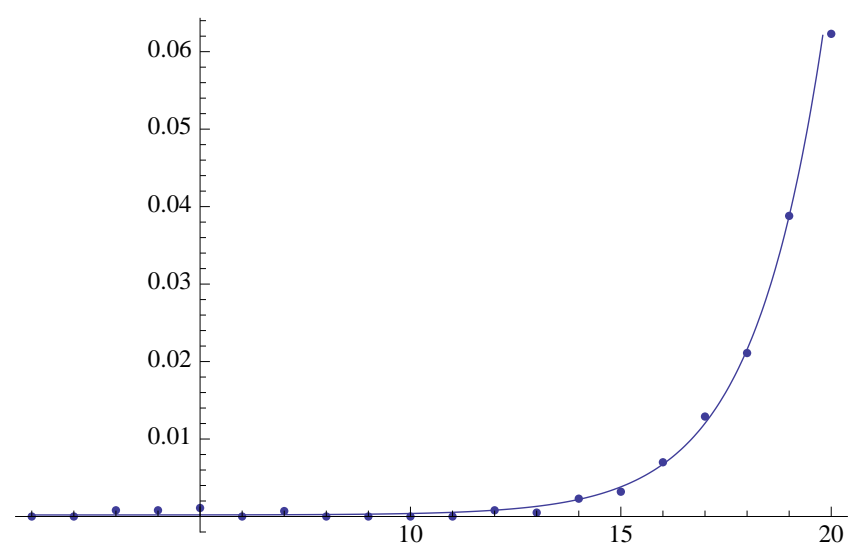

$\mathrm{x} 342 \mathrm{exp}=$

NonlinearModelFit [x342data $[[2 ; ; 19]], b+d 0 * a \wedge x,\{b, d 0, a\}, \mathbf{x}$, MaxIterations $\rightarrow 1000]$

FittedModel $\left[-0.000130338+8.42584 \times 10^{-7} \ll 19 \gg^{x}\right]$

x342exp ["BestFit"]

$-0.000130338+8.42584 \times 10^{-7} 1.77362^{x}$

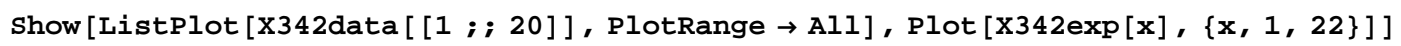

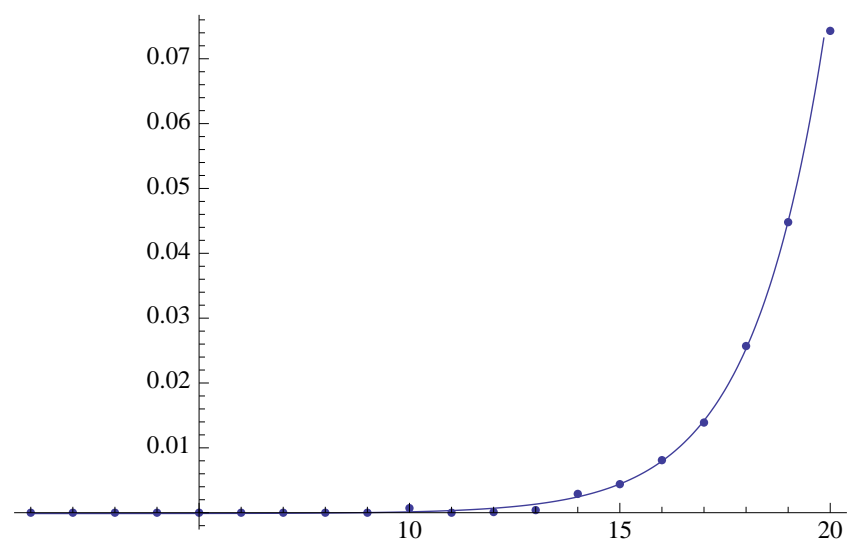

$\mathrm{x} 343 \mathrm{exp}=$

NonlinearModelFit [x343data $[2 ; ; 19]], b+d 0 * a \wedge x,\{b, d 0, a\}, x$, MaxIterations $\rightarrow 1000]$

FittedModel $\left[-0.000264434+1.40858 \times 10^{-6} \ll 19 \gg^{x}\right]$

X343exp ["BestFit"]

$-0.000264434+1.40858 \times 10^{-6} 1.71202^{x}$ 
Show $[$ ListPlot $[\mathrm{X} 343$ data $[1 ; ; 20]], \mathrm{PlotRange} \rightarrow \mathrm{All}], \mathrm{Plot}[\mathrm{X} 343 \exp [\mathrm{x}],\{\mathrm{x}, 1,22\}]$

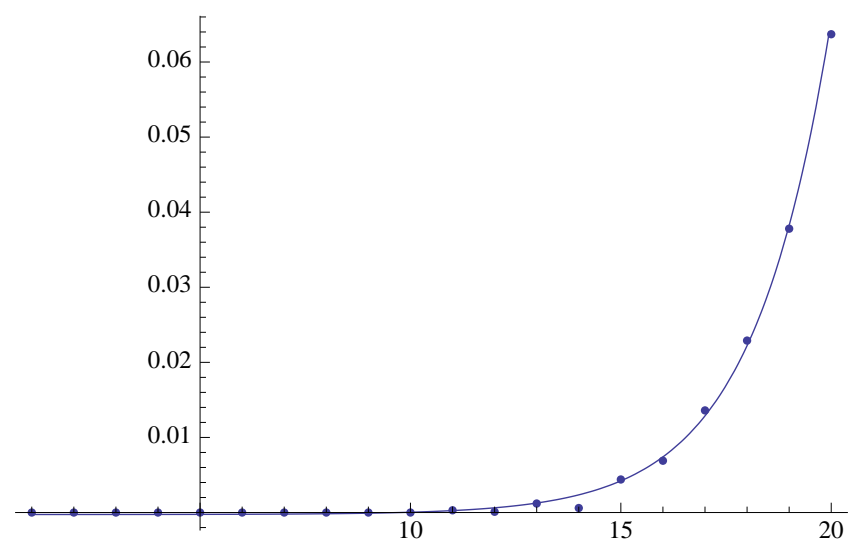

$\mathrm{X} 344 \mathrm{exp}=$

NonlinearModelFit [x344data $[2 ; ; 19]], b+d 0 * a^{\wedge} \mathbf{x},\{b, d 0, a\}, \mathbf{x}$, MaxIterations $\left.\rightarrow 1000\right]$

FittedModel $\left[-0.000290228+2.38968 \times 10^{-6} \ll 18 \gg^{x}\right]$

X344exp [ BestFit"]

$-0.000290228+2.38968 \times 10^{-6} 1.66801^{x}$

Show $[$ ListPlot $[X 344$ data $[1 ; ; 20]], P l o t R a n g e \rightarrow A l l], P 1 o t[X 344 \exp [x],\{x, 1,22\}]]$

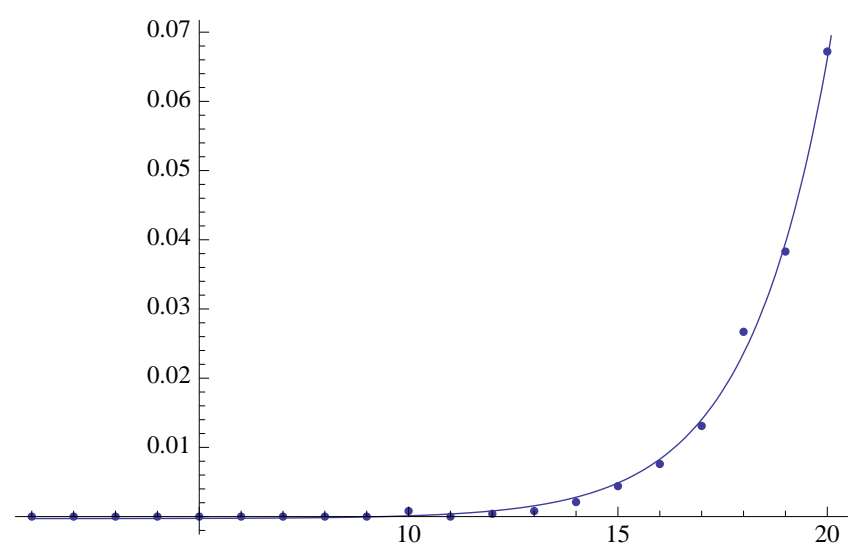

X351exp $=$

NonlinearModelFit [X351data $\left.[[2 ; ; 19]], b+d 0 * a^{\wedge} \mathbf{x},\{b, d 0, a\}, \mathbf{x}, \operatorname{MaxIterations} \rightarrow 1000\right]$

FittedModel $\left[-0.000056511+9.06115 \times 10^{-7} \ll 19 \gg^{x}\right]$

x351exp ["BestFit"]

$-0.000056511+9.06115 \times 10^{-7} 1.7564^{x}$ 
Show [ListPlot $[\mathrm{X} 351$ data $[1 ; ; 20]], \mathrm{PlotRange} \rightarrow \mathrm{All}], \mathrm{Plot}[\mathrm{X} 351 \exp [\mathbf{x}],\{\mathbf{x}, 1,22\}]]$

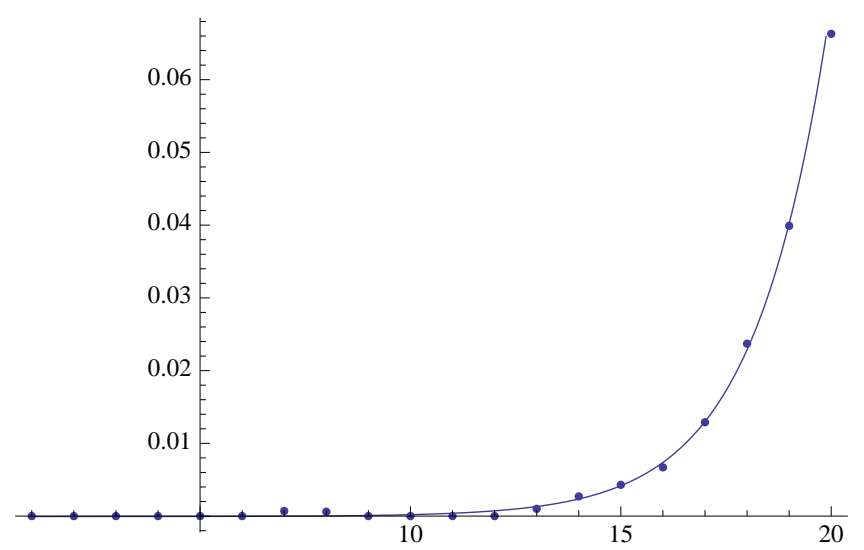

$\mathrm{x} 352 \mathrm{exp}=$

NonlinearModelFit [x352data $[2 ; ; 19]], b+d 0 * a \wedge x,\{b, d 0, a\}, \mathbf{x}$, MaxIterations $\rightarrow 1000]$

FittedModel $\left[-0.0000753951+4.33935 \times 10^{-7} \ll 19 \gg^{x}\right]$

X352exp ["BestFit"]

$-0.0000753951+4.33935 \times 10^{-7} 1.84402^{x}$

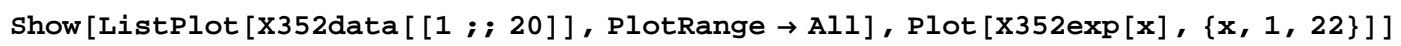

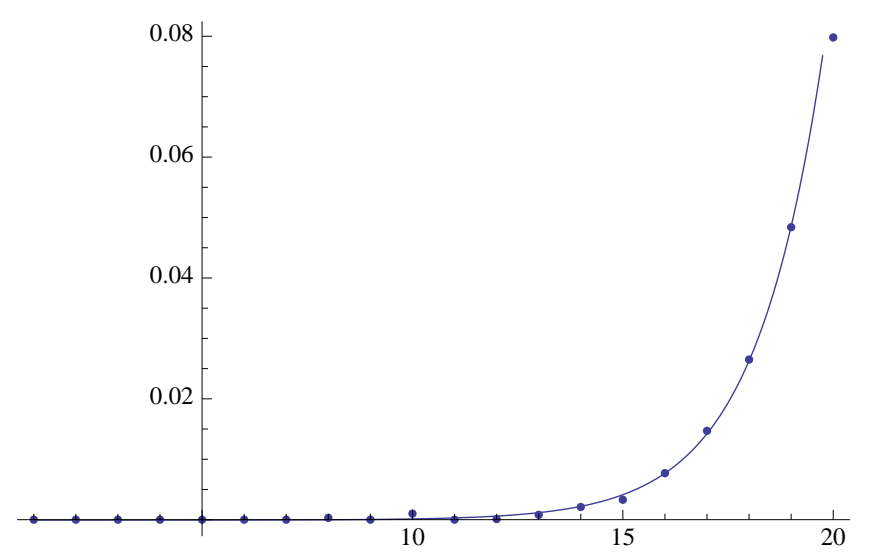

$\mathrm{x} 353 \mathrm{exp}=$

NonlinearModelFit [x353data $[[2 ; ; 19]], b+d 0 * a \wedge x,\{b, d 0, a\}, \mathbf{x}$, MaxIterations $\rightarrow 1000]$

FittedModel $\left[0.0000138645+5.21369 \times 10^{-7} \ll 18 \gg^{x}\right]$

X353exp ["BestFit"]

$0.0000138645+5.21369 \times 10^{-7} 1.82324^{x}$ 
Show $[$ ListPlot $[X 353$ data $[1 ; ; 20]], P l o t R a n g e \rightarrow A l l], P 1 o t[X 353 \exp [\mathbf{x}],\{\mathbf{x}, 1,22\}]]$

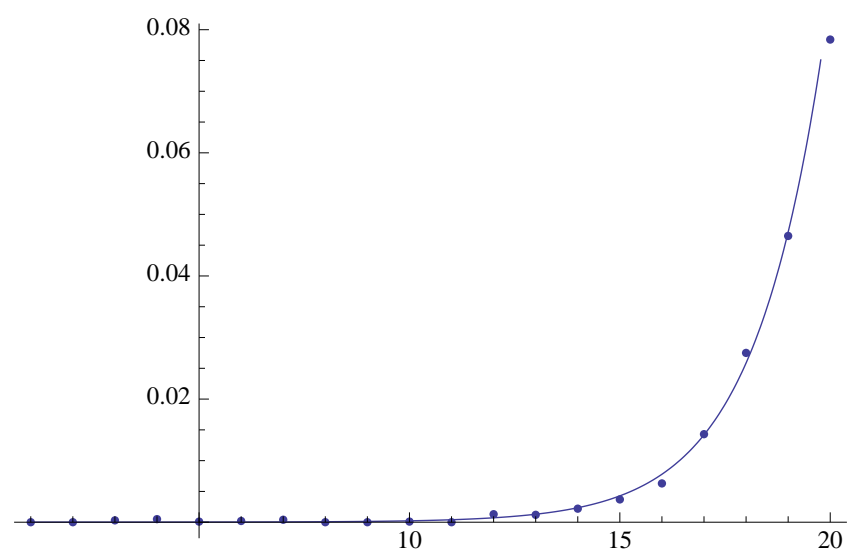

$\mathrm{x} 354 \mathrm{exp}=$

NonlinearModelFit $[x 354$ data $[5 ; ; 20]], b+d 0 * a^{\wedge} \mathbf{x},\{b, d 0, a\}, \mathbf{x}$, MaxIterations $\left.\rightarrow 1000\right]$

FittedModel $\left[-0.00024931+1.32729 \times 10^{-6} \ll 19 \gg^{x}\right]$

X354exp ["BestFit"]

$-0.00024931+1.32729 \times 10^{-6} 1.75395^{x}$

Show $[$ ListPlot $[\mathrm{X} 354$ data $[[1 ; ; 20]], \mathrm{PlotRange} \rightarrow \mathrm{All}], \mathrm{Plot}[\mathrm{X} 354 \exp [\mathbf{x}],\{\mathbf{x}, 1,22\}]]$

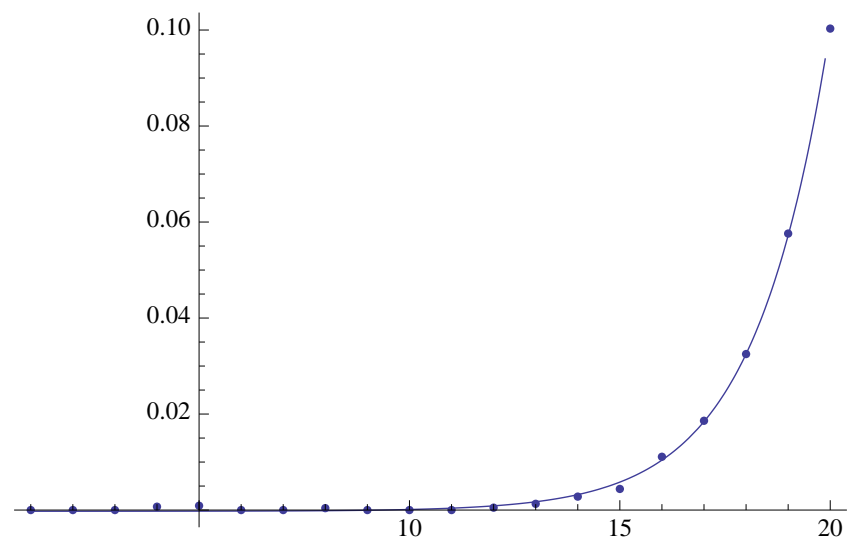

$\mathrm{x} 411 \mathrm{exp}=$

NonlinearModelFit $[\mathbf{x} 411$ data $[13 ; ; 24]], b+d 0 * a^{\wedge} \mathbf{x},\{b, d 0, a\}, \mathbf{x}$, MaxIterations $\left.\rightarrow 1000\right]$

FittedModel $\left[-0.00098573+3.4953 \times 10^{-7} \ll 19 \gg^{x}\right]$

X411exp ["BestFit"]

$-0.00098573+3.4953 \times 10^{-7} 1.66586^{x}$ 
Show $[$ ListPlot $[X 411$ data $[1 ; ; 26]], P l o t R a n g e \rightarrow A l l], P l o t[X 411 \exp [x],\{x, 1,28\}]]$

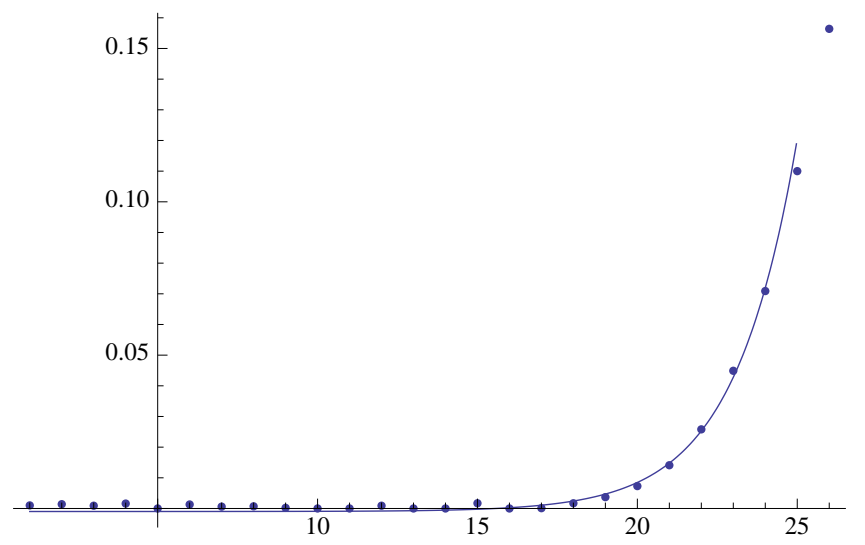

$\mathrm{x} 412 \mathrm{exp}=$

NonlinearModelFit $\left[\mathbf{X} 412\right.$ data $[[13 ; ; 24]], b+d 0 * a^{\wedge} \mathbf{x},\{b, d 0, a\}, \mathbf{x}$, MaxIterations $\left.\rightarrow 1000\right]$

FittedModel $\left[-0.000772384+3.98604 \times 10^{-7} \ll 19 \gg^{x}\right]$

x412exp [ BestFit"]

$-0.000772384+3.98604 \times 10^{-7} 1.65521^{x}$

Show [ListPlot $[\mathrm{X} 412 \operatorname{dat}[[1 ; ; 26]], \mathrm{PlotRange} \rightarrow \mathrm{All}], \mathrm{Plot}[\mathrm{X} 412 \exp [\mathrm{x}],\{\mathrm{x}, 1,29\}]]$

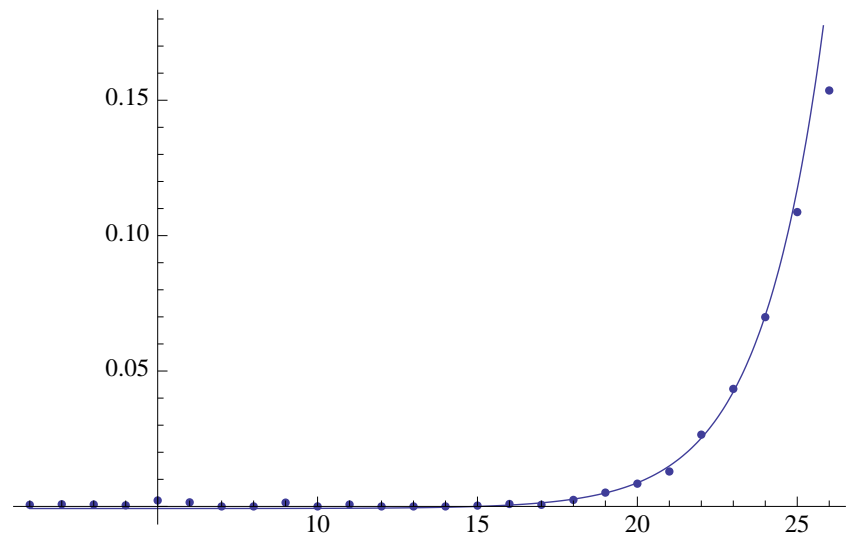

$\mathrm{x} 413 \mathrm{exp}=$

NonlinearModelFit $\left.[\mathrm{X} 413 \mathrm{dat} a[13 ; ; 24]], \mathrm{b}+\mathrm{d} 0 * \mathrm{a}^{\wedge} \mathbf{x},\{\mathrm{b}, \mathrm{d0}, \mathrm{a}\}, \mathbf{x}, \operatorname{MaxIterations} \rightarrow 1000\right]$

FittedModel $\left[-0.000930879+1.87063 \times 10^{-7} \ll 19 \gg^{x}\right]$

X413exp ["BestFit"]

$-0.000930879+1.87063 \times 10^{-7} 1.70983^{x}$ 
Show [ListPlot $[\mathrm{X} 413 \operatorname{dat}[[1 ; ; 24]], \mathrm{PlotRange} \rightarrow \mathrm{All}], \mathrm{Plot}[\mathrm{X} 413 \exp [\mathrm{x}],\{\mathrm{x}, 1,28\}]]$

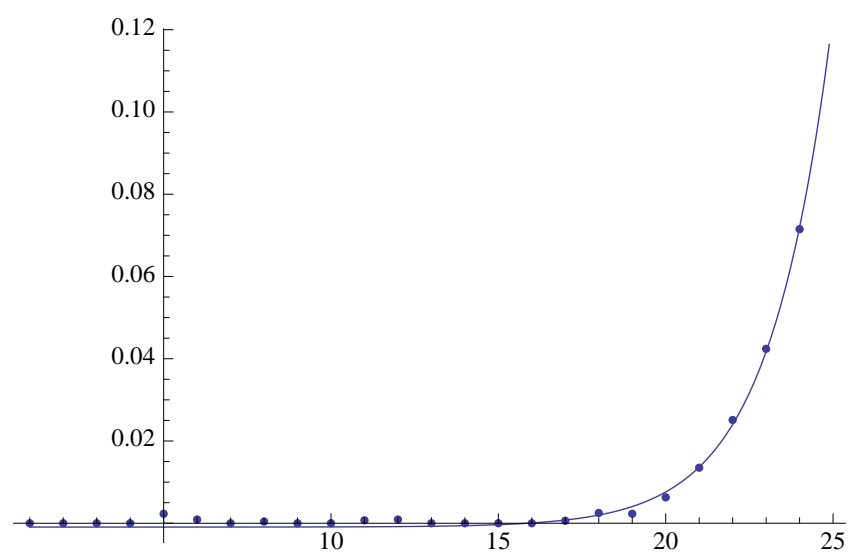

$\mathrm{x} 414 \mathrm{exp}=$

NonlinearModelFit $[\mathbf{x} 414$ data $[13 ; ; 24]], b+d 0 * a^{\wedge} \mathbf{x},\{b, d 0, a\}, \mathbf{x}$, MaxIterations $\left.\rightarrow 1000\right]$

FittedModel $\left[-0.000674251+2.07316 \times 10^{-7} \ll 19>^{x}\right]$

X414exp ["BestFit"]

$-0.000674251+2.07316 \times 10^{-7} 1.70565^{x}$

Show [ListPlot $[\mathrm{X} 414 \operatorname{data}[[1 ; ; 24]], \mathrm{PlotRange} \rightarrow \mathrm{All}], \mathrm{Plot}[\mathrm{X} 414 \exp [\mathrm{x}],\{\mathrm{x}, 1,28\}]]$

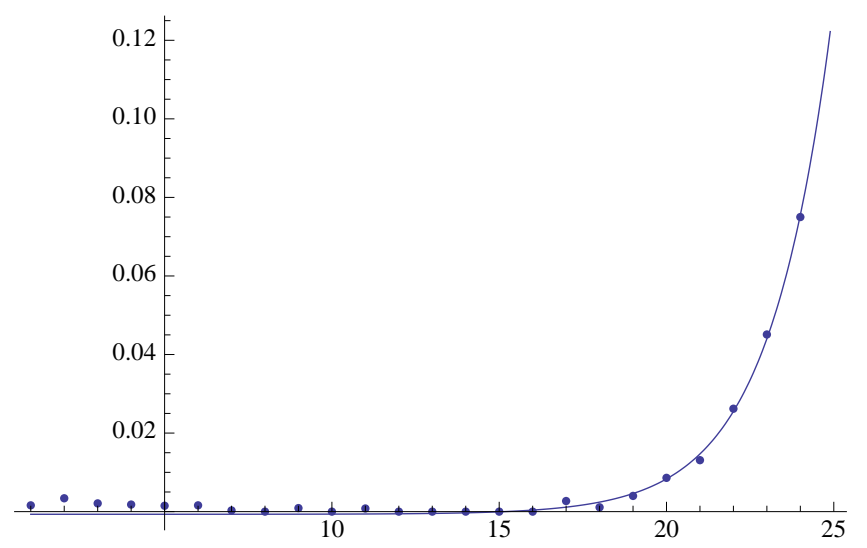

$\mathrm{X} 421 \mathrm{exp}=$

NonlinearModelFit $\left[\mathbf{X} 421\right.$ data $[[13 ; ; 24]], b+d 0 * a^{\wedge} \mathbf{x},\{b, d 0, a\}, \mathbf{x}$, MaxIterations $\left.\rightarrow 1000\right]$

FittedModel $\left[-0.000618286+3.8546 \times 10^{-7} \ll 19 \gg^{x}\right]$

X421exp ["BestFit"]

$-0.000618286+3.8546 \times 10^{-7} 1.66564^{x}$ 
Show [ListPlot $[\mathrm{X} 421$ data $[1 ; ; 23]], \mathrm{PlotRange} \rightarrow \mathrm{All}], \mathrm{Plot}[\mathrm{X} 421 \exp [\mathbf{x}],\{\mathbf{x}, 1,26\}]]$

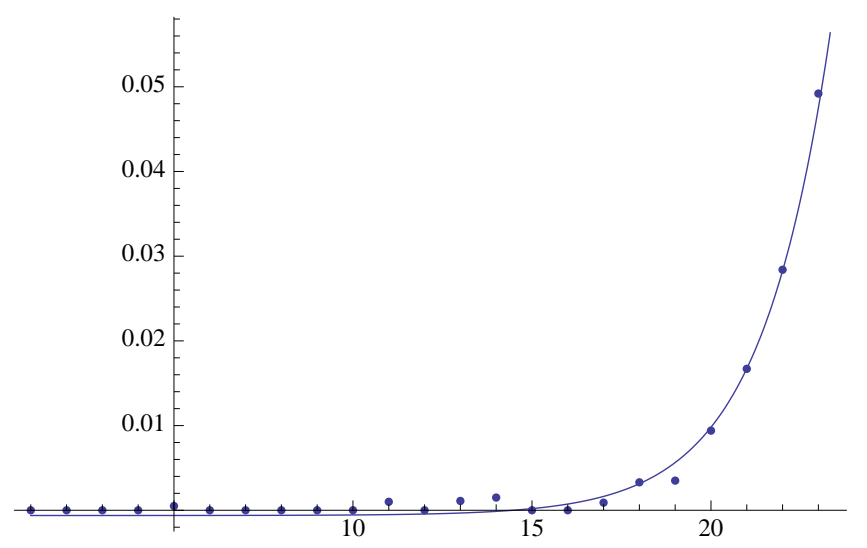

$\mathrm{X422 \operatorname {exp }}=$

NonlinearModelFit $[\mathbf{X} 422$ data $[10 ; ; 23]], b+d 0 * a \wedge x,\{b, d 0, a\}, \mathbf{x}, \operatorname{MaxIterations} \rightarrow 1000]$

FittedModel $\left[-0.000032854+1.83662 \times 10^{-7} \ll 19 \gg^{x}\right]$

$\mathrm{x} 422 \exp [$ "BestFit"]

$-0.000032854+1.83662 \times 10^{-7} 1.73312^{\mathrm{x}}$

Show $[$ ListPlot $[\mathrm{X} 422 \operatorname{dat} a[1 ; ; 23]], \mathrm{PlotRange} \rightarrow \mathrm{All}], \mathrm{Plot}[\mathrm{X} 422 \exp [\mathrm{x}],\{\mathbf{x}, 1,26\}]]$

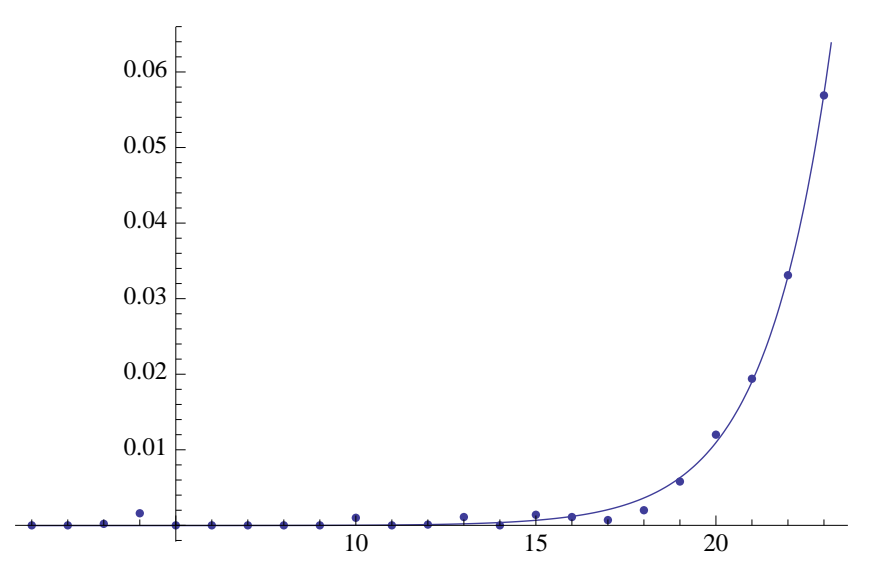

$\mathrm{X} 423 \mathrm{exp}=$

NonlinearModelFit [X423data $[12 ; ; 25]], b+d 0 * a \wedge x,\{b, d 0, a\}, \mathbf{x}, \operatorname{MaxIterations} \rightarrow 1000]$

FittedModel $\left[-0.0013683+1.28066 \times 10^{-6} \ll 19 \gg^{x}\right]$

X423exp ["BestFit"]

$-0.0013683+1.28066 \times 10^{-6} 1.58555^{x}$ 
Show [ListPlot $[\mathrm{X} 423 \operatorname{dat}[[1 ; ; 23]], \mathrm{PlotRange} \rightarrow \mathrm{All}], \mathrm{Plot}[\mathrm{X} 423 \exp [\mathbf{x}],\{\mathbf{x}, 1,26\}]]$

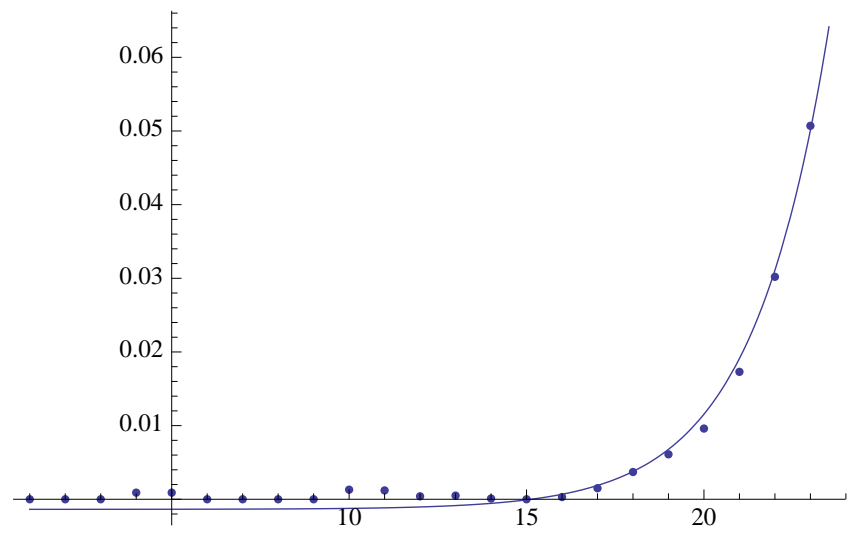

$\mathrm{X} 424 \mathrm{exp}=$

NonlinearModelFit $[\mathbf{X} 44$ data $[12 ; ; 25]], b+d 0 * a^{\wedge} \mathbf{x},\{b, d 0, a\}, \mathbf{x}$, MaxIterations $\left.\rightarrow 1000\right]$

FittedModel $\left[-0.000183795+9.28537 \times 10^{-7} \ll 19 \gg^{x}\right]$

X424exp ["BestFit"]

$-0.000183795+9.28537 \times 10^{-7} 1.60154^{\mathrm{x}}$

Show [ListPlot $[\mathrm{X} 424$ data $[1 ; ; 25]], \mathrm{PlotRange} \rightarrow \mathrm{All}], \mathrm{Plot}[\mathrm{X} 424 \exp [\mathrm{x}],\{\mathrm{x}, 1,28\}]]$

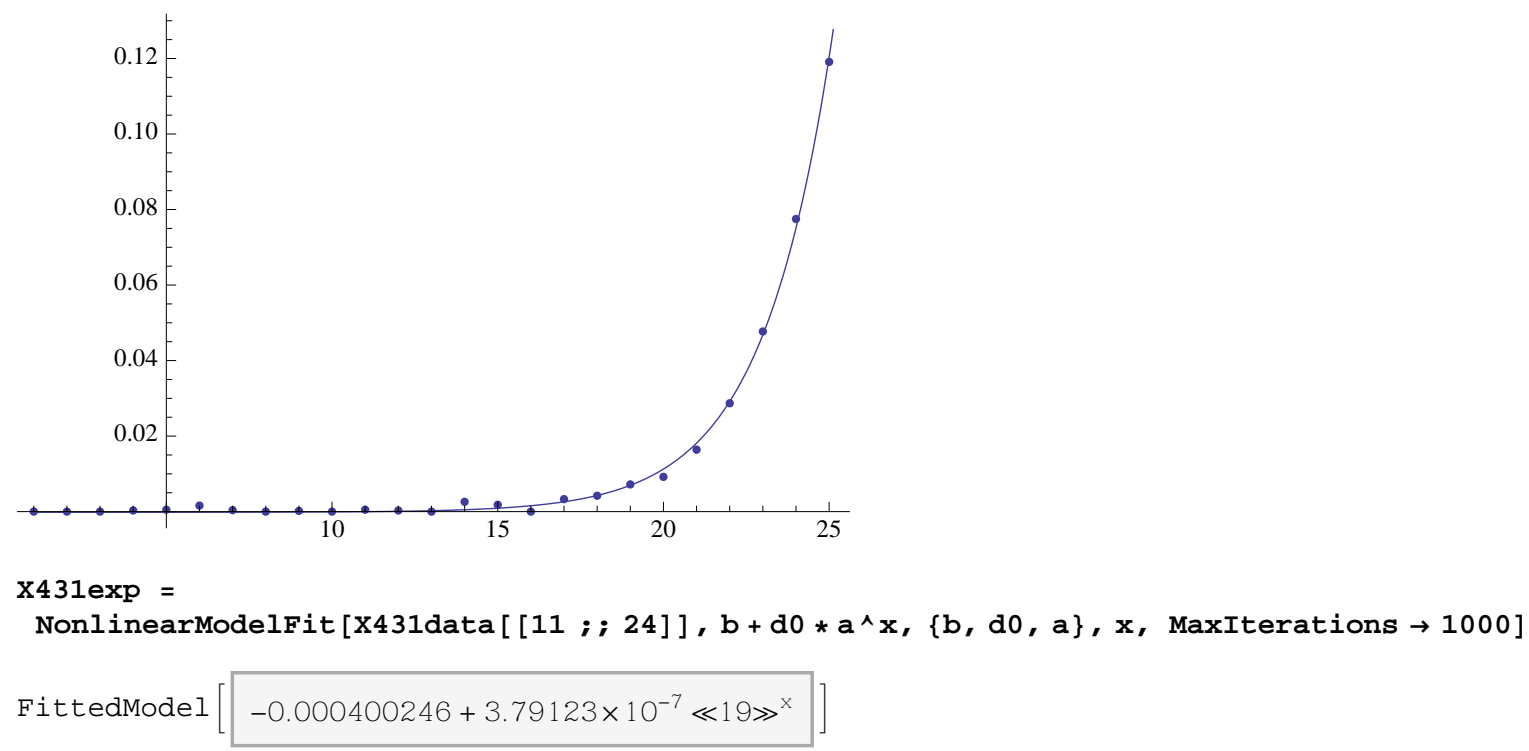

X431exp ["BestFit"]

$-0.000400246+3.79123 \times 10^{-7} 1.68011^{x}$ 
Show [ListPlot $[\mathrm{X} 431$ data $[1 ; ; 23]], \mathrm{PlotRange} \rightarrow \mathrm{All}], \mathrm{Plot}[\mathrm{X} 431 \exp [\mathbf{x}],\{\mathbf{x}, 1,26\}]]$

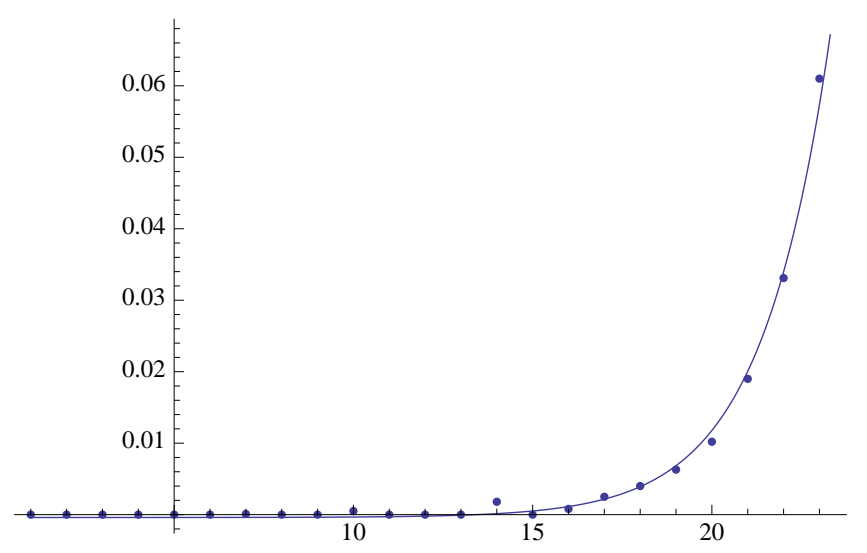

$\mathrm{x} 432 \mathrm{exp}=$

NonlinearModelFit $\left[\mathbf{X} 432\right.$ data $[[11 ; ; 24]], b+d 0 * a^{\wedge} \mathbf{x},\{b, d 0, a\}, \mathbf{x}$, MaxIterations $\left.\rightarrow 1000\right]$

FittedModel $\left[-0.000351816+3.13799 \times 10^{-7} \ll 18 \gg^{x}\right]$

X432exp ["BestFit"]

$-0.000351816+3.13799 \times 10^{-7} 1.69303^{x}$

Show [ListPlot $[\mathrm{X} 432 \operatorname{dat}[[1 ; ; 23]], \mathrm{PlotRange} \rightarrow \mathrm{All}], \mathrm{Plot}[\mathrm{X} 432 \exp [\mathrm{x}],\{\mathrm{x}, 1,26\}]]$

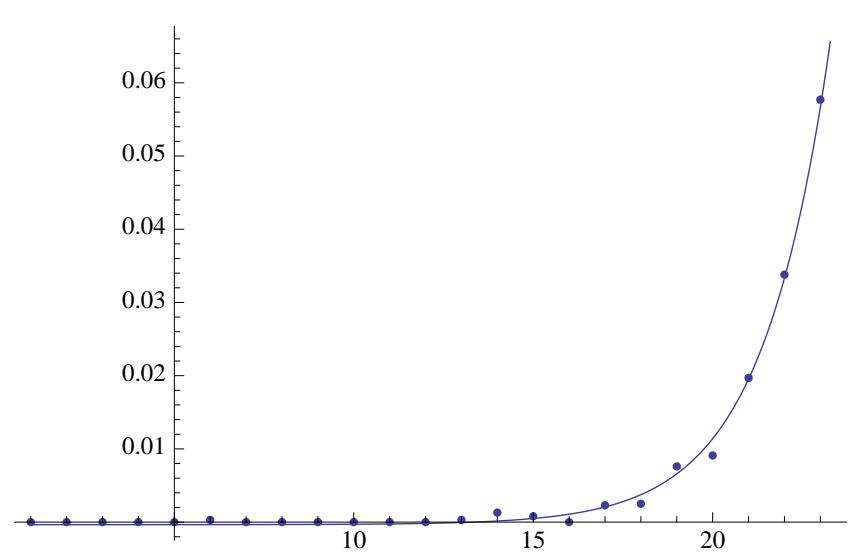

$\mathrm{x} 433 \mathrm{exp}=$

NonlinearModelFit $\left.[\mathrm{X} 433 \mathrm{dat} a[11 ; ; 24]], \mathrm{b}+\mathrm{d} 0 * \mathrm{a}^{\wedge} \mathbf{x},\{b, \mathrm{d0}, \mathrm{a}\}, \mathbf{x}, \operatorname{MaxIterations} \rightarrow 1000\right]$

FittedModel $\left[-0.000280012+3.30429 \times 10^{-7} \ll 18 \gg^{x}\right]$

X433exp ["BestFit"]

$-0.000280012+3.30429 \times 10^{-7} 1.68116^{x}$ 
Show [ListPlot $[\mathrm{X} 433$ data $[1 ; ; 23]], \mathrm{PlotRange} \rightarrow \mathrm{All}], \mathrm{Plot}[\mathrm{X} 433 \exp [\mathrm{x}],\{\mathrm{x}, 1,26\}]]$

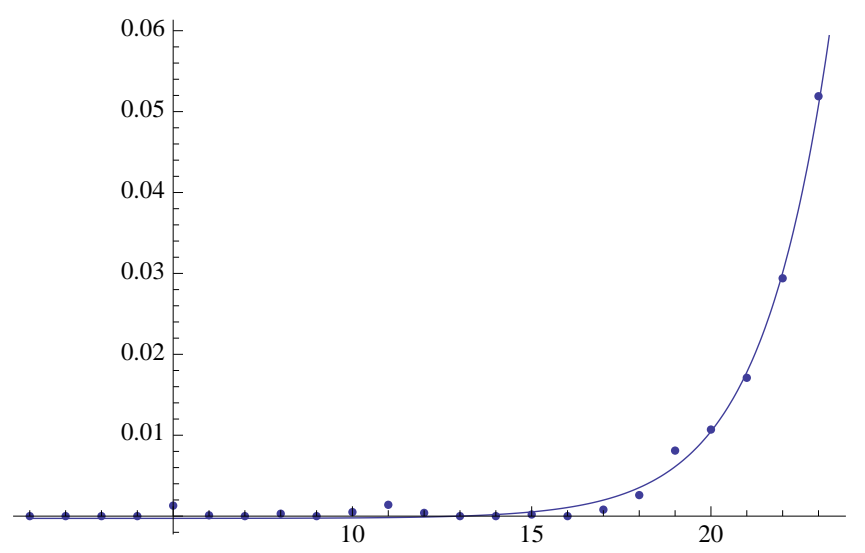

$\mathrm{X} 434 \mathrm{exp}=$

NonlinearModelFit $\left[\mathbf{X} 434\right.$ data $[[11 ; ; 24]], b+d 0 * a^{\wedge} \mathbf{x},\{b, d 0, a\}, \mathbf{x}$, MaxIterations $\left.\rightarrow 1000\right]$

FittedModel $\left[-0.0000122649+3.10098 \times 10^{-7} \ll 19 \gg^{x}\right]$

X434exp ["BestFit"]

$-0.0000122649+3.10098 \times 10^{-7} 1.68435^{x}$

Show [ListPlot $[\mathrm{X} 434$ data $[[1 ; 23]], \mathrm{PlotRange} \rightarrow \mathrm{All}], \mathrm{Plot}[\mathrm{X} 434 \exp [\mathrm{x}],\{\mathrm{x}, 1,26\}]]$

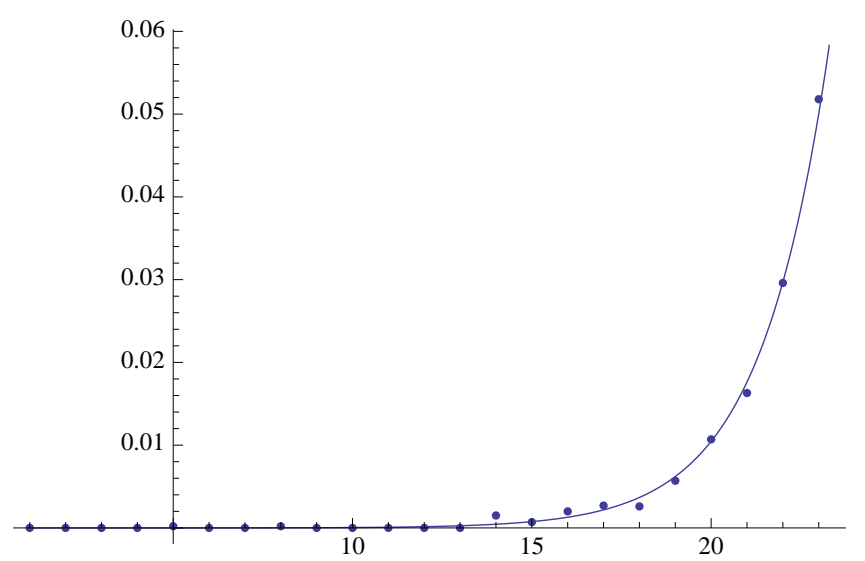

$\mathrm{x} 441 \mathrm{exp}=$

NonlinearModelFit $[\mathbf{x} 441$ data $[13 ; ; 24]], b+d 0 * a^{\wedge} \mathbf{x},\{b, d 0, a\}, \mathbf{x}$, MaxIterations $\left.\rightarrow 1000\right]$

FittedModel $\left[-0.00030012+3.19644 \times 10^{-7} \ll 19 \gg^{x}\right]$

X441exp ["BestFit"]

$-0.00030012+3.19644 \times 10^{-7} 1.66867^{x}$ 
Show [ListPlot $[\mathrm{X} 441$ data $[[1 ; ; 24]], \mathrm{PlotRange} \rightarrow \mathrm{All}], \mathrm{Plot}[\mathrm{X} 441 \exp [\mathrm{x}],\{\mathrm{x}, 1,27\}]]$

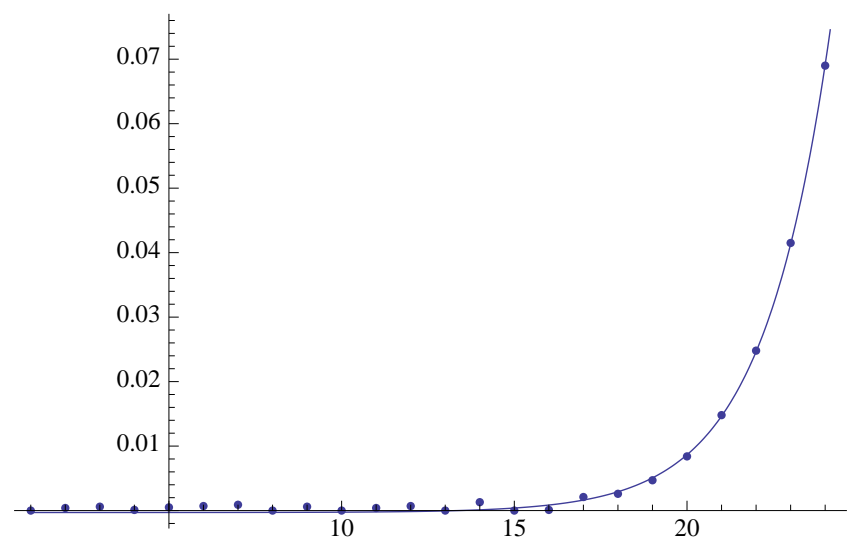

$\mathrm{X} 442 \exp =$

NonlinearModelFit $\left[\mathbf{X} 442\right.$ data $[[13 ; ; 24]], b+d 0 * a^{\wedge} \mathbf{x},\{b, d 0, a\}, \mathbf{x}$, MaxIterations $\left.\rightarrow 1000\right]$

FittedModel $\left[-0.00028773+3.67806 \times 10^{-7} \ll 19 \gg^{x}\right]$

X442exp [ BestFit"]

$-0.00028773+3.67806 \times 10^{-7} 1.6634^{x}$

Show [ListPlot $[\mathrm{X} 442$ data $[[1 ; ; 23]], \mathrm{PlotRange} \rightarrow \mathrm{All}], \mathrm{Plot}[\mathrm{X} 442 \exp [\mathbf{x}],\{\mathbf{x}, 1,26\}]]$

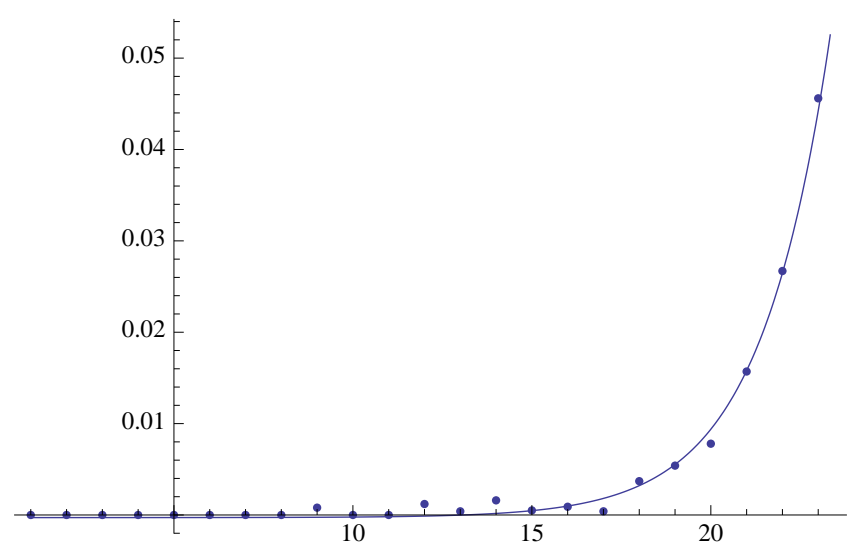

$\mathrm{x} 443 \mathrm{exp}=$

NonlinearModelFit $[\mathrm{X} 443$ data $[[13 ; ; 24]], b+d 0 * a \wedge x,\{b, d 0, a\}, \mathbf{x}$, MaxIterations $\rightarrow 1000]$

FittedModel $\left[0.000177021+2.62147 \times 10^{-7} \ll 19 \gg^{x}\right]$

X443exp ["BestFit"]

$0.000177021+2.62147 \times 10^{-7} 1.67969^{x}$ 
Show [ListPlot $[\mathrm{X} 443 \operatorname{dat}[[1 ; ; 23]], \mathrm{PlotRange} \rightarrow \mathrm{All}], \mathrm{Plot}[\mathrm{X} 443 \exp [\mathrm{x}],\{\mathrm{x}, 1,26\}]]$

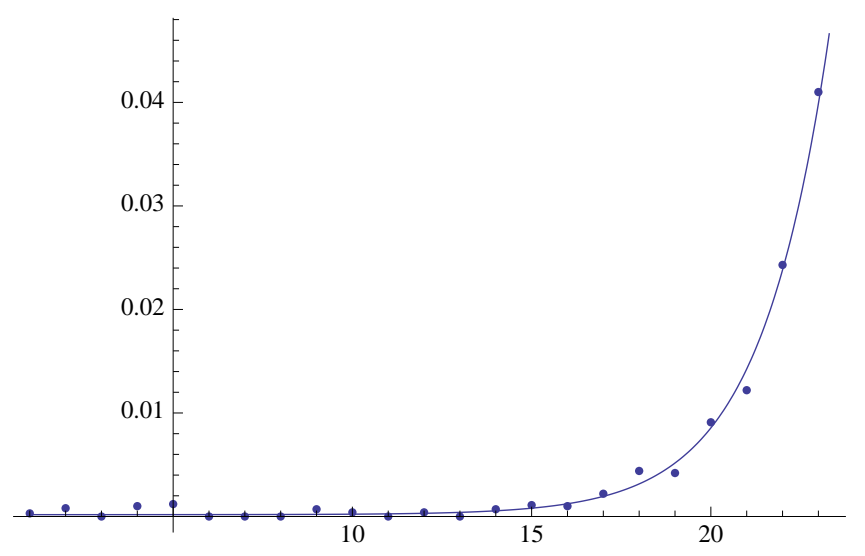

$\mathrm{x} 444 \mathrm{exp}=$

NonlinearModelFit $[\mathbf{X} 44$ data $[13 ; ; 24]], b+d 0 * a^{\wedge} \mathbf{x},\{b, d 0, a\}, \mathbf{x}$, MaxIterations $\left.\rightarrow 1000\right]$

FittedModel $\left[-0.000290316+2.91505 \times 10^{-7} \ll 19 \gg^{x}\right]$

X444exp ["BestFit"]

$-0.000290316+2.91505 \times 10^{-7} 1.67459^{x}$

Show $[$ ListPlot $[X 444$ data $[[1 ; ; 24]], P l o t R a n g e \rightarrow A l l], P l o t[X 444 \exp [x],\{x, 1,27\}]]$

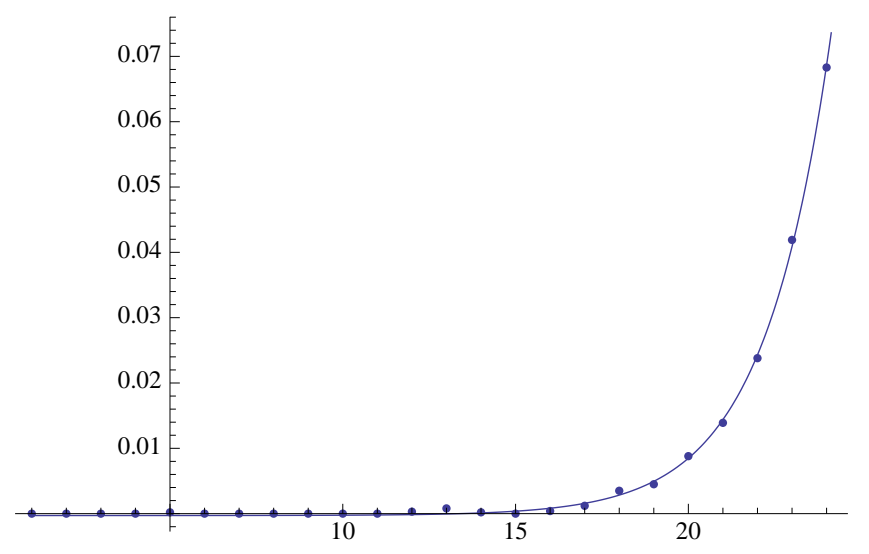

$\mathrm{x} 451 \mathrm{exp}=$

NonlinearModelFit $\left[\mathbf{X} 451\right.$ data $[[13 ; ; 24]], b+d 0 * a^{\wedge} \mathbf{x},\{b, d 0, a\}, \mathbf{x}$, MaxIterations $\left.\rightarrow 1000\right]$

FittedModel $\left[-0.000971878+5.64705 \times 10^{-7} \ll 19 \gg^{x}\right]$

X451exp ["BestFit"]

$-0.000971878+5.64705 \times 10^{-7} 1.63888^{x}$ 
Show [ListPlot $[\mathrm{X} 451$ data $[[1 ; ; 24]], \mathrm{PlotRange} \rightarrow \mathrm{All}], \mathrm{Plot}[\mathrm{X} 451 \exp [\mathrm{x}],\{\mathbf{x}, 1,27\}]]$

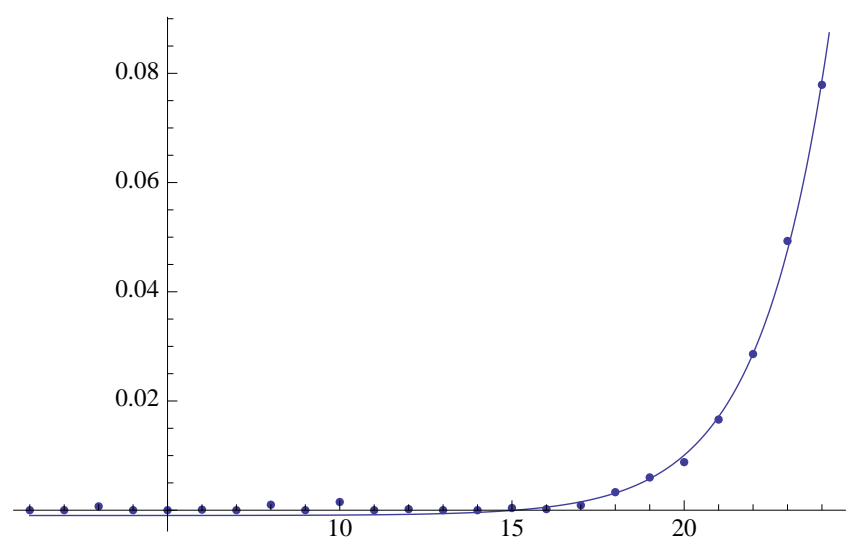

$\mathrm{X} 452 \mathrm{exp}=$

NonlinearModelFit $[\mathbf{X} 452$ data $[11 ; ; 24]], b+d 0 * a \wedge x,\{b, d 0, a\}, \mathbf{x}, \operatorname{MaxIterations} \rightarrow 1000]$

FittedModel $\left[-0.000454658+3.80166 \times 10^{-7} \ll 18 \gg^{x}\right]$

X452exp ["BestFit"]

$-0.000454658+3.80166 \times 10^{-7} 1.6833^{x}$

Show $[$ ListPlot $[\mathrm{X} 452$ data $[[1 ; ; 24]], \mathrm{PlotRange} \rightarrow \mathrm{All}], \mathrm{Plot}[\mathrm{X} 452 \exp [\mathrm{x}],\{\mathrm{x}, 1,27\}]]$

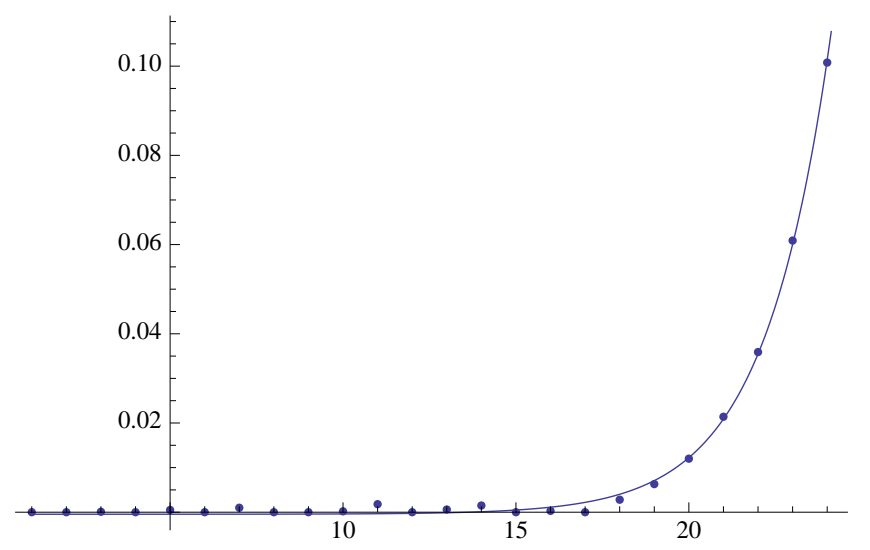

$\mathrm{X} 453 \mathrm{exp}=$

NonlinearModelFit $[\mathrm{X} 453$ data $[11 ; ; 24]], b+d 0 * a^{\wedge} \mathbf{x},\{b, d 0, a\}, \mathbf{x}$, MaxIterations $\left.\rightarrow 1000\right]$

FittedModel $\left[-0.000238962+4.96792 \times 10^{-7} \ll 19 \gg^{x}\right]$

x453exp ["BestFit"]

$-0.000238962+4.96792 \times 10^{-7} 1.65712^{x}$ 
Show [ListPlot $[\mathrm{X} 453 \operatorname{dat}[[1 ; ; 24]], \mathrm{PlotRange} \rightarrow \mathrm{All}], \mathrm{Plot}[\mathrm{X} 453 \exp [\mathrm{x}],\{\mathrm{x}, 1,27\}]]$

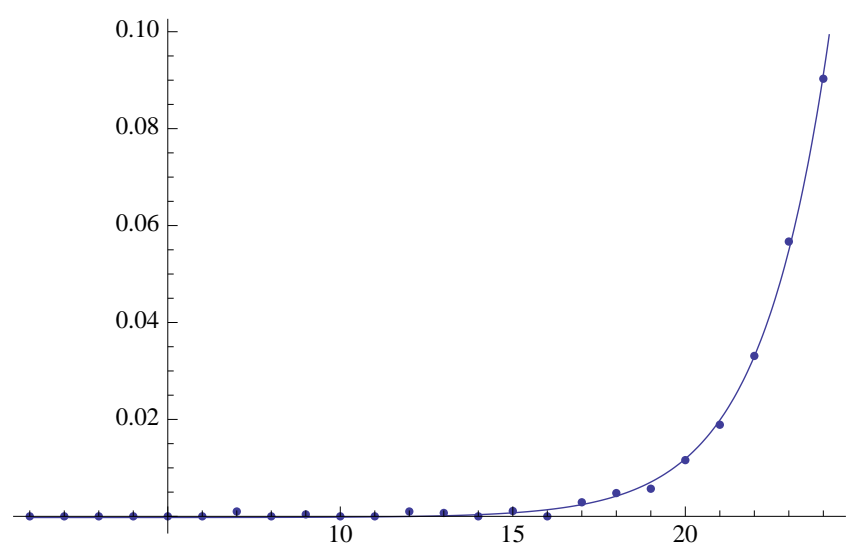

$\mathrm{X} 454 \mathrm{exp}=$

NonlinearModelFit $\left[\mathbf{X} 454\right.$ data $[[13 ; ; 24]], b+d 0 * a^{\wedge} \mathbf{x},\{b, d 0, a\}, \mathbf{x}$, MaxIterations $\left.\rightarrow 1000\right]$

FittedModel $\left[-0.000516062+2.61187 \times 10^{-7} \ll 19 \gg^{x}\right]$

X454exp [ BestFit"]

$-0.000516062+2.61187 \times 10^{-7} 1.69537^{x}$

Show [ListPlot $[\mathrm{X} 454$ data $[[1 ; ; 24]], \mathrm{PlotRange} \rightarrow \mathrm{All}], \mathrm{Plot}[\mathrm{X} 454 \exp [\mathrm{x}],\{\mathrm{x}, 1,27\}]]$

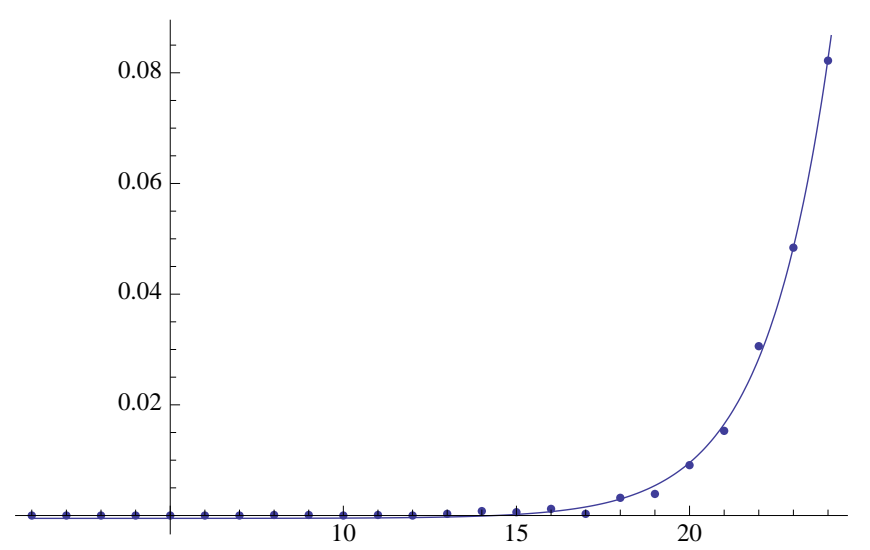

$\mathrm{x} 511 \mathrm{exp}=$

NonlinearModelFit $\left[\mathbf{X} 511\right.$ data $[[15 ; ; 27]], b+d 0 * a^{\wedge} \mathbf{x},\{b, d 0, a\}, \mathbf{x}$, MaxIterations $\left.\rightarrow 1000\right]$

FittedModel $\left[-0.000738987+4.47959 \times 10^{-8} \ll 19 \gg^{x}\right]$

X511exp ["BestFit"]

$-0.000738987+4.47959 \times 10^{-8} 1.71203^{x}$ 
Show [ListPlot $[\mathrm{X} 511$ data $[[1 ; ; 28]], \mathrm{PlotRange} \rightarrow \mathrm{All}], \mathrm{Plot}[\mathrm{X} 511 \exp [\mathbf{x}],\{\mathbf{x}, 1,30\}]]$

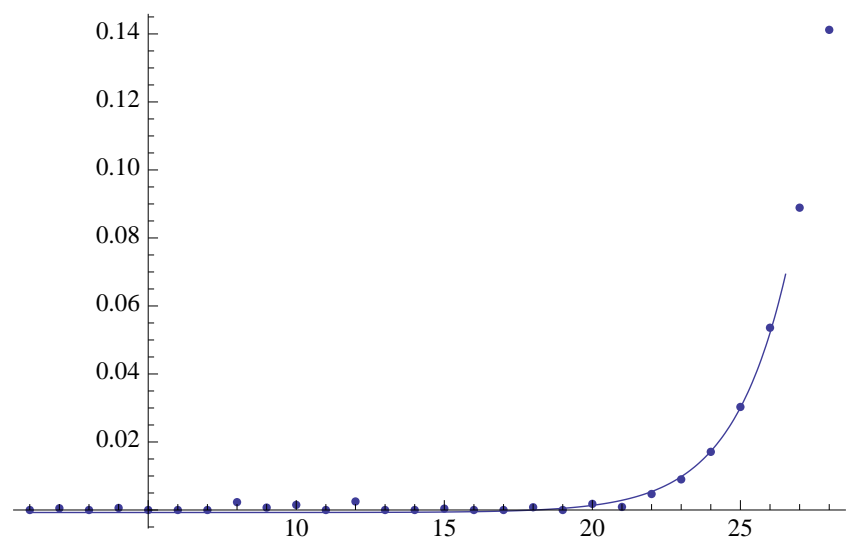

$\mathrm{x} 512 \mathrm{exp}=$

NonlinearModelFit $\left[\mathrm{X} 512\right.$ data $[[15 ; ; 27]], b+d 0 * a^{\wedge} \mathbf{x},\{b, d 0, a\}, \mathbf{x}$, MaxIterations $\left.\rightarrow 1000\right]$

FittedModel $\left[-0.000331286+2.66729 \times 10^{-8} \ll 19 \gg^{x}\right]$

X512exp [ BestFit"]

$-0.000331286+2.66729 \times 10^{-8} 1.74297^{x}$

Show [ListPlot $[\mathrm{X} 512 \operatorname{dat}[[1 ; ; 27]], \mathrm{PlotRange} \rightarrow \mathrm{All}], \mathrm{Plot}[\mathrm{X} 512 \exp [\mathbf{x}],\{\mathbf{x}, 1,30\}]]$

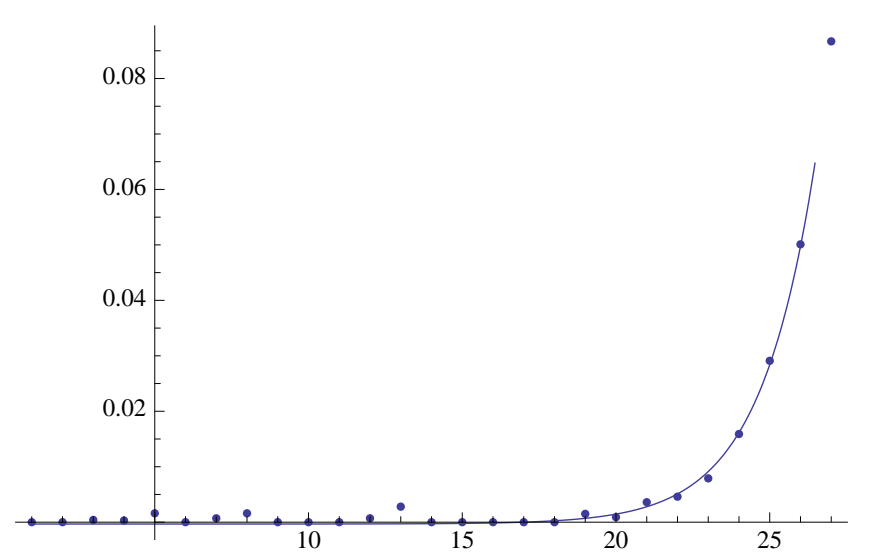

$\mathrm{x} 513 \mathrm{exp}=$

NonlinearModelFit $[\mathrm{X} 513$ data $[[15 ; ; 27]], b+d 0 * a \wedge \mathbf{x},\{b, d 0, a\}, \mathbf{x}, \operatorname{MaxIterations} \rightarrow 1000]$

FittedModel $\left[-0.000301367+3.83936 \times 10^{-8} \ll 19 \gg^{x}\right]$

X513exp ["BestFit"]

$-0.000301367+3.83936 \times 10^{-8} 1.71503^{x}$ 
Show [ListPlot $[\mathrm{X} 513 \operatorname{dat}[[1 ; ; 27]], \mathrm{PlotRange} \rightarrow \mathrm{All}], \mathrm{Plot}[\mathrm{X513exp}[\mathbf{x}],\{\mathbf{x}, 1,31\}]]$

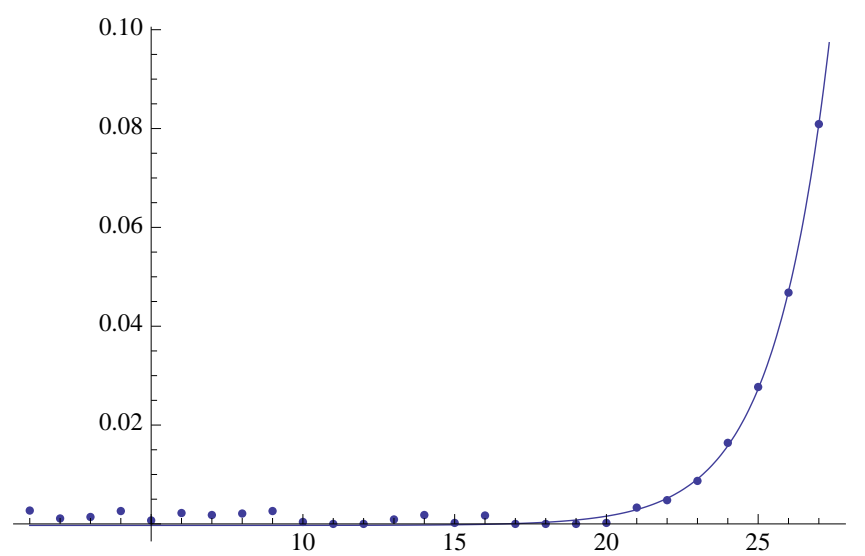

$\mathrm{x} 514 \mathrm{exp}=$

NonlinearModelFit $[\mathrm{x} 514$ data $[15 ; ; 27]], b+d 0 * a^{\wedge} \mathbf{x},\{b, d 0, a\}, \mathbf{x}$, MaxIterations $\left.\rightarrow 1000\right]$

FittedModel $\left[-0.000209407+2.03618 \times 10^{-8} \ll 19 \gg^{x}\right]$

X514exp ["BestFit"]

$-0.000209407+2.03618 \times 10^{-8} 1.74617^{x}$

Show $[$ ListPlot $[\mathrm{X} 514$ data $[[1 ; ; 27]], \mathrm{PlotRange} \rightarrow \mathrm{All}], \mathrm{Plot}[\mathrm{X} 514 \exp [\mathbf{x}],\{\mathbf{x}, 1,30\}]]$

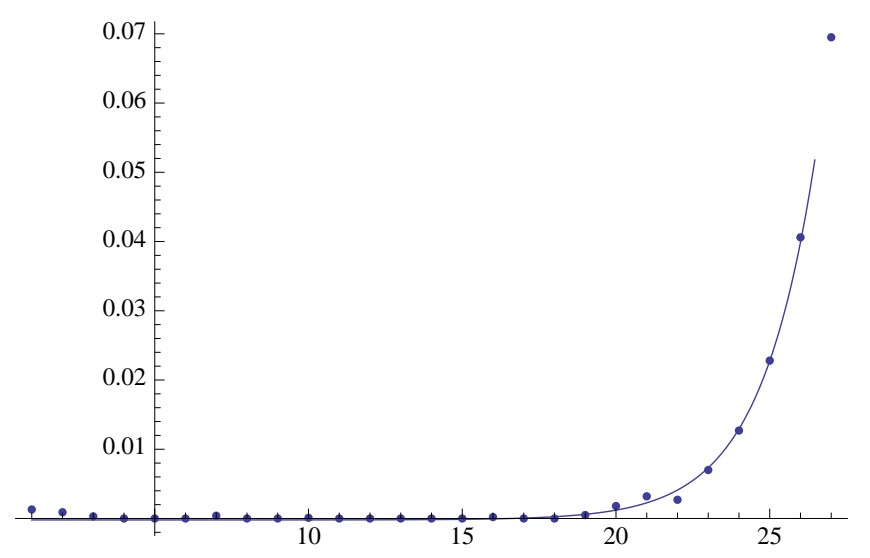

$\mathrm{X} 521 \mathrm{exp}=$

NonlinearModelFit [X521data $[13 ; ; 27]], b+d 0 * a^{\wedge} \mathbf{x},\{b, d 0, a\}, \mathbf{x}$, MaxIterations $\left.\rightarrow 1000\right]$

FittedModel $\left[-0.000285885+4.9818 \times 10^{-8} \ll 18 \gg^{x}\right]$

X521exp ["BestFit"]

$-0.000285885+4.9818 \times 10^{-8} 1.69672^{\mathrm{x}}$ 


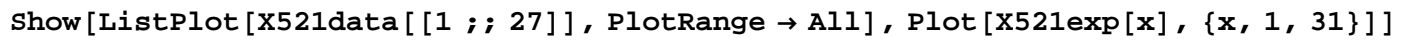

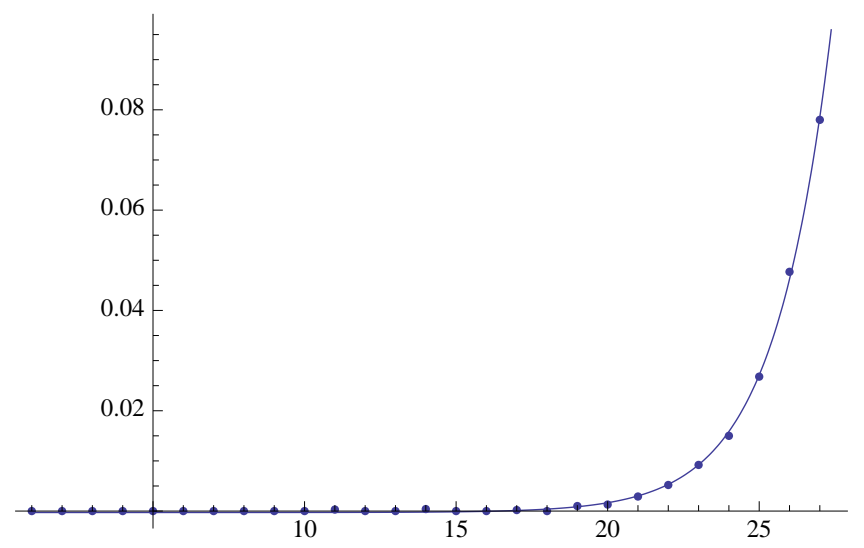

$\mathrm{X} 522 \mathrm{exp}=$

NonlinearModelFit [X522data $[[13 ; ; 27]], b+d 0 * a^{\wedge} \mathbf{x},\{b, d 0, a\}, \mathbf{x}$, MaxIterations $\left.\rightarrow 1000\right]$

FittedModel $\left[-0.000318698+5.26792 \times 10^{-8} \ll 19 \gg^{x}\right]$

X522exp ["BestFit"]

$-0.000318698+5.26792 \times 10^{-8} 1.6907^{x}$

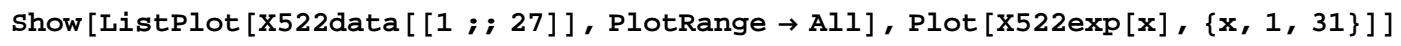

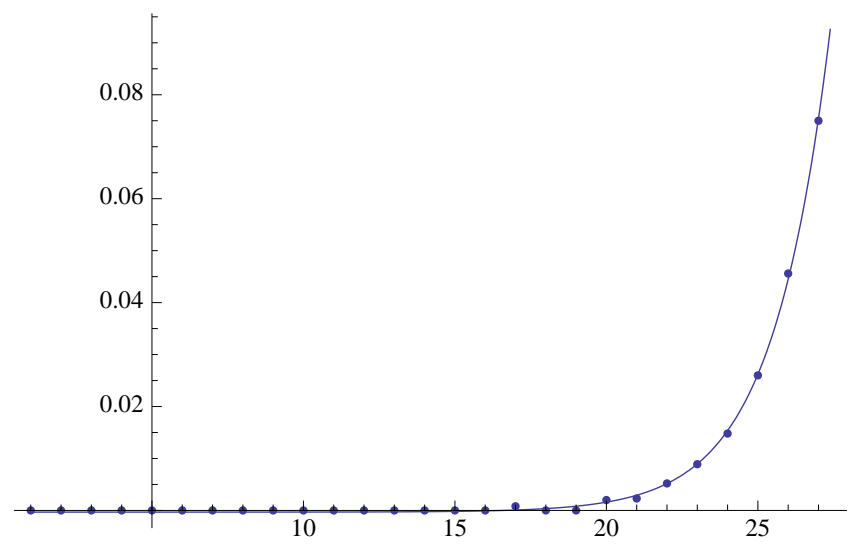

$\mathrm{X} 523 \mathrm{exp}=$

NonlinearModelFit $\left[\mathbf{X} 523\right.$ data $[[13 ; ; 27]], b+d 0 * a^{\wedge} \mathbf{x},\{b, d 0, a\}, \mathbf{x}$, MaxIterations $\left.\rightarrow 1000\right]$

FittedModel $\left[-0.000354803+3.74318 \times 10^{-8} \ll 19 \gg^{x}\right]$

X523exp ["BestFit"]

$-0.000354803+3.74318 \times 10^{-8} 1.716^{x}$ 
Show [ListPlot $[\mathrm{X523data}[[1 ; ; 27]], \mathrm{PlotRange} \rightarrow \mathrm{All}], \mathrm{Plot}[\mathrm{X523exp}[\mathbf{x}],\{\mathbf{x}, 1,31\}]]$

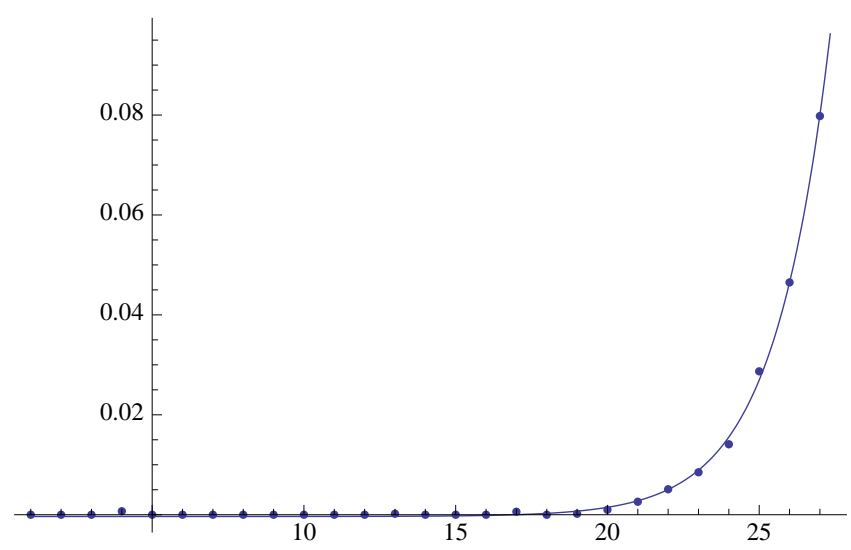

$\mathrm{X} 524 \mathrm{exp}=$

NonlinearModelFit $\left[\mathbf{X} 524\right.$ data $[[13 ; ; 27]], b+d 0 * a^{\wedge} \mathbf{x},\{b, d 0, a\}, \mathbf{x}$, MaxIterations $\left.\rightarrow 1000\right]$

FittedModel $\left[-0.000200681+2.2863 \times 10^{-8} \ll 18 \gg^{x}\right]$

X524exp [ BestFit"]

$-0.000200681+2.2863 \times 10^{-8} 1.7433^{x}$

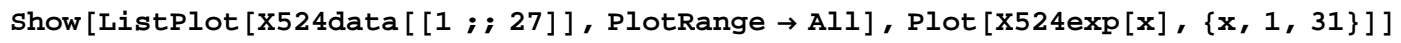

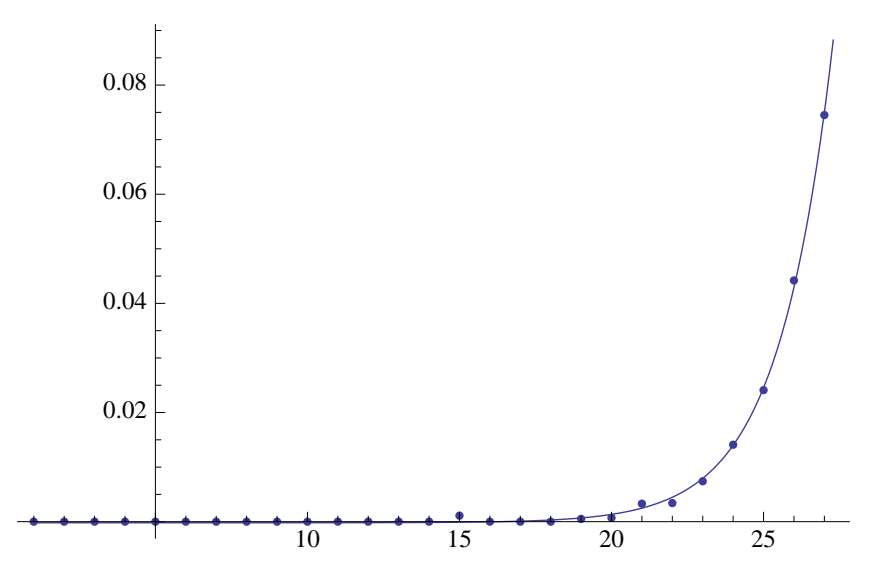

$\mathrm{x} 531 \mathrm{exp}=$

NonlinearModelFit $\left[\mathbf{x} 531\right.$ data $[[13 ; ; 27]], b+d 0 * a^{\wedge} \mathbf{x},\{b, d 0, a\}, \mathbf{x}$, MaxIterations $\left.\rightarrow 1000\right]$

FittedModel $\left[-0.000509783+7.10334 \times 10^{-8} \ll 19 \gg^{x}\right]$

X531exp ["BestFit"]

$-0.000509783+7.10334 \times 10^{-8} 1.67938^{x}$ 


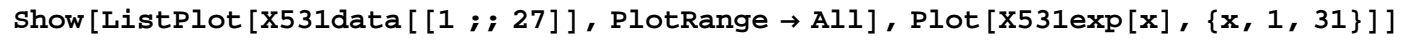

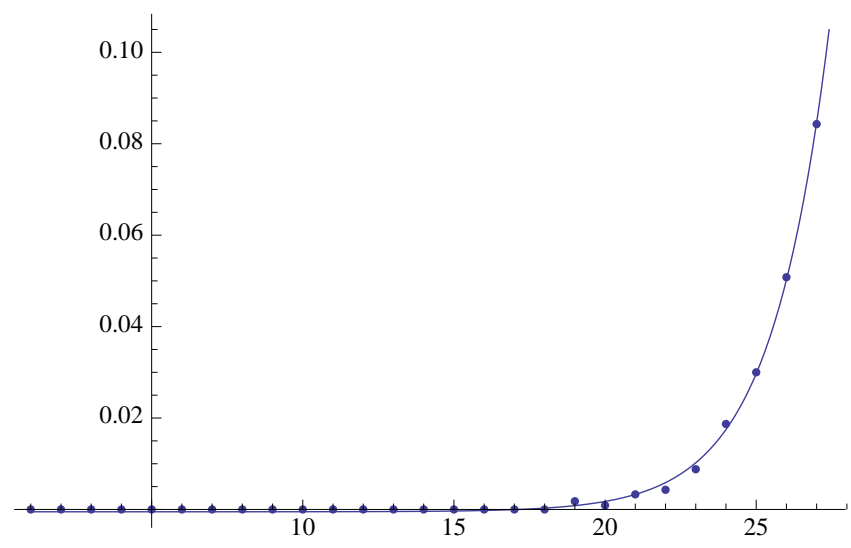

$\mathrm{x} 532 \mathrm{exp}=$

NonlinearModelFit $[\mathbf{X} 532$ data $[13 ; ; 27]], b+d 0 * a \wedge \mathbf{x},\{b, d 0, a\}, \mathbf{x}$, MaxIterations $\rightarrow 1000]$

FittedModel $\left[-0.000262809+4.7395 \times 10^{-8} \ll 18 \gg^{x}\right]$

X532exp ["BestFit"]

$-0.000262809+4.7395 \times 10^{-8} 1.7032^{x}$

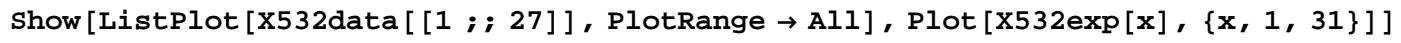

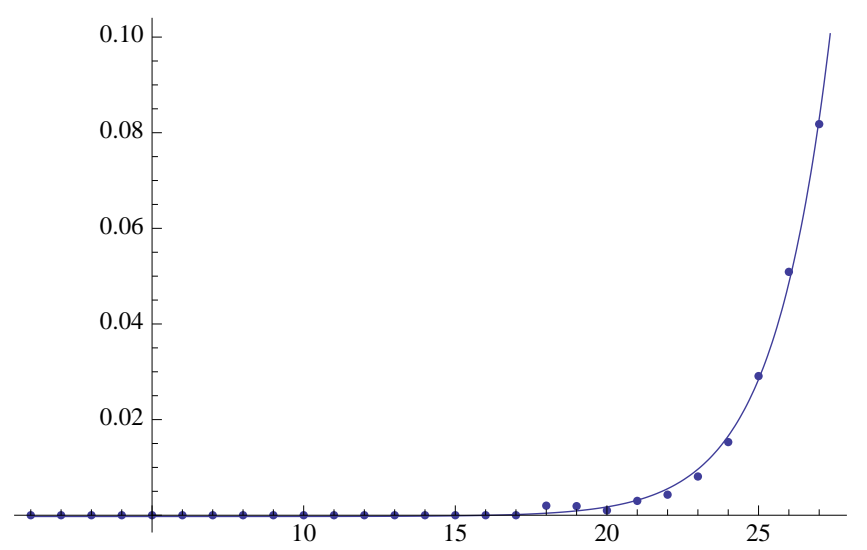

$\mathrm{x} 533 \mathrm{exp}=$

NonlinearModelFit $[\mathbf{x} 533$ data $[13 ; ; 27]], b+d 0 * a \wedge x,\{b, d 0, a\}, \mathbf{x}$, MaxIterations $\rightarrow 1000]$

FittedModel $\left[-0.000370126+3.02444 \times 10^{-8} \ll 19 \gg^{x}\right]$

X533exp ["BestFit"]

$-0.000370126+3.02444 \times 10^{-8} 1.73662^{x}$ 
Show [ListPlot $[\mathrm{X533data}[[1 ; ; 27]], \mathrm{PlotRange} \rightarrow \mathrm{All}], \mathrm{Plot}[\mathrm{X533exp}[\mathbf{x}],\{\mathbf{x}, 1,31\}]]$

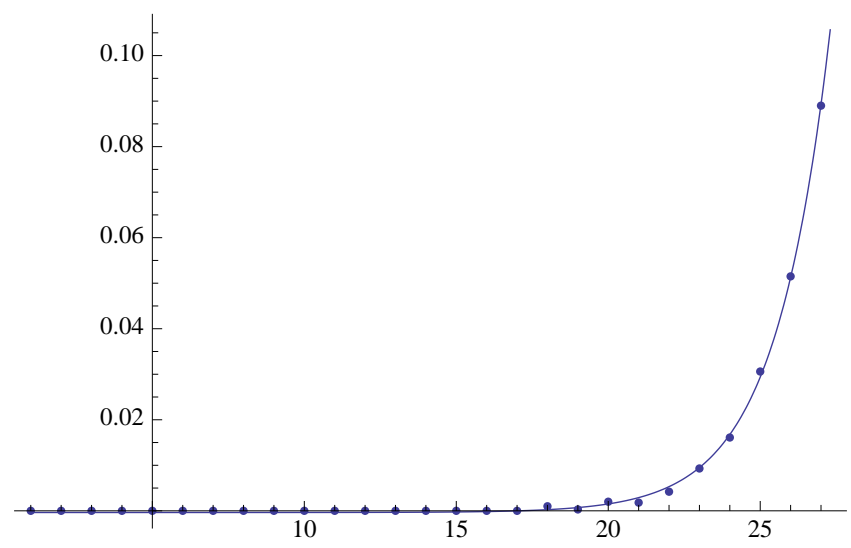

$\mathrm{X} 534 \mathrm{exp}=$

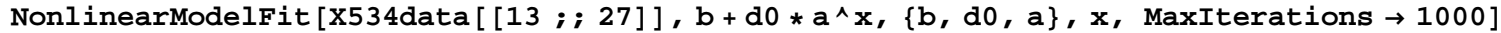

FittedModel $\left[-0.000524581+6.14339 \times 10^{-8} \ll 19 \gg^{\times}\right]$

X534exp [ BestFit"]

$-0.000524581+6.14339 \times 10^{-8} 1.68776^{x}$

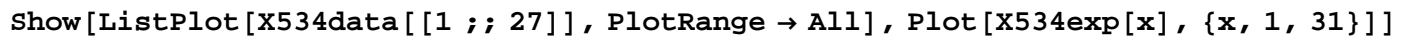

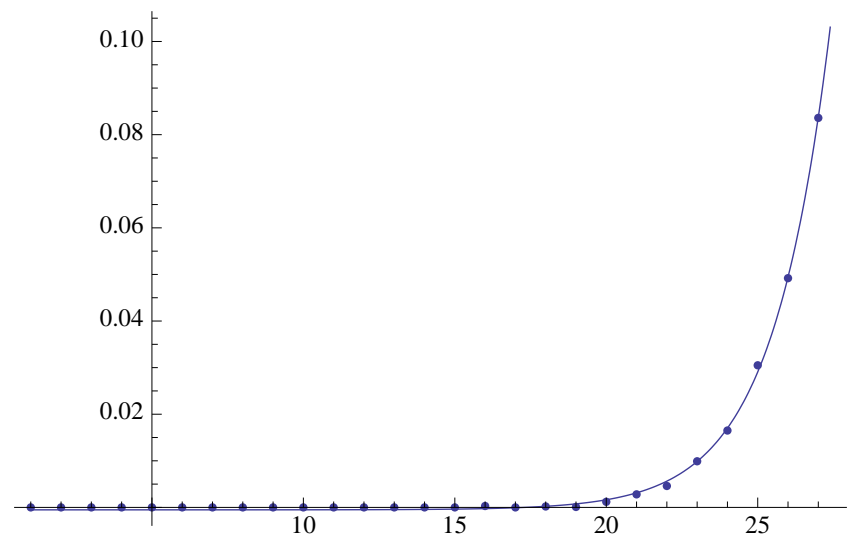

$\mathrm{X} 541 \mathrm{exp}=$

NonlinearModelFit $[\mathrm{X} 541$ data $[[14 ; ; 28]], b+d 0 * a \wedge \mathbf{x},\{b, d 0, a\}, \mathbf{x}$, MaxIterations $\rightarrow 1000]$

FittedModel $\left[-0.00113956+1.33382 \times 10^{-7} \ll 19 \gg^{x}\right]$

X541exp [ BestFit"]

$-0.00113956+1.33382 \times 10^{-7} 1.62869^{x}$ 


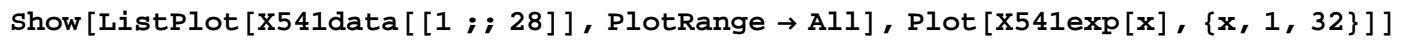

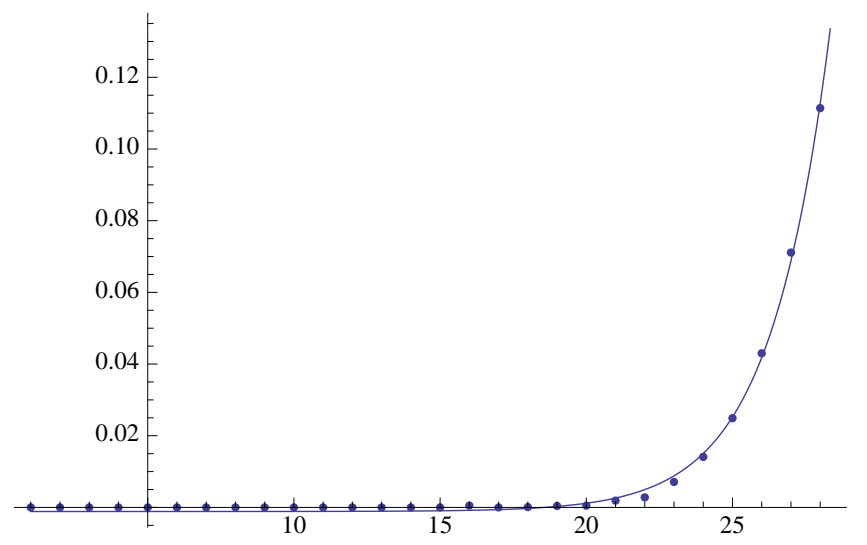

$\mathrm{x} 542 \mathrm{exp}=$

NonlinearModelFit [X542data $[14 ; ; 28]], b+d 0 * a \wedge x,\{b, d 0, a\}, \mathbf{x}, \operatorname{MaxIterations} \rightarrow 1000]$

FittedModel $\left[-0.000649883+4.58214 \times 10^{-8} \ll 16 \gg^{x}\right]$

X542exp [ BestFit"]

$-0.000649883+4.58214 \times 10^{-8} 1.68604^{x}$

Show [ListPlot $[X 542$ data $[1 ; ; 28]], \mathrm{PlotRange} \rightarrow \mathrm{All}], \mathrm{P} 1$ ot $[\mathrm{X542 \operatorname {exp }}[\mathbf{x}],\{\mathbf{x}, 1,32\}]]$

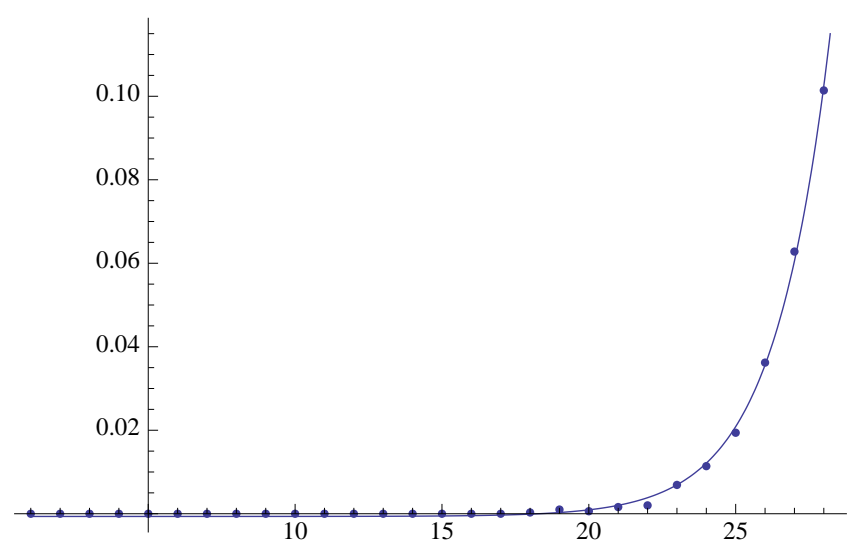

$\mathrm{x} 543 \exp =$

NonlinearModelFit $[\mathrm{X} 543$ data $[13 ; ; 27]], b+d 0 * a \wedge \mathbf{x},\{b, d 0, a\}, \mathbf{x}, \operatorname{MaxIterations} \rightarrow 1000]$

FittedModel $\left[-0.00040915+3.17519 \times 10^{-8} \ll 19 \gg^{x}\right]$

x543exp ["BestFit"]

$-0.00040915+3.17519 \times 10^{-8} 1.72533^{x}$ 
Show [ListPlot $[\mathrm{X543data}[[1 ; ; 27]], \mathrm{PlotRange} \rightarrow \mathrm{All}], \mathrm{Plot}[\mathrm{X543exp}[\mathbf{x}],\{\mathbf{x}, 1,31\}]]$

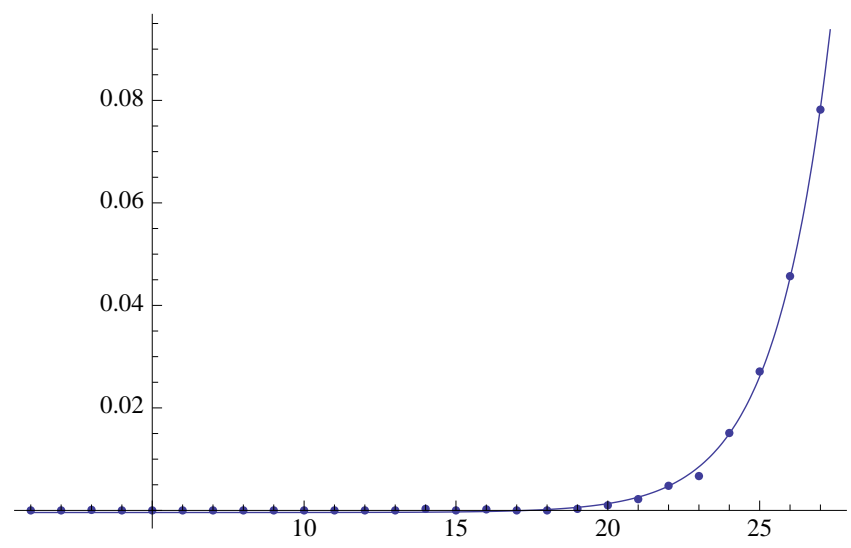

$\mathrm{X} 544 \mathrm{exp}=$

NonlinearModelFit $\left[\mathbf{X} 544\right.$ data $[[14 ; ; 28]], b+d 0 * a^{\wedge} \mathbf{x},\{b, d 0, a\}, \mathbf{x}$, MaxIterations $\left.\rightarrow 1000\right]$

FittedModel $\left[-0.000889306+1.44809 \times 10^{-7} \ll 19 \gg^{x}\right]$

X544exp [ BestFit"]

$-0.000889306+1.44809 \times 10^{-7} 1.61614^{x}$

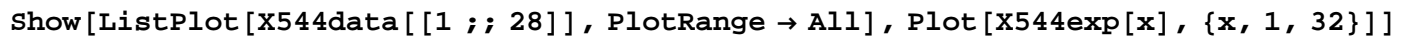

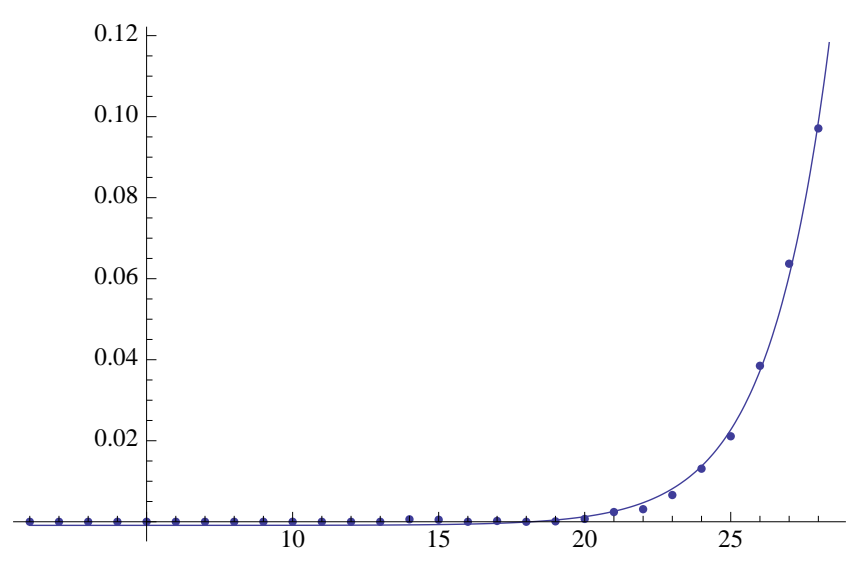

$\mathrm{X551exp}=$

NonlinearModelFit $\left[\mathbf{x} 551\right.$ data $[[13 ; ; 27]], b+d 0 * a^{\wedge} \mathbf{x},\{b, d 0, a\}, \mathbf{x}$, MaxIterations $\left.\rightarrow 1000\right]$

FittedModel $\left[-0.000287049+4.57546 \times 10^{-8} \ll 18 \gg^{x}\right]$

X551exp ["BestFit"]

$-0.000287049+4.57546 \times 10^{-8} 1.69713^{x}$ 


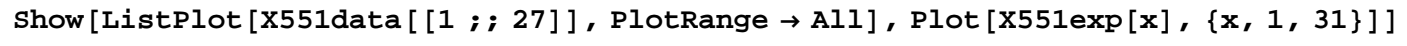

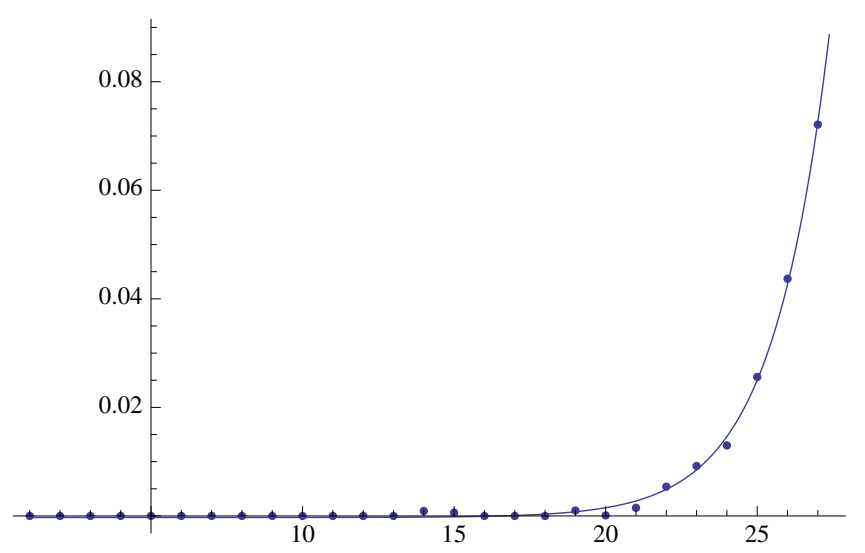

$\mathrm{x} 552 \mathrm{exp}=$

NonlinearModelFit [X552data $[13 ; ; 27]], b+d 0 * a^{\wedge} \mathbf{x},\{b, d 0, a\}, \mathbf{x}$, MaxIterations $\left.\rightarrow 1000\right]$

FittedModel $\left[-0.000357023+2.83702 \times 10^{-8} \ll 19 \gg^{x}\right]$

X552exp ["BestFit"]

$-0.000357023+2.83702 \times 10^{-8} 1.72954^{x}$

Show $[$ ListPlot $[X 552$ data $[1 ; ; 27]], P l o t R a n g e \rightarrow A l 1], P 1 o t[X 552 \exp [\mathbf{x}],\{x, 1,31\}]]$

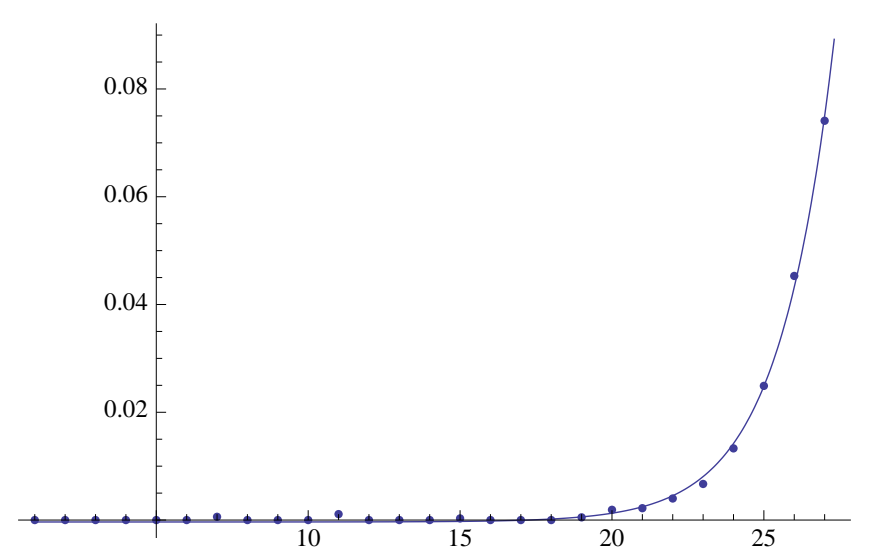

$\mathrm{x} 553 \mathrm{exp}=$

NonlinearModelFit [X553data $[13 ; ; 27]], b+d 0 * a^{\wedge} \mathbf{x},\{b, d 0, a\}, \mathbf{x}$, MaxIterations $\left.\rightarrow 1000\right]$

FittedModel $\left[-0.000262054+4.40371 \times 10^{-8} \ll 19 \gg^{x}\right]$

X553exp ["BestFit"]

$-0.000262054+4.40371 \times 10^{-8} 1.70584^{x}$ 
Show [ListPlot $[\mathrm{X553data}[[1 ; ; 27]], \mathrm{PlotRange} \rightarrow \mathrm{All}], \mathrm{Plot}[\mathrm{X553exp}[\mathbf{x}],\{\mathbf{x}, 1,31\}]]$

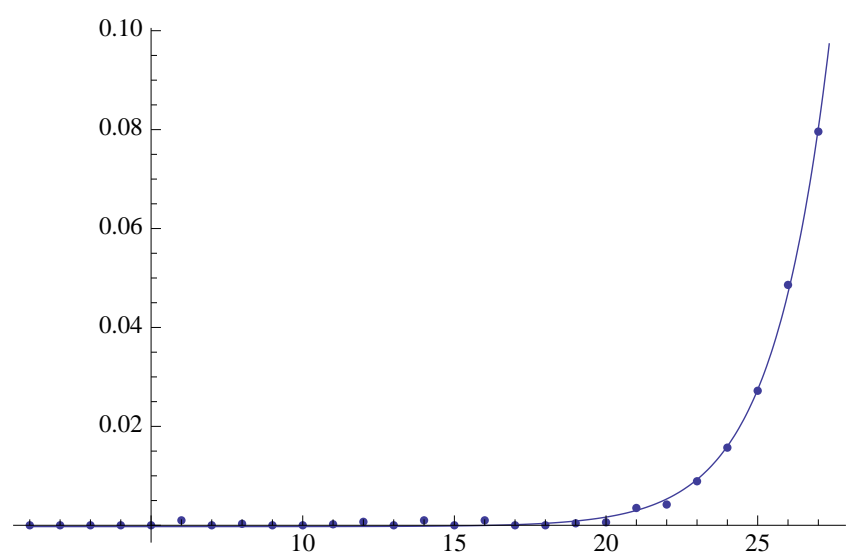

$\mathrm{X} 554 \mathrm{exp}=$

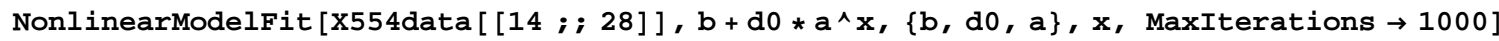

FittedModel $\left[-0.000684677+8.24391 \times 10^{-8} \ll 19 \gg \times\right.$

X554exp ["BestFit"]

$-0.000684677+8.24391 \times 10^{-8} 1.65668^{x}$

Show [ListPlot $[\mathrm{X} 554$ data $[1 ; ; 28]], \mathrm{PlotRange} \rightarrow \mathrm{All}], \mathrm{Plot}[\mathrm{X} 554 \exp [\mathbf{x}],\{\mathbf{x}, 1,32\}]]$

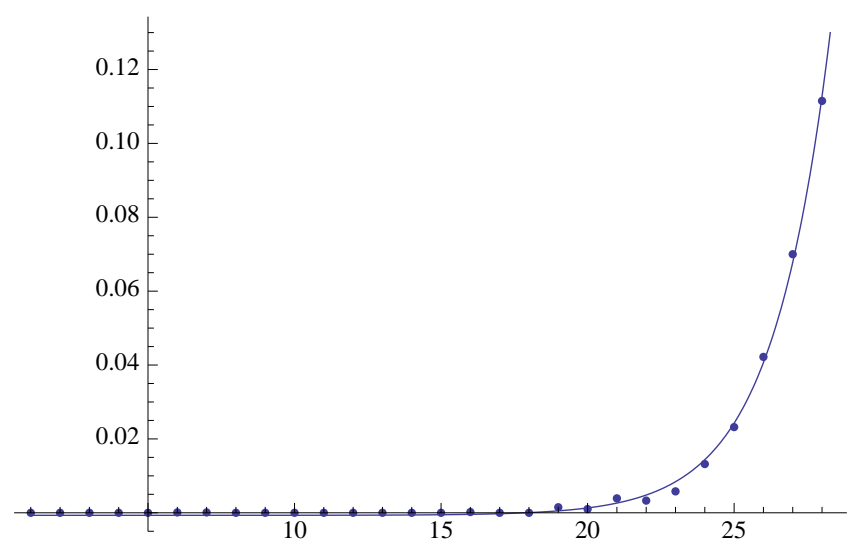

$\mathrm{x} 611 \mathrm{exp}=$

NonlinearModelFit $[\mathbf{x} 611$ data $[18 ; ; 30]], b+d 0 * a^{\wedge} \mathbf{x},\{b, d 0, a\}, \mathbf{x}$, MaxIterations $\left.\rightarrow 1000\right]$

FittedModel $\left[-0.0000908231+2.98514 \times 10^{-9} \ll 19 \gg^{x}\right]$

x611exp ["BestFit"]

$-0.0000908231+2.98514 \times 10^{-9} 1.74162^{x}$ 
Show [ListPlot $[\mathrm{X} 611$ data $[[1 ; ; 30]], \mathrm{PlotRange} \rightarrow \mathrm{All}], \mathrm{Plot}[\mathrm{X} 611 \exp [\mathbf{x}],\{\mathbf{x}, 1,35\}]]$

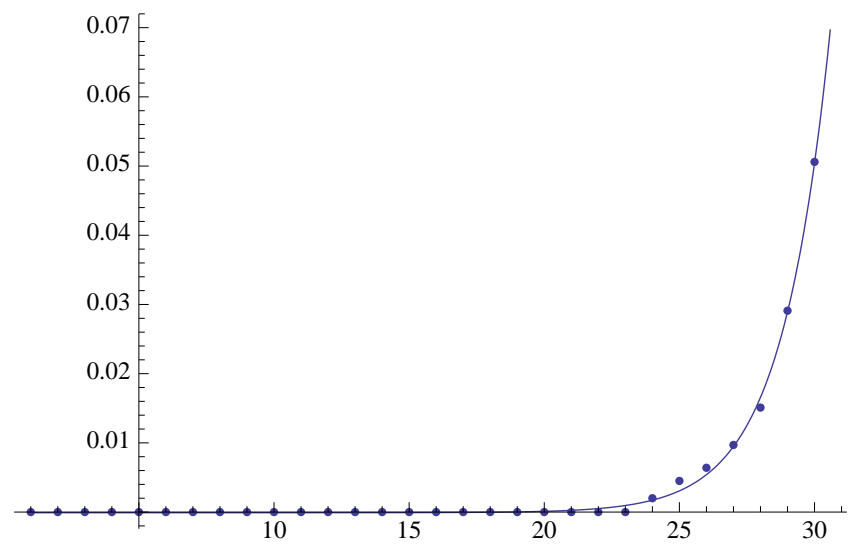

$\mathrm{x} 612 \mathrm{exp}=$

NonlinearModelFit [X612data $[18 ; ; 30]], b+d 0 * a^{\wedge} \mathbf{x},\{b, d 0, a\}, \mathbf{x}$, MaxIterations $\left.\rightarrow 1000\right]$

FittedModel $\left[-0.000518142+4.44221 \times 10^{-9} \ll 19>^{x}\right]$

x612exp ["BestFit"]

$-0.000518142+4.44221 \times 10^{-9} 1.72556^{x}$

Show $[$ ListPlot $[X 612$ data $[1 ; ; 30]], P l o t R a n g e \rightarrow A l l], P 1 o t[X 612 \exp [\mathbf{x}],\{\mathbf{x}, 1,35\}]]$

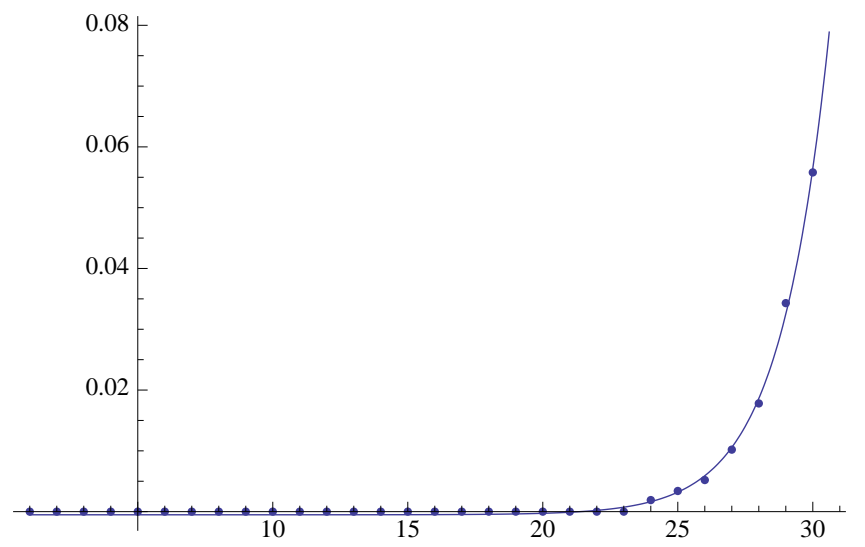

$\mathrm{x} 613 \mathrm{exp}=$

NonlinearModelFit [X613data $[18 ; ; 30]], b+d 0 * a^{\wedge} \mathbf{x},\{b, d 0, a\}, \mathbf{x}$, MaxIterations $\left.\rightarrow 1000\right]$

FittedModel $\left[-0.000404431+1.63179 \times 10^{-9} \ll 19 \gg^{x}\right]$

x613exp ["BestFit"]

$-0.000404431+1.63179 \times 10^{-9} 1.78923^{x}$ 
Show [ListPlot $[\mathrm{X} 613 \operatorname{dat}[[1 ; ; 30]], \mathrm{PlotRange} \rightarrow \mathrm{All}], \mathrm{Plot}[\mathrm{X613exp}[\mathbf{x}],\{\mathbf{x}, 1,35\}]]$

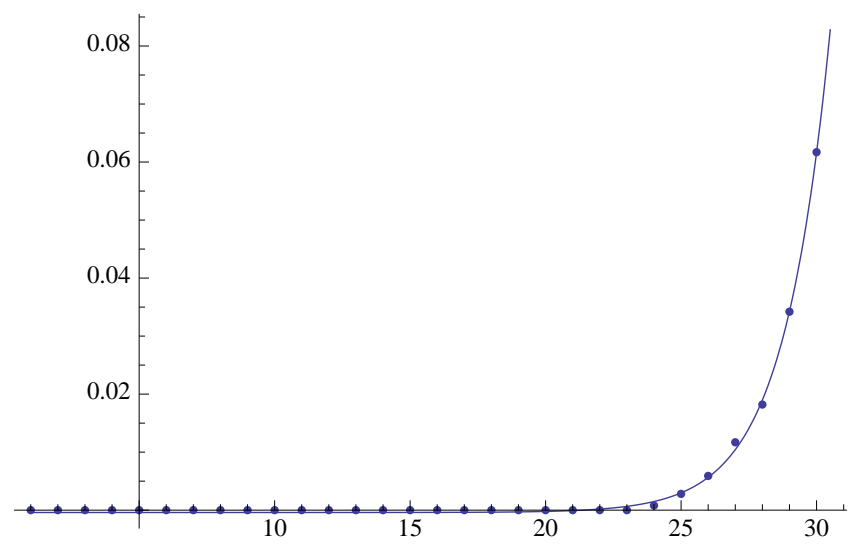

$\mathrm{x} 614 \mathrm{exp}=$

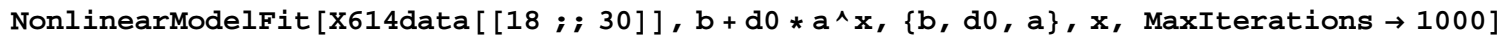

FittedModel $\left[-0.000431056+2.66141 \times 10^{-9} \ll 18 \gg \times\right.$

x614exp ["BestFit"]

$-0.000431056+2.66141 \times 10^{-9} 1.76091^{x}$

Show [ListPlot $[\mathrm{X} 614$ data $[1 ; ; 30]], \mathrm{PlotRange} \rightarrow \mathrm{All}], \mathrm{Plot}[\mathrm{X} 614 \exp [\mathbf{x}],\{\mathbf{x}, 1,35\}]]$

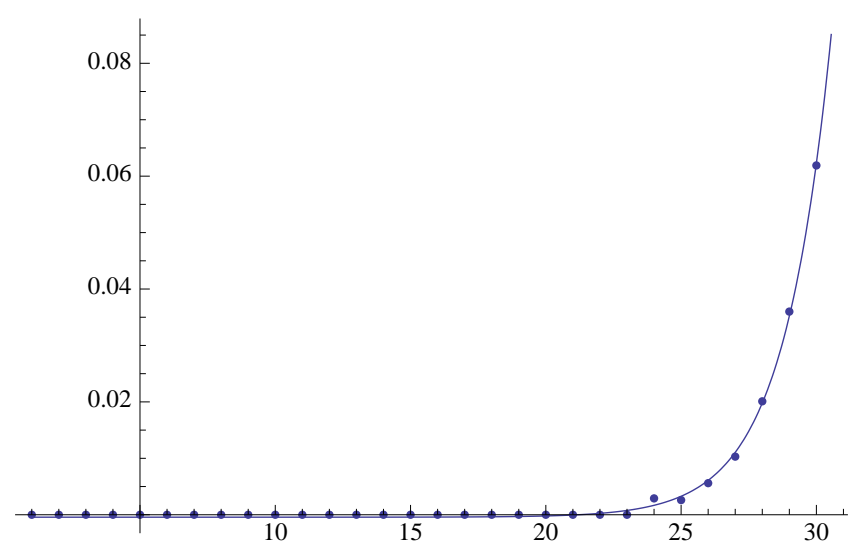

$\mathrm{x} 621 \mathrm{exp}=$

NonlinearModelFit $[\mathrm{X} 621$ data $[[15 ; ; 31]], b+d 0 * a \wedge \mathbf{x},\{b, d 0, a\}, \mathbf{x}$, MaxIterations $\rightarrow 1000]$

FittedModel $\left[-0.000225625+8.27961 \times 10^{-9} \ll 19 \gg^{x}\right]$

x621exp ["BestFit"]

$-0.000225625+8.27961 \times 10^{-9} 1.68574^{x}$ 
Show $[$ ListPlot $[\mathrm{X} 621$ data $[1 ; ; 31]], \mathrm{PlotRange} \rightarrow \mathrm{All}], \mathrm{P} \operatorname{lot}[\mathrm{X} 621 \exp [\mathbf{x}],\{\mathbf{x}, 1,35\}]]$

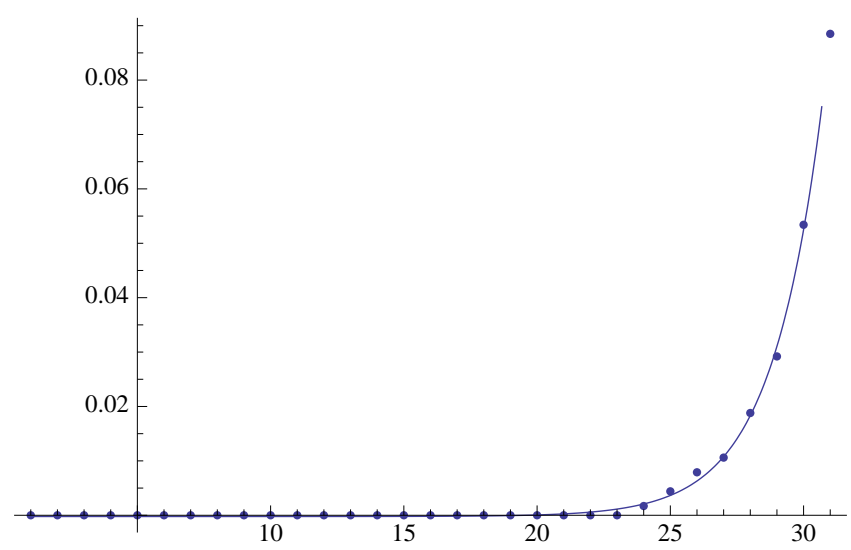

$\mathrm{x} 622 \mathrm{exp}=$

NonlinearModelFit [X622data $[15 ; ; 31]], b+d 0 * a^{\wedge} \mathbf{x},\{b, d 0, a\}, \mathbf{x}$, MaxIterations $\left.\rightarrow 1000\right]$

FittedModel $\left[-0.000538851+8.27626 \times 10^{-9} \ll 19 \gg^{x}\right]$

x622exp ["BestFit"]

$-0.000538851+8.27626 \times 10^{-9} 1.68927^{x}$

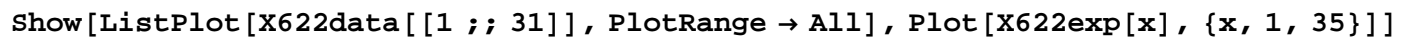

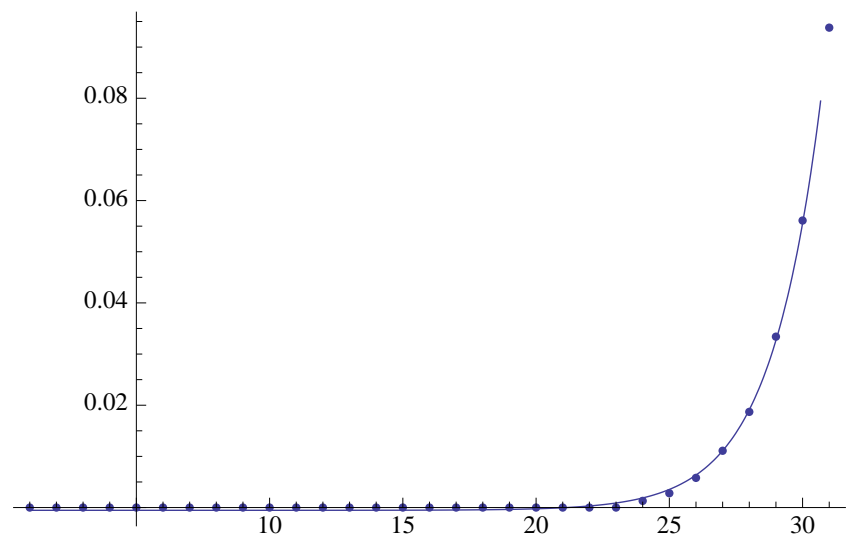

$\mathrm{x} 623 \exp =$

NonlinearModelFit $\left[\mathbf{X 6 2 3 d a t a}[[15 ; ; 31]], b+d 0 * a^{\wedge} \mathbf{x},\{b, d 0, a\}, \mathbf{x}\right.$, MaxIterations $\left.\rightarrow 1000\right]$

FittedModel $\left[-0.000440008+8.04808 \times 10^{-9} \ll 19 \gg^{x}\right]$

$\mathrm{x} 623 \exp [$ "BestFit"]

$-0.000440008+8.04808 \times 10^{-9} 1.69071^{x}$ 
Show $[$ ListPlot $[\mathrm{X} 623$ data $[1 ; ; 31]], \mathrm{PlotRange} \rightarrow \mathrm{All}], \mathrm{P} \operatorname{lot}[\mathrm{X} 623 \exp [\mathbf{x}],\{\mathbf{x}, 1,35\}]]$

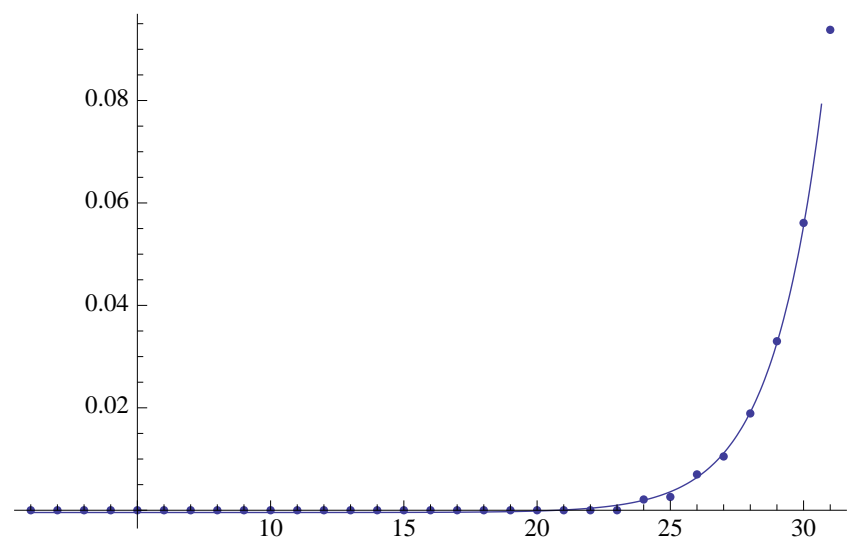

$\mathrm{x} 624 \mathrm{exp}=$

NonlinearModelFit [X624data $[[15 ; ; 31]], b+d 0 * a^{\wedge} \mathbf{x},\{b, d 0, a\}, \mathbf{x}$, MaxIterations $\left.\rightarrow 1000\right]$

FittedModel $\left[-0.00061082+1.01173 \times 10^{-8} \ll 19 \gg^{x}\right]$

x624exp [ BestFit"]

$-0.00061082+1.01173 \times 10^{-8} 1.67564^{x}$

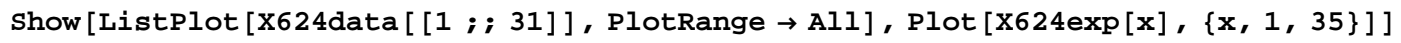

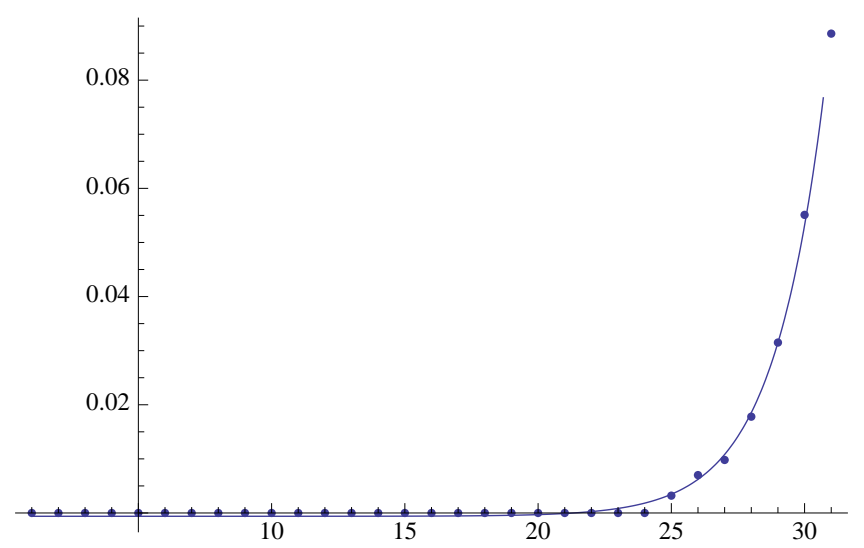

$\mathrm{x} 631 \mathrm{exp}=$

NonlinearModelFit $[\mathrm{X} 631$ data $[15 ; ; 31]], b+d 0 * a \wedge \mathbf{x},\{b, d 0, a\}, \mathbf{x}, \operatorname{MaxIterations} \rightarrow 1000]$

FittedModel $\left[-0.000361452+8.02935 \times 10^{-9} \ll 18 \gg^{x}\right]$

x631exp ["BestFit"]

$-0.000361452+8.02935 \times 10^{-9} 1.69356^{x}$ 


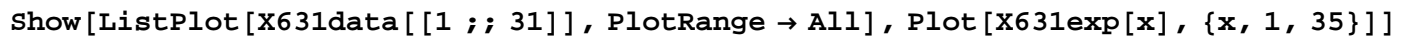

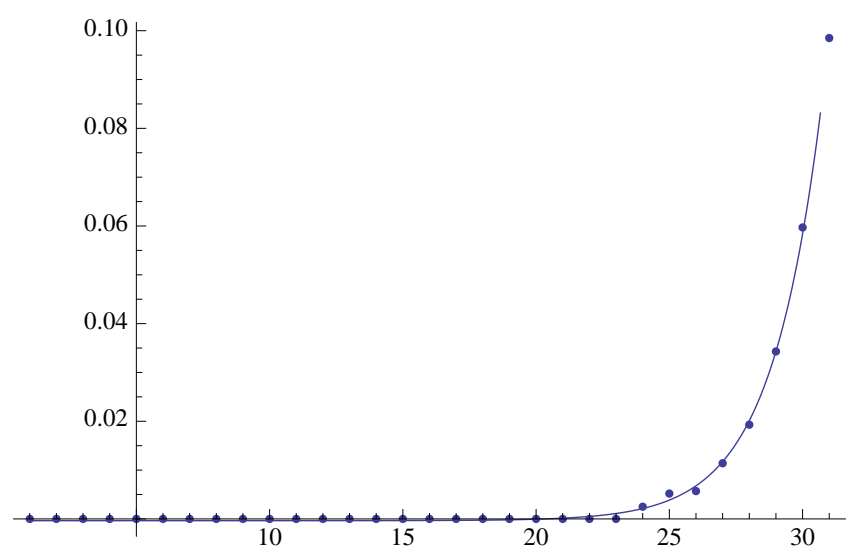

$\mathrm{x} 632 \mathrm{exp}=$

NonlinearModelFit $\left[\mathbf{x 6 3 2 d a t a}[[15 ; ; 31]], b+d 0 * a^{\wedge} \mathbf{x},\{b, d 0, a\}, \mathbf{x}\right.$, MaxIterations $\left.\rightarrow 1000\right]$

FittedModel $\left[-0.000649099+7.51224 \times 10^{-9} \ll 19>^{x}\right]$

x632exp ["BestFit"]

$-0.000649099+7.51224 \times 10^{-9} 1.6991^{x}$

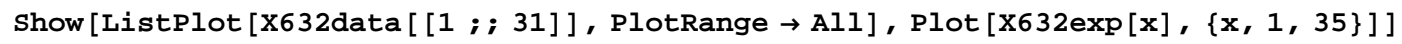

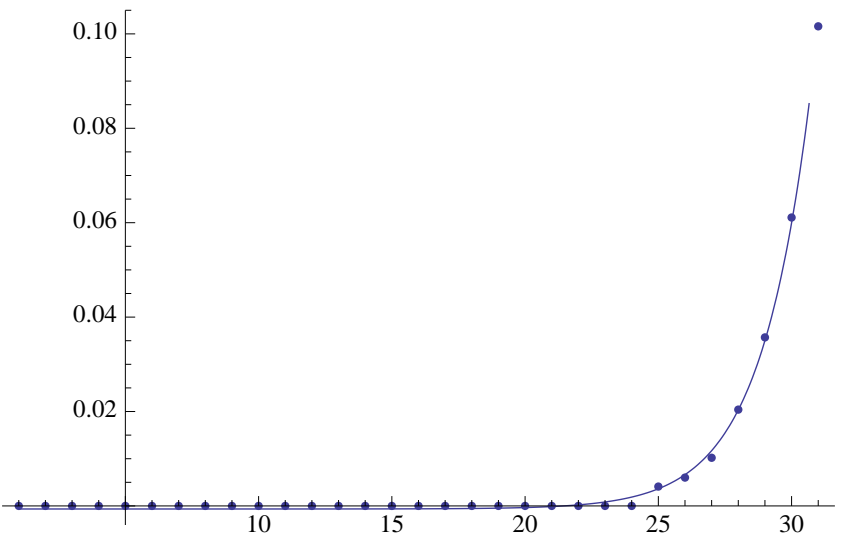

x633exp $=$

NonlinearModelFit [X633data $[15 ; ; 31]], b+d 0 * a^{\wedge} \mathbf{x},\{b, d 0, a\}, \mathbf{x}$, MaxIterations $\left.\rightarrow 1000\right]$

FittedModel $\left[-0.00073083+1.83628 \times 10^{-8} \ll 19 \gg^{x}\right]$

X633exp ["BestFit"]

$-0.00073083+1.83628 \times 10^{-8} 1.6502^{x}$ 
Show [ListPlot $[\mathrm{X633data}[[1 ; ; 31]], \mathrm{PlotRange} \rightarrow \mathrm{All}], \mathrm{Plot}[\mathrm{X633exp}[\mathbf{x}],\{\mathbf{x}, 1,35\}]]$

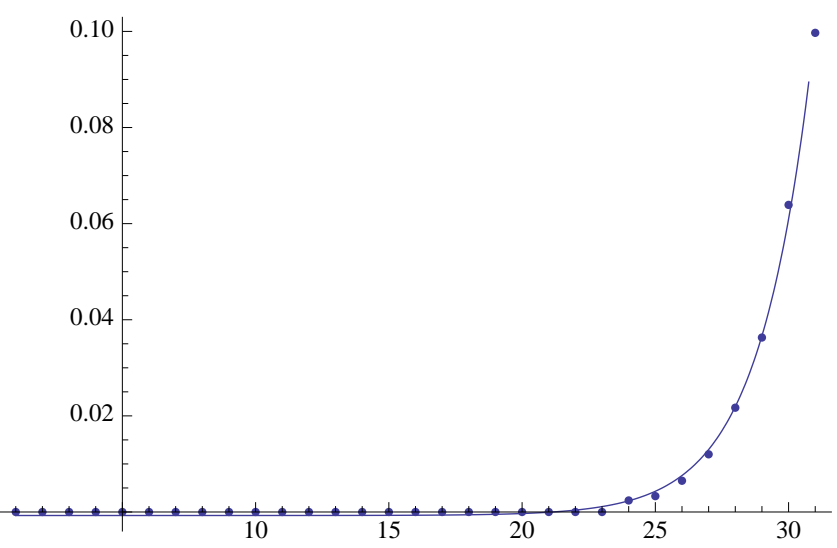

$\mathrm{x} 634 \mathrm{exp}=$

NonlinearModelFit $[\mathbf{x} 634$ data $[15 ; ; 31]], b+d 0 * a \wedge \mathbf{x},\{b, d 0, a\}, \mathbf{x}$, MaxIterations $\rightarrow 1000]$

FittedModel $\left[-0.000689452+9.46028 \times 10^{-9} \ll 17 \gg^{x}\right]$

x634exp ["BestFit"]

$-0.000689452+9.46028 \times 10^{-9} 1.68271^{x}$

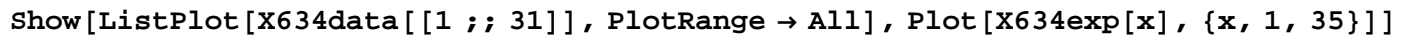

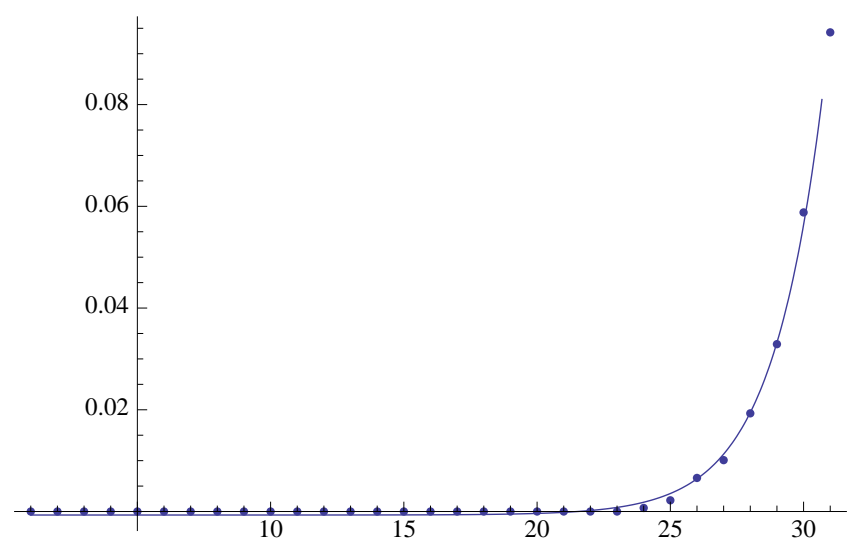

$\mathrm{x} 641 \mathrm{exp}=$

NonlinearModelFit $[\mathbf{X} 641$ data $[15 ; ; 31]], b+d 0 * a \wedge \mathbf{x},\{b, d 0, a\}, \mathbf{x}, \operatorname{MaxIterations} \rightarrow 1000]$

FittedModel $\left[-0.000648255+7.73739 \times 10^{-9} \ll 19 \gg^{x}\right]$

x641exp ["BestFit"]

$-0.000648255+7.73739 \times 10^{-9} 1.68535^{x}$ 


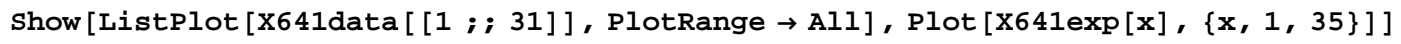

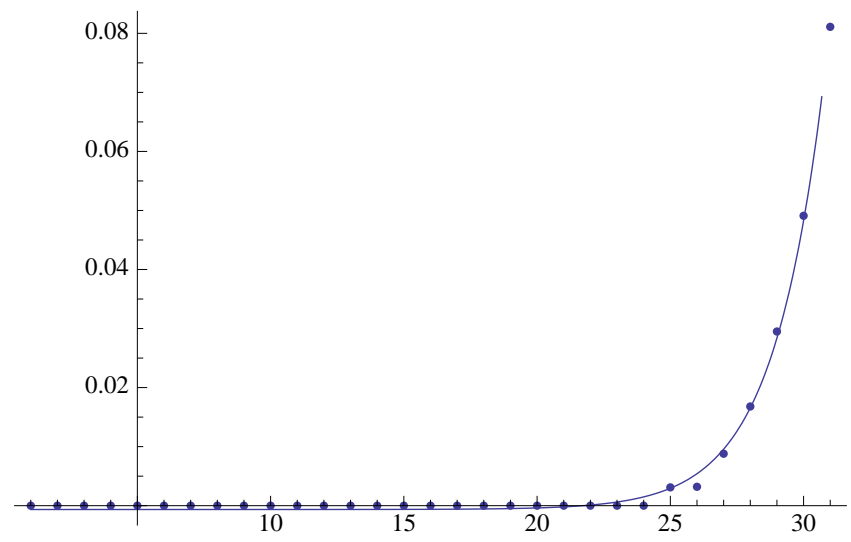

$\mathrm{x} 642 \mathrm{exp}=$

NonlinearModelFit $[\mathbf{X} 642$ data $[15 ; ; 31]], b+d 0 * a \wedge \mathbf{x},\{b, d 0, a\}, \mathbf{x}$, MaxIterations $\rightarrow 1000]$

FittedModel $\left[-0.000661835+1.13516 \times 10^{-8} \ll 19 \gg^{x}\right]$

$\mathrm{x} 642 \exp [$ "BestFit"]

$-0.000661835+1.13516 \times 10^{-8} 1.65859^{x}$

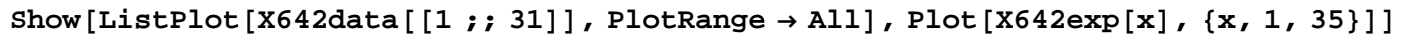

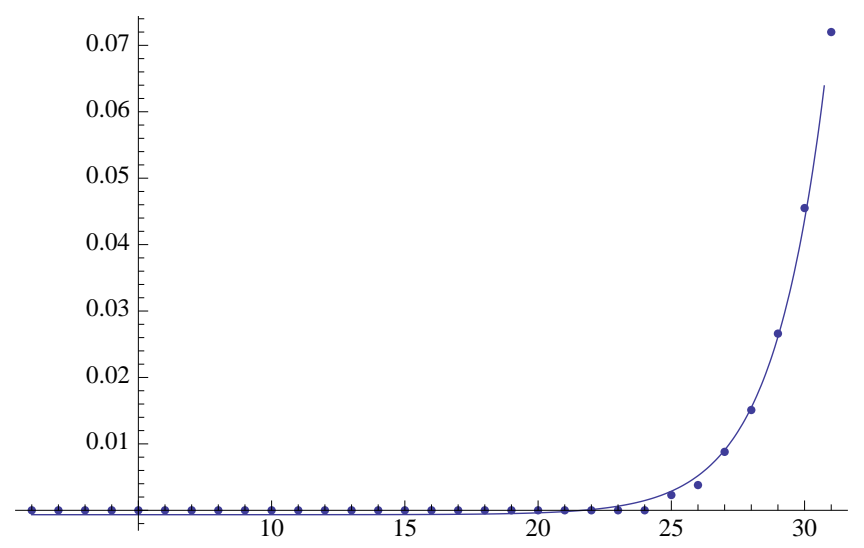

$\mathrm{x} 643 \mathrm{exp}=$

NonlinearModelFit $\left[\mathbf{x} 643\right.$ data $[[15 ; ; 32]], b+d 0 * a^{\wedge} \mathbf{x},\{b, d 0, a\}, \mathbf{x}$, MaxIterations $\left.\rightarrow 1000\right]$

FittedModel $\left[-0.000810245+3.94578 \times 10^{-8} \ll 19 \gg^{x}\right]$

x643exp ["BestFit"]

$-0.000810245+3.94578 \times 10^{-8} 1.58553^{x}$ 
Show [ListPlot $[\mathrm{X} 643 \operatorname{dat}[[1 ; ; 32]], \mathrm{PlotRange} \rightarrow \mathrm{All}], \mathrm{Plot}[\mathrm{X643exp}[\mathbf{x}],\{\mathbf{x}, 1,37\}]]$

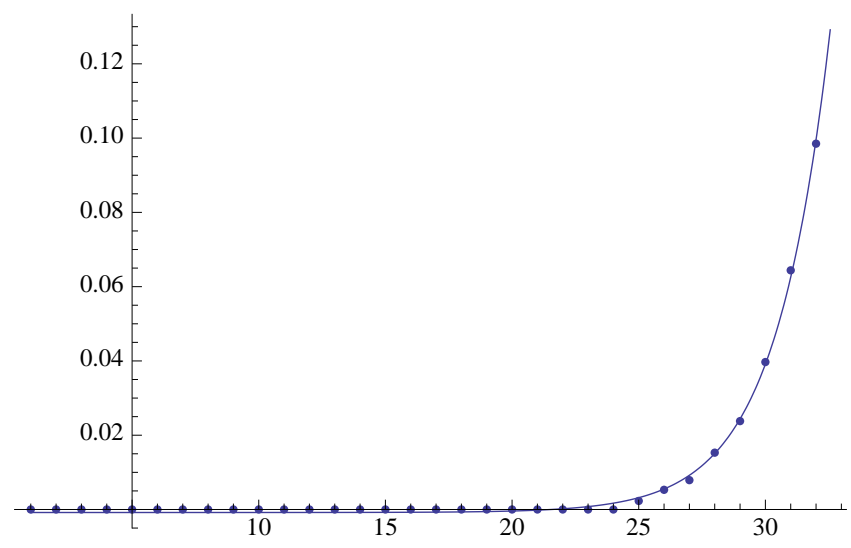

$\mathrm{x} 644 \mathrm{exp}=$

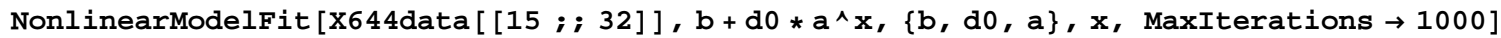

FittedModel $\left[-0.00102383+2.40278 \times 10^{-8} \ll 18 \gg^{x}\right]$

x644exp ["BestFit"]

$-0.00102383+2.40278 \times 10^{-8} 1.61025^{x}$

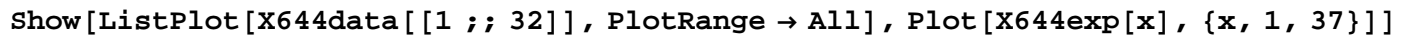

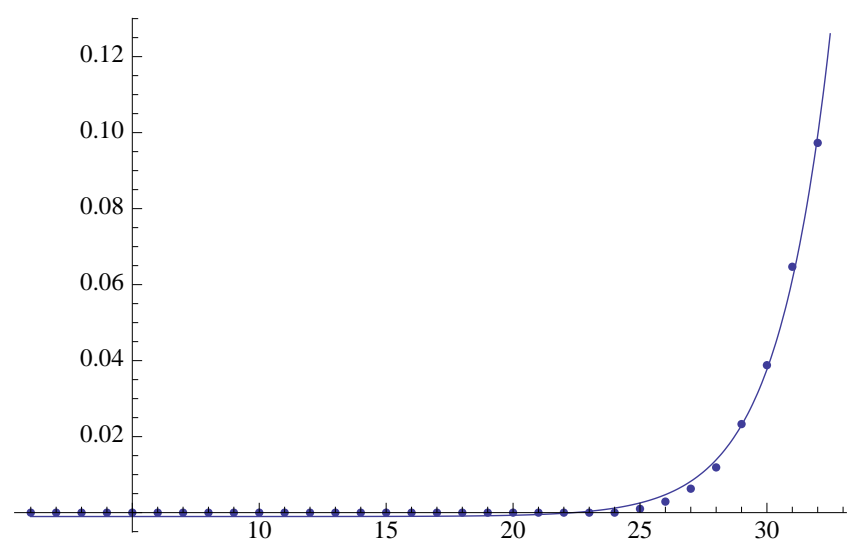

$\mathrm{x} 651 \mathrm{exp}=$

NonlinearModelFit $\left[\mathbf{X 6 5 1 d a t a}[[15 ; ; 31]], b+d 0 * a^{\wedge} \mathbf{x},\{b, d 0, a\}, \mathbf{x}\right.$, MaxIterations $\left.\rightarrow 1000\right]$

FittedModel $\left[-0.000700728+1.03121 \times 10^{-8} \ll 19 \gg^{x}\right]$

x651exp ["BestFit"]

$-0.000700728+1.03121 \times 10^{-8} 1.67175^{x}$ 
Show $[$ ListPlot $[\mathrm{X651data}[[1 ; ; 31]], \mathrm{PlotRange} \rightarrow \mathrm{All}], \mathrm{Plot}[\mathrm{X651} \exp [\mathbf{x}],\{\mathbf{x}, 1,37\}]$

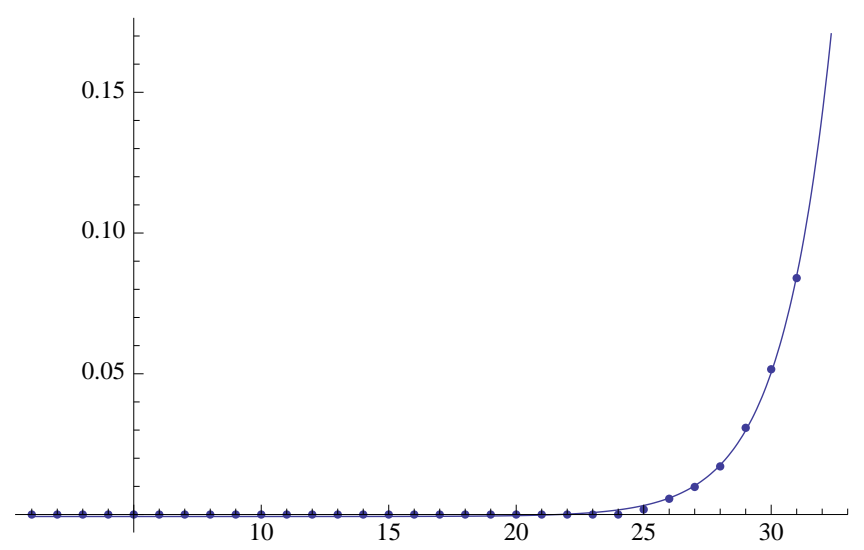

$\mathrm{x} 652 \mathrm{exp}=$

NonlinearModelFit [x652data $[15 ; ; 31]], b+d 0 * a^{\wedge} \mathbf{x},\{b, d 0, a\}, \mathbf{x}$, MaxIterations $\left.\rightarrow 1000\right]$

FittedModel $\left[-0.00067847+8.8 \times 10^{-9} \ll 19 \gg^{x}\right]$

x652exp ["BestFit"]

$-0.00067847+8.8 \times 10^{-9} 1.68613^{x}$

Show [ListPlot $[\mathrm{X652data}[[1 ; ; 31]], \mathrm{PlotRange} \rightarrow \mathrm{All}], \mathrm{Plot}[\mathrm{X652 \operatorname {exp }}[\mathbf{x}],\{\mathbf{x}, 1,36\}]]$

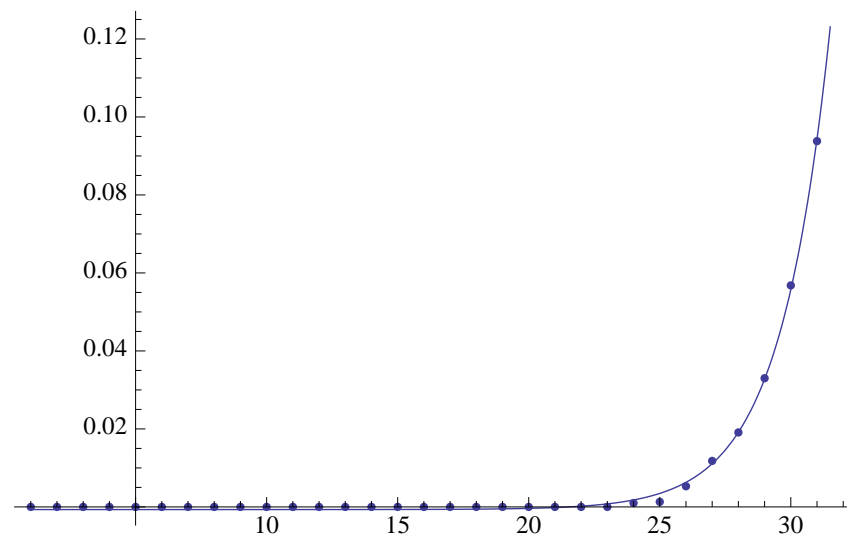

$\mathrm{x} 653 \mathrm{exp}=$

NonlinearModelFit [X653data $[15 ; ; 31]], b+d 0 * a \wedge x,\{b, d 0, a\}, \mathbf{x}$, MaxIterations $\rightarrow 1000]$

FittedModel $\left[-0.000706141+9.94051 \times 10^{-9} \ll 19 \gg^{x}\right]$

x653exp ["BestFit"]

$-0.000706141+9.94051 \times 10^{-9} 1.67423^{x}$ 
Show [ListPlot $[\mathrm{X653data}[[1 ; ; 31]], \mathrm{PlotRange} \rightarrow \mathrm{All}], \mathrm{Plot}[\mathrm{X653exp}[\mathbf{x}],\{\mathbf{x}, 1,36\}]]$

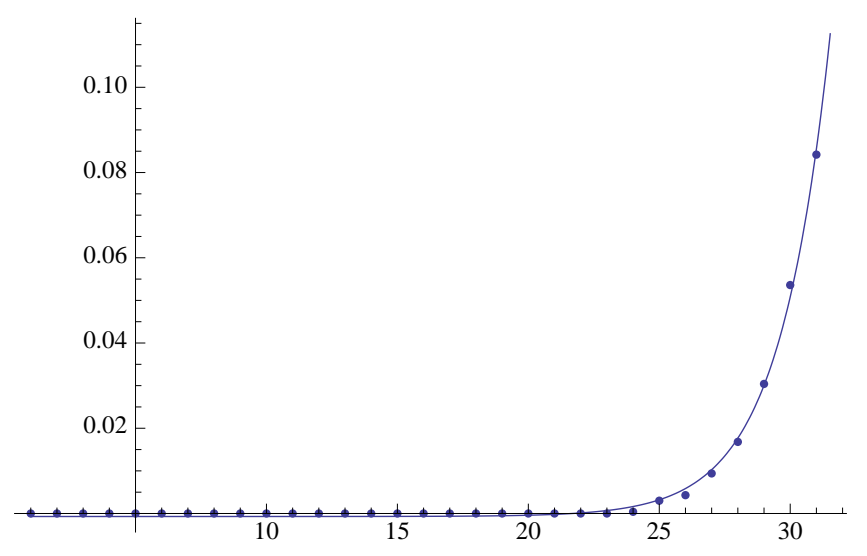

$\mathrm{x} 654 \mathrm{exp}=$

NonlinearModelFit $\left[\mathbf{X 6 5 4 d a t a}[[15 ; ; 31]], b+d 0 * a^{\wedge} \mathbf{x},\{b, d 0, a\}, \mathbf{x}\right.$, MaxIterations $\left.\rightarrow 1000\right]$

FittedModel $\left[-0.000464563+6.94442 \times 10^{-9} \ll 19 \gg^{x}\right]$

x654exp ["BestFit"]

$-0.000464563+6.94442 \times 10^{-9} 1.6828^{x}$ 

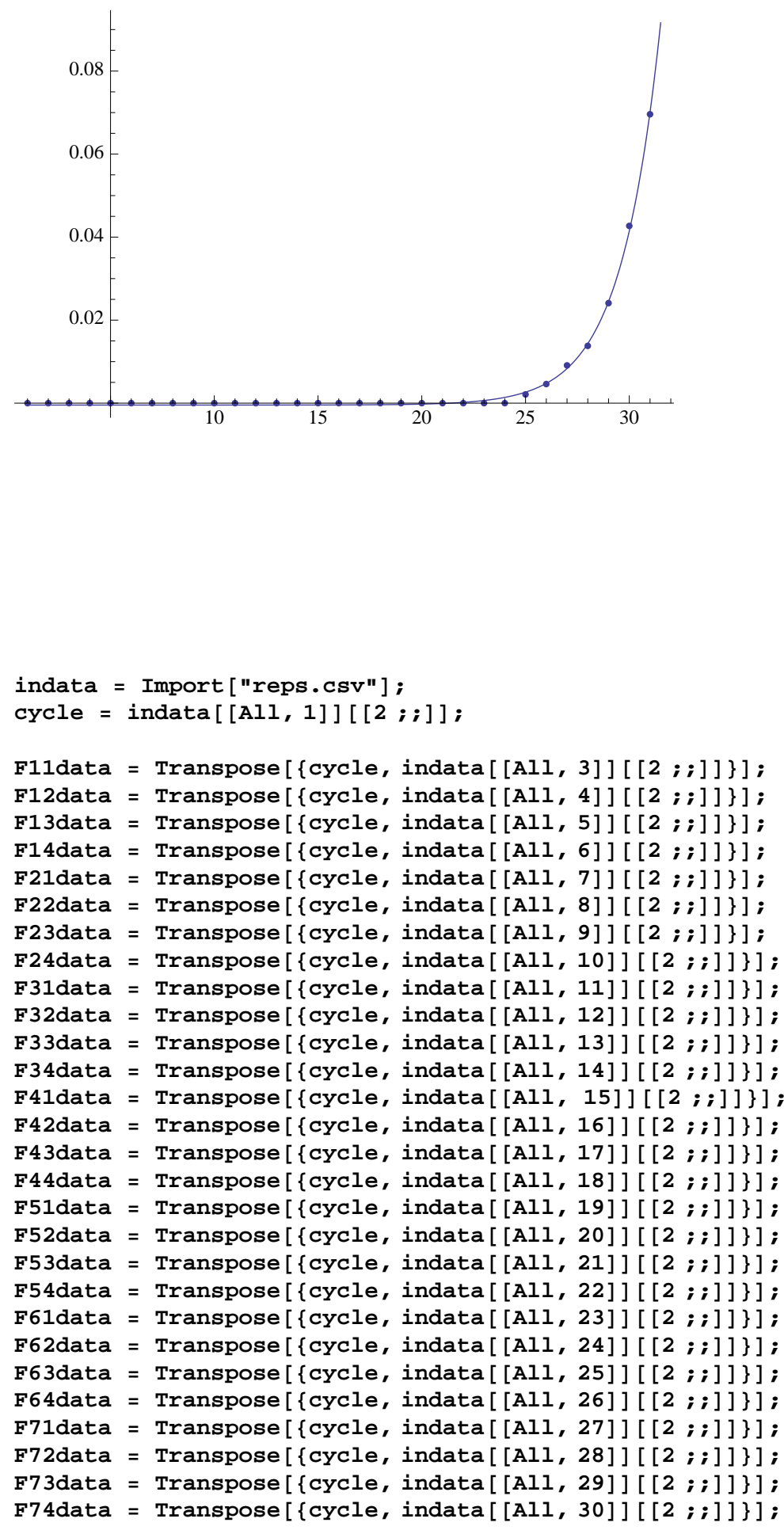
F11exp ["BestFit"]

$0.00100241+0.000365821 .74643^{x}$

Show [ListPlot $[F 11$ data $[1 ; ; 15]], \mathrm{PlotRange} \rightarrow \mathrm{All}], \mathrm{Plot}[\mathrm{F} 11 \exp [\mathbf{x}],\{\mathbf{x}, 1,17\}]]$

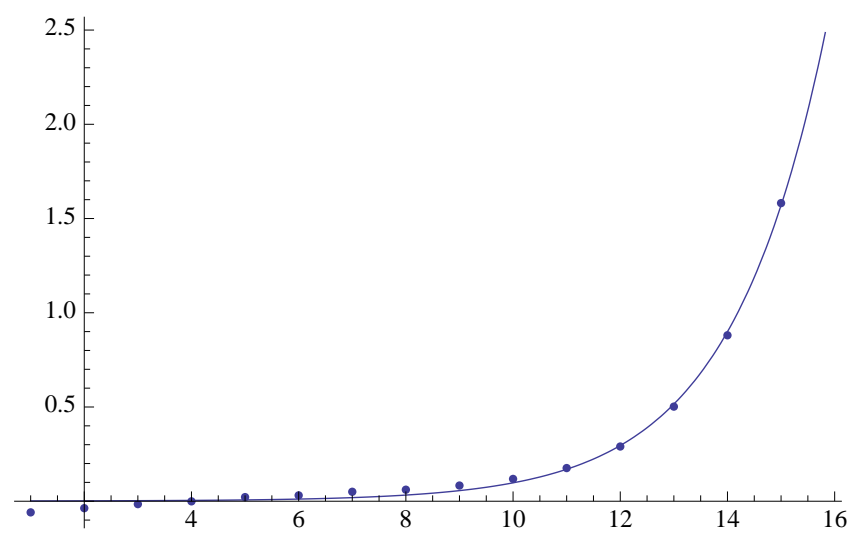

F12exp $=$ NonlinearModelFit $[F 12$ data $[1 ; ; 15]], b+d 0 * a^{\wedge} \mathbf{x},\{b$, d0, $a\}, \mathbf{x}$, MaxIterations $\left.\rightarrow 1000\right]$

FittedModel $\left[0.00134432+0.000404047 \ll 19 \gg^{x}\right.$

F12exp [ BestFit"]

$0.00134432+0.0004040471 .75124^{x}$

Show [ListPlot $[F 12$ data $[[1 ; ; 15]], \mathrm{PlotRange} \rightarrow \mathrm{All}], \mathrm{Plot}[\mathrm{F} 12 \exp [\mathbf{x}],\{\mathbf{x}, 1,17\}]]$

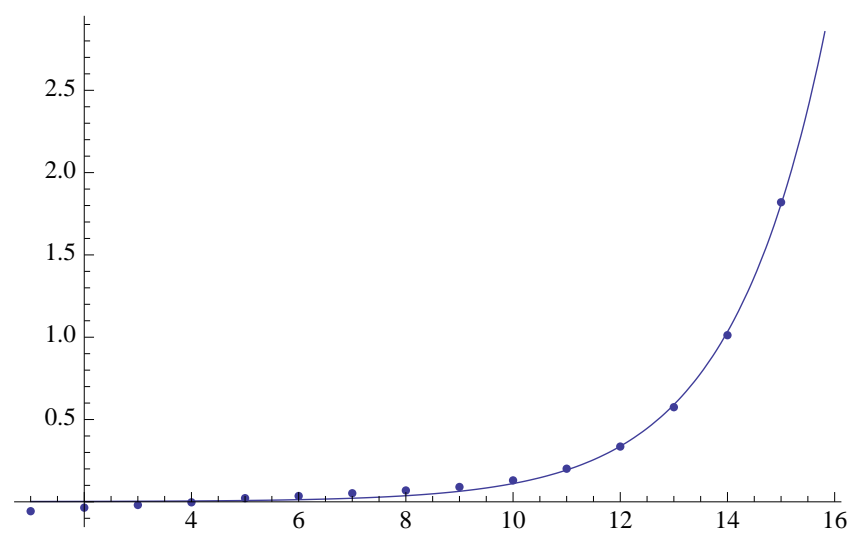

F13exp $=$ NonlinearModelFit $[F 13 d a t a[1 ; ; 15]], b+d 0 * a^{\wedge} \mathbf{x},\{b, d 0, a\}, \mathbf{x}$, MaxIterations $\left.\rightarrow 1000\right]$

FittedModel $\left[0.023234+0.00101156 \ll 19 \gg^{x}\right]$

F13exp [ BestFit"]

$0.023234+0.001011561 .63668^{x}$ 


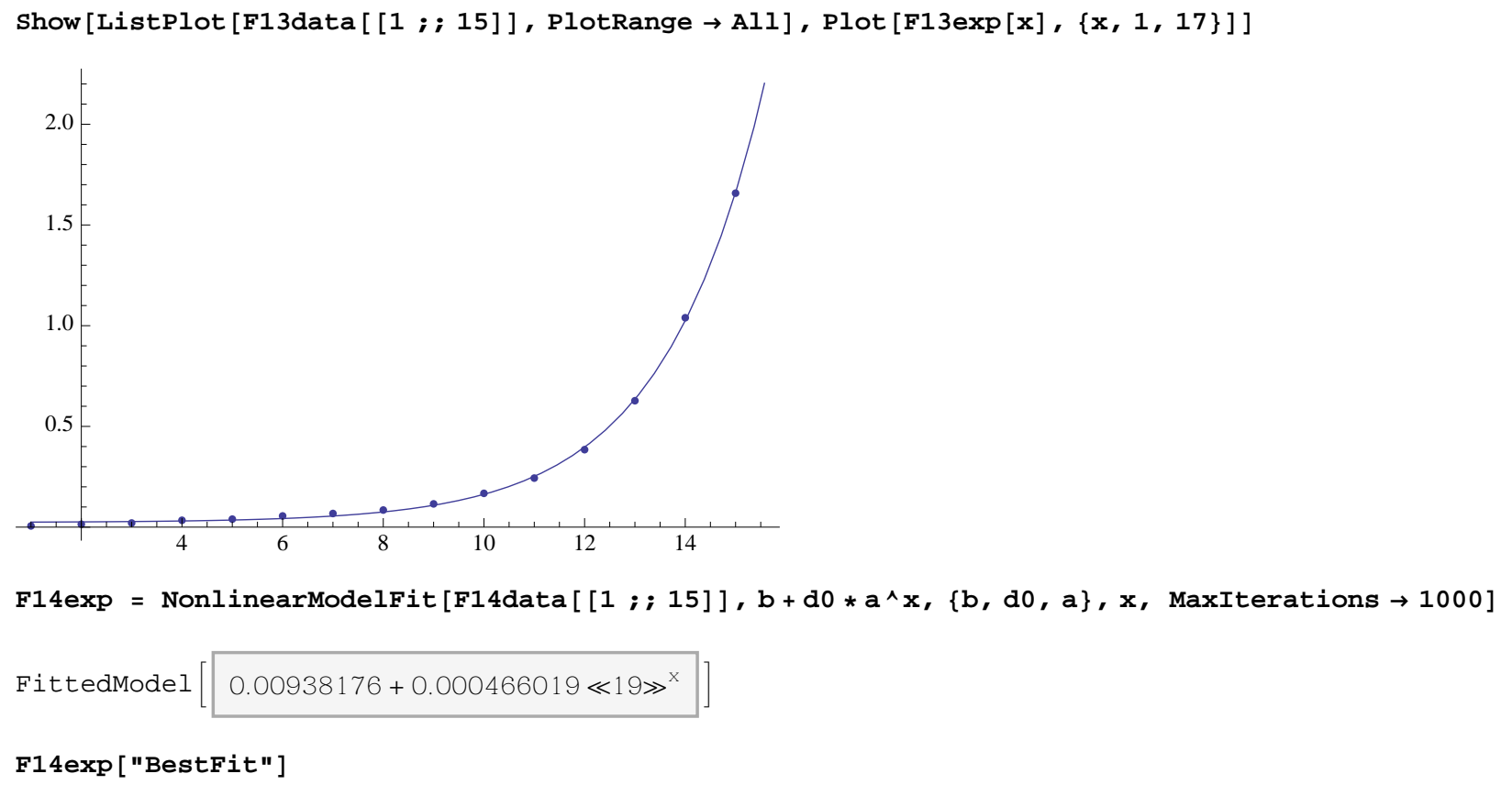

$0.00938176+0.0004660191 .69869^{x}$

Show [ListPlot $[F 14$ data $[1 ; ; 15]]$, PlotRange $\rightarrow A l 1], P l o t[F 14 \exp [\mathbf{x}],\{x, 1,17\}]]$

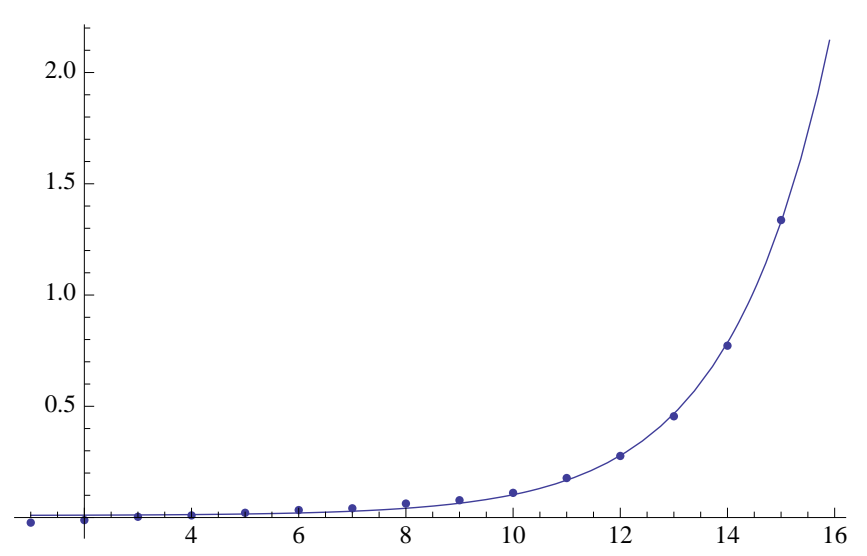

F21exp = NonlinearModelFit $[F 21$ data $[1 ; ; 18]], b+d 0 * a^{\wedge} \mathbf{x},\{b$, do, $\left.a\}, \mathbf{x}, \operatorname{MaxIterations} \rightarrow 1000\right]$

FittedModel $\left[0.0116202+0.0000396458 \ll 15 \gg^{x}\right]$

F21exp [ BestFit"]

$0.0116202+0.00003964581 .77338^{x}$ 
Show [ListPlot [F21data $[[1 ; ; 18]], \mathrm{PlotRange} \rightarrow \mathrm{All}], \mathrm{P} \operatorname{lot}[\mathrm{F} 21 \exp [\mathbf{x}],\{\mathbf{x}, 1,20\}]]$

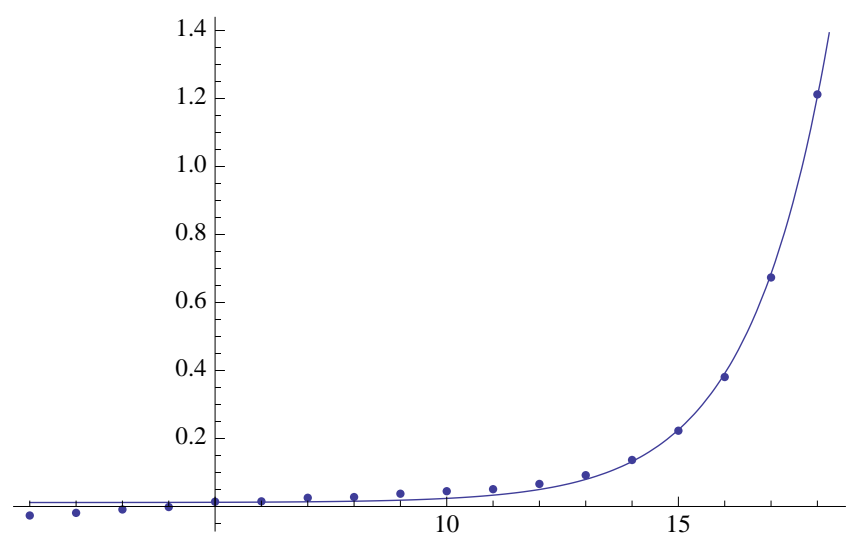

F22exp $=$ NonlinearModelFit [F22data $[1 ; ; 18]], b+d 0 * a^{\wedge} \mathbf{x},\{b, d 0, a\}, \mathbf{x}$, MaxIterations $\left.\rightarrow 1000\right]$

FittedModel $\left[0.0115172+0.0000431857 \ll 19 \gg^{x}\right.$

F22exp [ BestFit"]

$0.0115172+0.00004318571 .78057^{x}$

Show [ListPlot [F22data $[[1 ; ; 18]], \mathrm{PlotRange} \rightarrow \mathrm{All}], \mathrm{Plot}[\mathrm{F22exp}[\mathbf{x}],\{\mathbf{x}, 1,20\}]]$

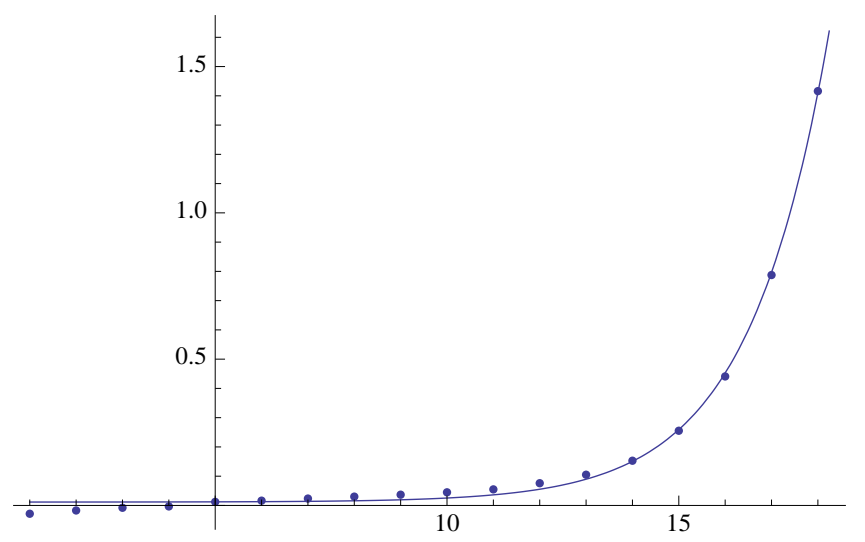

F23exp $=$ NonlinearModelFit $\left[F 23 d a t a[[1 ; ; 18]], b+d 0 * a^{\wedge} \mathbf{x},\{b\right.$, do, $\left.a\}, \mathbf{x}, \operatorname{MaxIterations~} \rightarrow 1000\right]$ FittedModel $\left[0.0251573+0.00020661 \ll 19 \gg^{x}\right.$

F23exp [ BestFit"]

$0.0251573+0.000206611 .64007^{x}$ 
Show [ListPlot [F23data $[1 ; ; 18]], \mathrm{PlotRange} \rightarrow \mathrm{All}], \mathrm{P} 1$ ot $[\mathrm{F2} 3 \exp [\mathbf{x}],\{\mathbf{x}, 1,20\}]]$

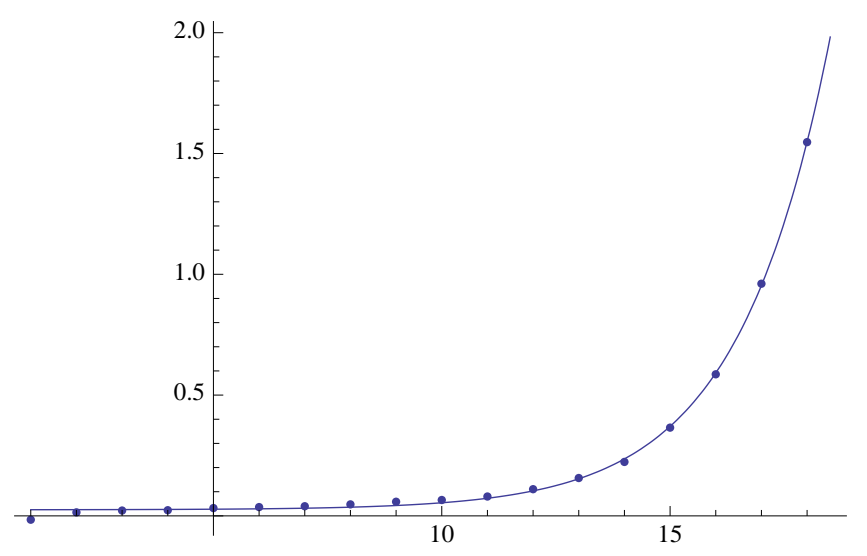

F24exp $=$ NonlinearModelFit $\left[\right.$ F24data $\left.[[1 ; ; 18]], b+d 0 * a^{\wedge} \mathbf{x},\{b, d 0, a\}, \mathbf{x}, \operatorname{MaxIterations} \rightarrow 1000\right]$

FittedModel $\left[0.0187197+0.000219314 \ll 18 \gg^{x}\right]$

F24exp [ BestFit"]

$0.0187197+0.0002193141 .67487^{x}$

Show [ListPlot [F24data $[1 ; ; 18]]$, PlotRange $\rightarrow$ All],$P 1 o t[F 24 \exp [\mathbf{x}],\{x, 1,20\}]]$

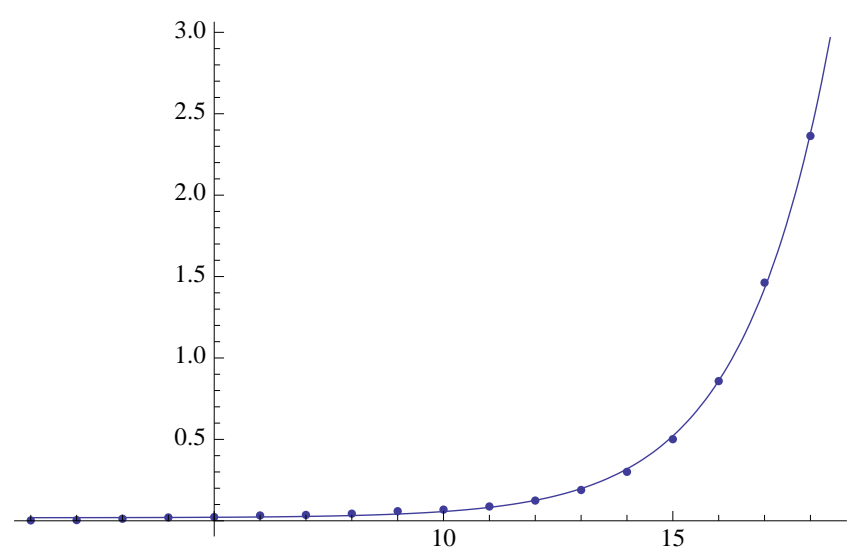

F31exp $=$ NonlinearModelFit $[F 31$ data $[1 ; ; 22]], b+d 0 * a^{\wedge} \mathbf{x},\{b, d 0, a\}, \mathbf{x}$, MaxIterations $\left.\rightarrow 1000\right]$

FittedModel $\left[0.0189188+4.87297 \times 10^{-6} \ll 19 \gg^{x}\right.$

F31exp [ BestFit"]

$0.0189188+4.87297 \times 10^{-6} 1.79821^{x}$ 
Show [ListPlot $[F 31$ data $[1 ; ; 22]]$, PlotRange $\rightarrow A l 1], P 1 o t[F 31 \exp [\mathbf{x}],\{\mathbf{x}, 1,25\}]]$

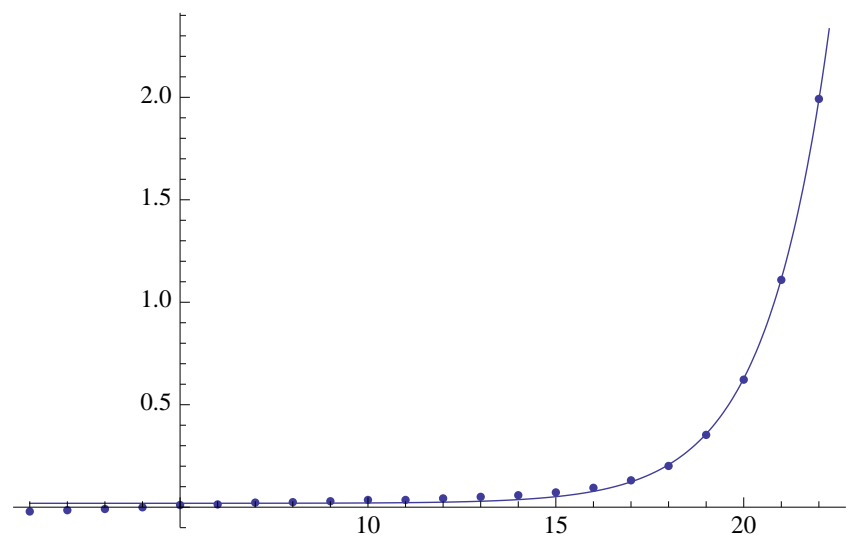

F32exp $=$ NonlinearModelFit $\left[\right.$ F32data $\left.[[1 ; ; 22]], b+d 0 * a^{\wedge} \mathbf{x},\{b, d 0, a\}, \mathbf{x}, \operatorname{MaxIterations} \rightarrow 1000\right]$

FittedModel $\left[0.0159663+5.17298 \times 10^{-6} \ll 19 \gg^{x}\right]$

F32exp [ BestFit"]

$0.0159663+5.17298 \times 10^{-6} 1.80446^{x}$

Show [ListPlot [F32data $[[1 ; ; 22]], P l o t R a n g e \rightarrow A l l], P l o t[F 32 \exp [\mathbf{x}],\{\mathbf{x}, 1,25\}]]$

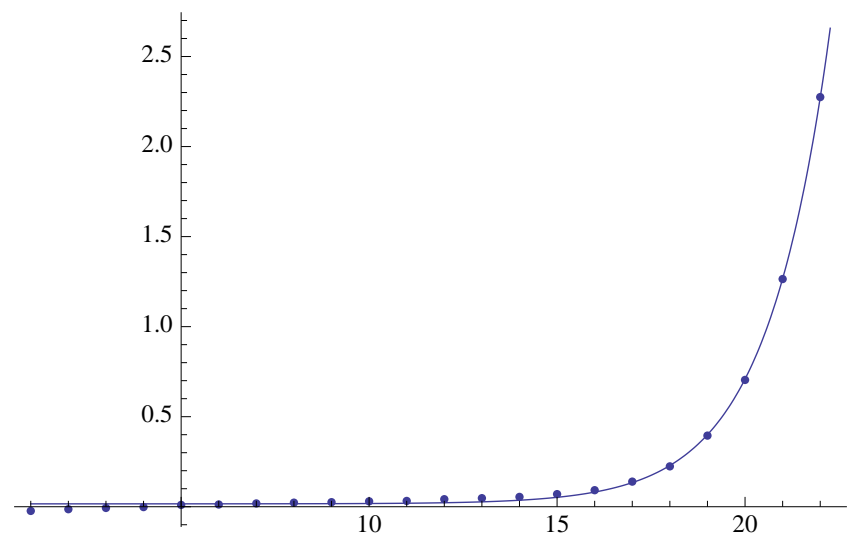

F33exp $=$ NonlinearModelFit $\left[F 33\right.$ data $\left.[[1 ; ; 22]], b+d 0 * a^{\wedge} \mathbf{x},\{b, d 0, a\}, \mathbf{x}, \operatorname{MaxIterations} \rightarrow 1000\right]$

FittedModel $\left[0.0310752+0.0000869497 \ll 19 \gg^{x}\right.$

F33exp [ BestFit"]

$0.0310752+0.00008694971 .59358^{x}$ 
Show [ListPlot [F33data $[1 ; ; 22]], \mathrm{PlotRange} \rightarrow \mathrm{All}], \mathrm{Plot}[\mathrm{F} 33 \exp [\mathbf{x}],\{\mathbf{x}, 1,25\}]]$

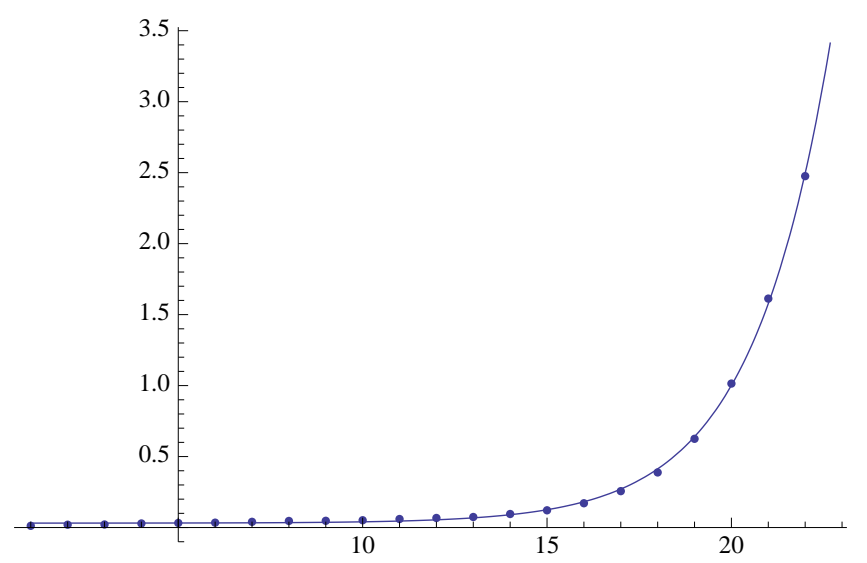

F34exp = NonlinearModelFit $\left[F 34\right.$ data $\left.[[1 ; ; 22]], b+d 0 * a^{\wedge} \mathbf{x},\{b, d 0, a\}, \mathbf{x}, \operatorname{MaxIterations} \rightarrow 1000\right]$

FittedModel $\left[0.0254402+0.0000485877 \ll 19 \gg^{x}\right.$

F34exp [ BestFit"]

$0.0254402+0.00004858771 .64621^{x}$

Show $[$ ListPlot $[F 34$ data $[[1 ; ; 22]], P l o t R a n g e \rightarrow A l l], P l o t[F 34 \exp [x],\{x, 1,25\}]]$

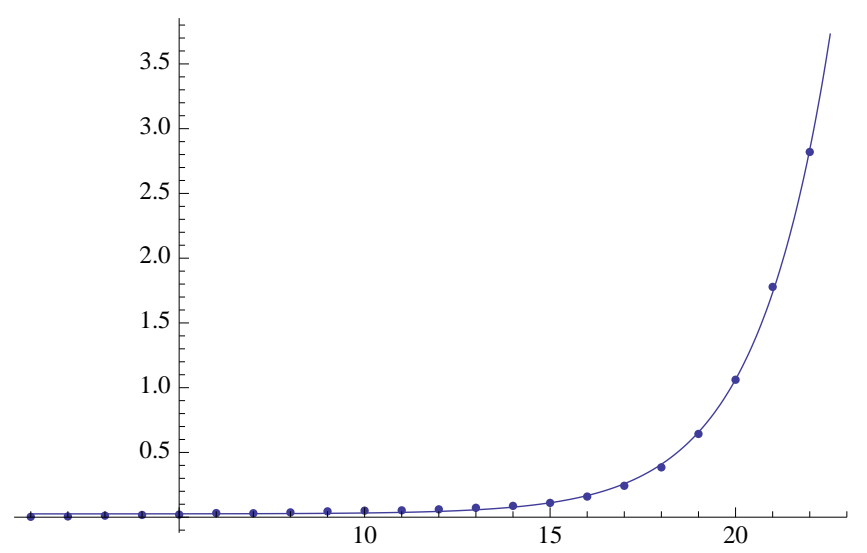

F41exp = NonlinearModelFit $\left[F 41\right.$ data $\left.[[1 ; ; 24]], b+d 0 * a^{\wedge} \mathbf{x},\{b, d 0, a\}, \mathbf{x}, \operatorname{MaxIterations} \rightarrow 1000\right]$ FittedModel $\left[0.0218628+1.48735 \times 10^{-6} \ll 19>^{x}\right]$

F41exp [ BestFit"]

$0.0218628+1.48735 \times 10^{-6} 1.74076^{x}$ 
Show [ListPlot [F41data $[1 ; ; 24]], P l o t R a n g e \rightarrow A l l], P l o t[F 41 \exp [x],\{x, 1,27\}]]$

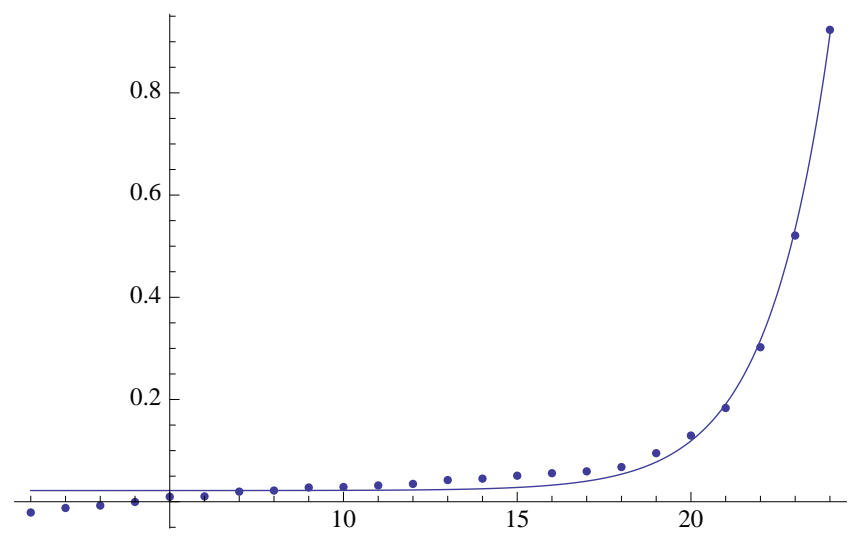

F42exp $=$ NonlinearModelFit $\left[F 42\right.$ data $\left.[[1 ; ; 24]], b+d 0 * a^{\wedge} \mathbf{x},\{b, d 0, a\}, \mathbf{x}, \operatorname{MaxIterations} \rightarrow 1000\right]$

FittedModel $\left[0.0247966+1.13024 \times 10^{-6} \ll 18 \gg^{x}\right.$

F42exp [ BestFit"]

$0.0247966+1.13024 \times 10^{-6} 1.77639^{x}$

Show [ListPlot [F42data $[[1 ; ; 24]], \mathrm{PlotRange} \rightarrow \mathrm{All}], \mathrm{Plot}[\mathrm{F42 \operatorname {exp }}[\mathbf{x}],\{\mathbf{x}, 1,27\}]]$

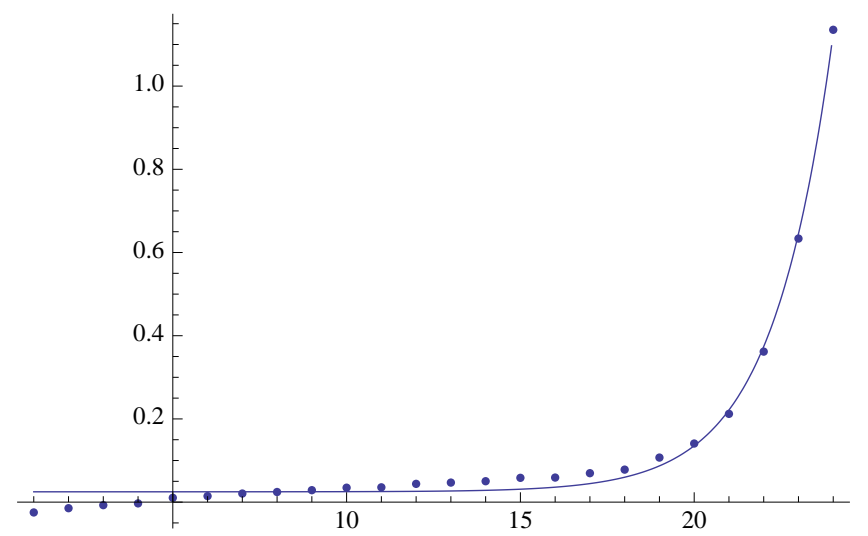

F43exp $=$ NonlinearModelFit $[F 43$ data $\left.[1 ; ; 24]], b+d 0 * a^{\wedge} \mathbf{x},\{b, d 0, a\}, \mathbf{x}, \operatorname{MaxIterations} \rightarrow 1000\right]$ FittedModel $\left[\left[0.0408012+8.62537 \times 10^{-6} \ll 19 \gg^{x}\right.\right.$

F43exp [ BestFit"]

$0.0408012+8.62537 \times 10^{-6} 1.62557^{x}$ 
Show [ListPlot $[F 43$ data $[1 ; ; 24]], P l o t R a n g e \rightarrow A l l], P l o t[F 43 \exp [x],\{x, 1,27\}]]$

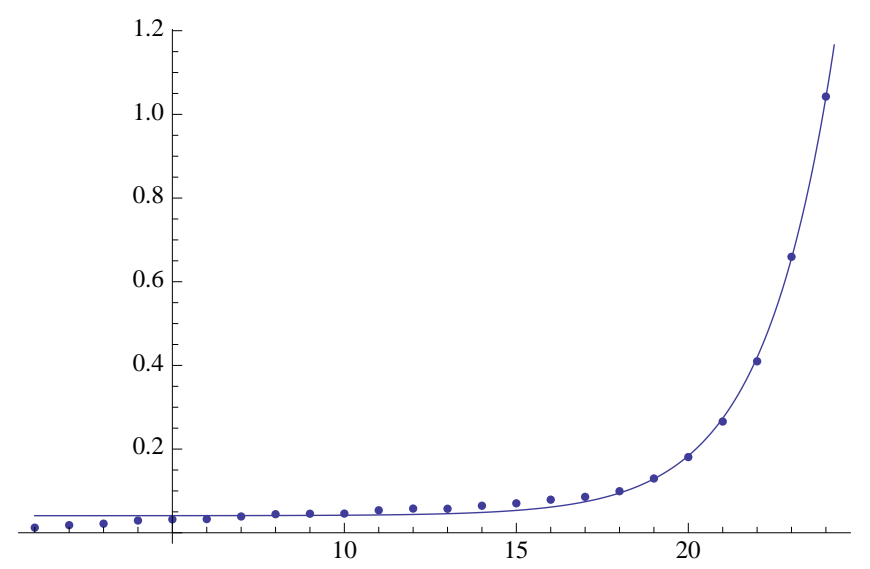

F44exp = NonlinearModelFit $\left[F 44\right.$ data $[[1 ; ; 24]], b+d 0 * a^{\wedge} \mathbf{x},\{b$, do, $\left.a\}, \mathbf{x}, \operatorname{MaxIterations} \rightarrow 1000\right]$

FittedModel $\left[0.0378632+3.7217 \times 10^{-6} \ll 19 \gg^{x}\right]$

F44exp [ BestFit"]

$0.0378632+3.7217 \times 10^{-6} 1.69894^{x}$

Show [ListPlot $[$ F44data $[1 ; ; 24]]$, PlotRange $\rightarrow$ All $], P l o t[F 44 \exp [x],\{x, 1,27\}]]$

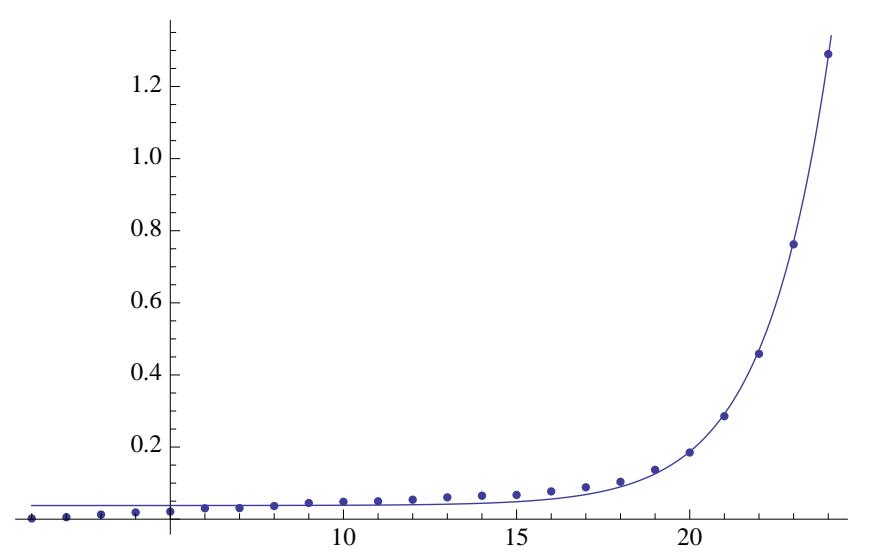

F51exp $=$ NonlinearModelFit $[$ F51data $[1 ; ; 28]], b+d 0 * a^{\wedge} \mathbf{x},\{b, d 0, a\}, \mathbf{x}$, MaxIterations $\left.\rightarrow 1000\right]$

FittedModel $\left[0.0299306+1.10969 \times 10^{-7} \ll 19 \gg^{x}\right.$

F51exp [ BestFit"]

$0.0299306+1.10969 \times 10^{-7} 1.80258^{x}$ 
Show [ListPlot $[F 51$ data $[1 ; ; 28]], P 1 o t R a n g e \rightarrow A l l], P 1 o t[F 51 \exp [\mathbf{x}],\{\mathbf{x}, 1,30\}]]$

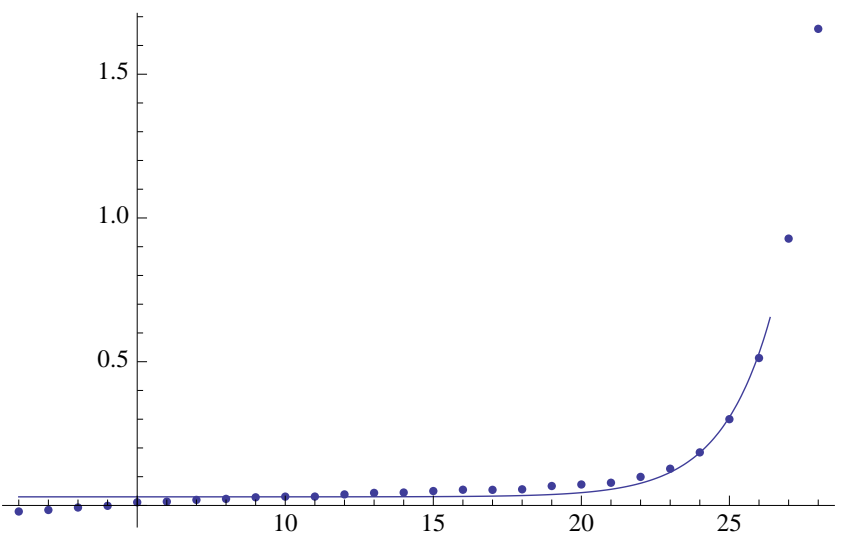

F52exp $=$ NonlinearModelFit [F52data $[[1 ; ; 28]], b+d 0 * a^{\wedge} \mathbf{x},\{b, d 0, a\}, \mathbf{x}$, MaxIterations $\left.\rightarrow 1000\right]$

FittedModel $\left[0.0301569+1.09252 \times 10^{-7} \ll 19 \gg^{x}\right.$

F52exp [ BestFit"]

$0.0301569+1.09252 \times 10^{-7} 1.80832^{x}$

Show [ListPlot [F52data $[1 ; ; 28]], \mathrm{PlotRange} \rightarrow \mathrm{All}], \mathrm{Plot}[\mathrm{F52 \operatorname {exp }}[\mathbf{x}],\{\mathbf{x}, 1,31\}]]$

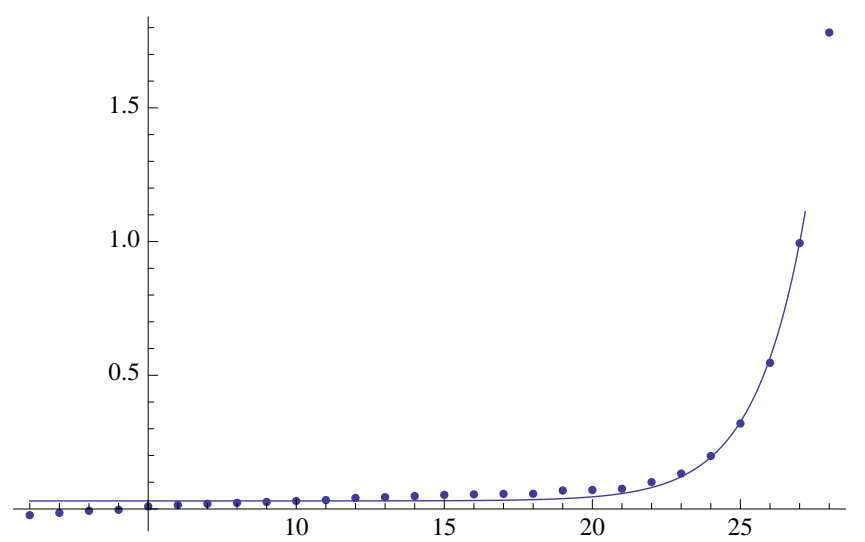

F53exp $=$ NonlinearModelFit $[$ F53data $\left.[1 ; ; 28]], b+d 0 * a^{\wedge} \mathbf{x},\{b, d 0, a\}, \mathbf{x}, \operatorname{MaxIterations} \rightarrow 1000\right]$

FittedModel $\left[0.0449902+2.02889 \times 10^{-6} \ll 19 \gg^{x}\right.$

F53exp [ BestFit"]

$0.0449902+2.02889 \times 10^{-6} 1.62525^{x}$ 
Show [ListPlot $[F 53$ data $[1 ; ; 28]]$, PlotRange $\rightarrow A l 1], P 1 o t[F 53 \exp [\mathbf{x}],\{\mathbf{x}, 1,31\}]]$

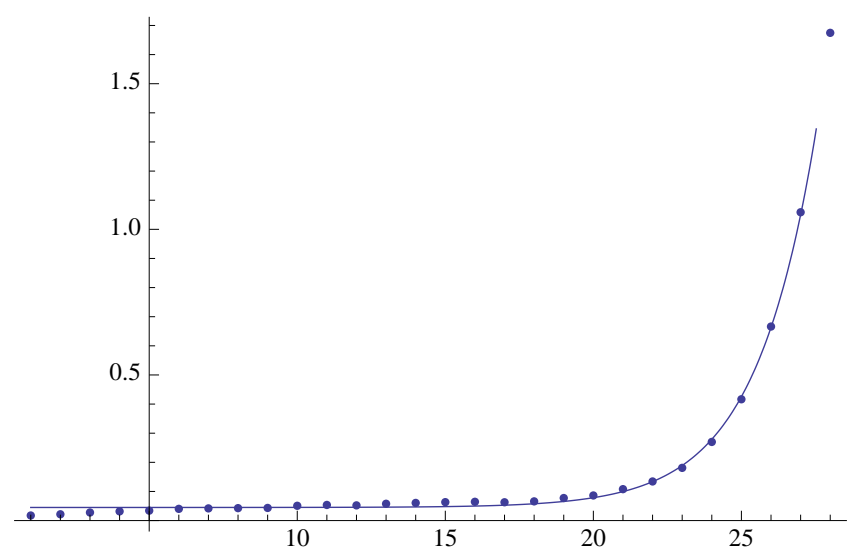

F54exp $=$ NonlinearModelFit $\left[F 54\right.$ data $\left.[[1 ; ; 28]], b+d 0 * a^{\wedge} \mathbf{x},\{b, d 0, a\}, \mathbf{x}, \operatorname{MaxIterations} \rightarrow 1000\right]$ FittedModel $\left[0.0398918+6.62153 \times 10^{-7} \ll 19 \gg^{x}\right.$

F54exp [ BestFit"]

$0.0398918+6.62153 \times 10^{-7} 1.68975^{x}$

Show [ListPlot $[F 54$ data $[[1 ; ; 28]]$, PlotRange $\rightarrow A l 1], P l o t[F 54 \exp [\mathbf{x}],\{\mathbf{x}, 1,31\}]]$

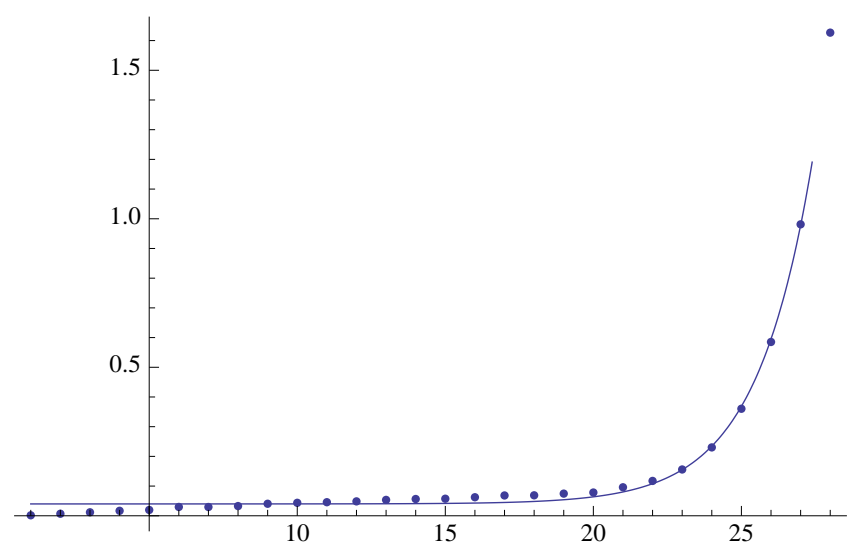

F61exp $=$ NonlinearModelFit $[F 61$ data $[1 ; ; 31]], b+d 0 * a^{\wedge} \mathbf{x},\{b$, d0, $a\}, \mathbf{x}$, MaxIterations $\left.\rightarrow 1000\right]$ FittedModel $\left[0.0347957+2.00307 \times 10^{-8} \ll 19 \gg^{x}\right]$

F61exp ["BestFit"]

$0.0347957+2.00307 \times 10^{-8} 1.78157^{x}$ 
Show [ListPlot $[\mathrm{F61data}[[1 ; ; 31]], \mathrm{PlotRange} \rightarrow \mathrm{All}], \mathrm{P} \operatorname{lot}[\mathrm{F61exp}[\mathbf{x}],\{\mathbf{x}, 1,35\}]]$

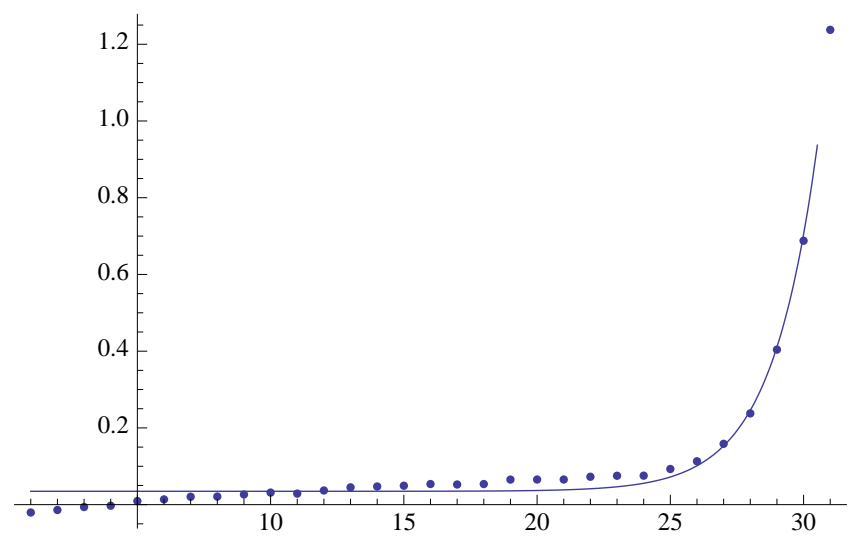

F62exp $=$ NonlinearModelFit $[F 62$ data $[1 ; ; 31]], b+d 0 * a^{\wedge} \mathbf{x},\{b, d 0, a\}, \mathbf{x}$, MaxIterations $\left.\rightarrow 1000\right]$ FittedModel $\left[0.037815+1.88923 \times 10^{-8} \ll 19 \gg^{x}\right]$

F62exp [ BestFit"]

$0.037815+1.88923 \times 10^{-8} 1.79139^{x}$

Show $[$ ListPlot $[F 62$ data $[[1 ; ; 31]], P l o t R a n g e \rightarrow A l l], P l o t[F 62 \exp [\mathbf{x}],\{\mathbf{x}, 1,35\}]]$

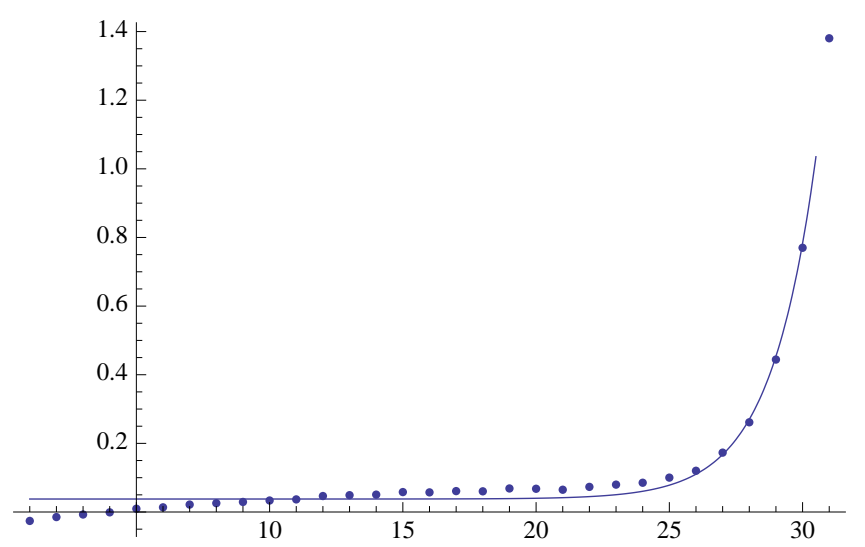

F63exp $=$ NonlinearModelFit $\left[\mathrm{F} 63\right.$ data $\left.[[1 ; ; 31]], b+d 0 * a^{\wedge} \mathbf{x},\{b, d 0, a\}, \mathbf{x}, \operatorname{MaxIterations} \rightarrow 1000\right]$ FittedModel $\left[0.0497411+3.7214 \times 10^{-7} \ll 19 \gg^{x}\right]$

F63exp [ BestFit"]

$0.0497411+3.7214 \times 10^{-7} 1.61823^{x}$ 
Show [ListPlot $[F 63$ data $[1 ; ; 31]], \mathrm{PlotRange} \rightarrow \mathrm{All}], \mathrm{Plot}[\mathrm{F63exp}[\mathbf{x}],\{\mathbf{x}, 1,35\}]]$

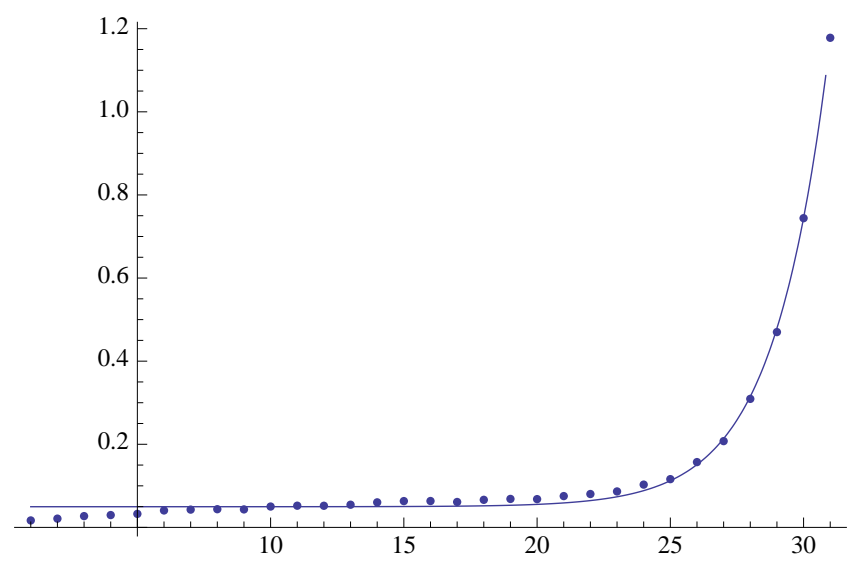

F64exp = NonlinearModelFit $\left[\mathrm{F} 64\right.$ data $\left.[[1 ; ; 31]], b+d 0 * a^{\wedge} \mathbf{x},\{b, d 0, a\}, \mathbf{x}, \operatorname{MaxIterations} \rightarrow 1000\right]$

FittedModel $\left[0.0488819+1.00182 \times 10^{-7} \ll 19 \gg^{\times}\right.$

F64exp [ BestFit"]

$0.0488819+1.00182 \times 10^{-7} 1.69067^{x}$

Show [ListPlot $[\mathrm{F} 64$ data $[[1 ; ; 31]], \mathrm{PlotRange} \rightarrow \mathrm{All}], \mathrm{Plot}[\mathrm{F} 64 \exp [\mathbf{x}],\{\mathbf{x}, 1,35\}]]$

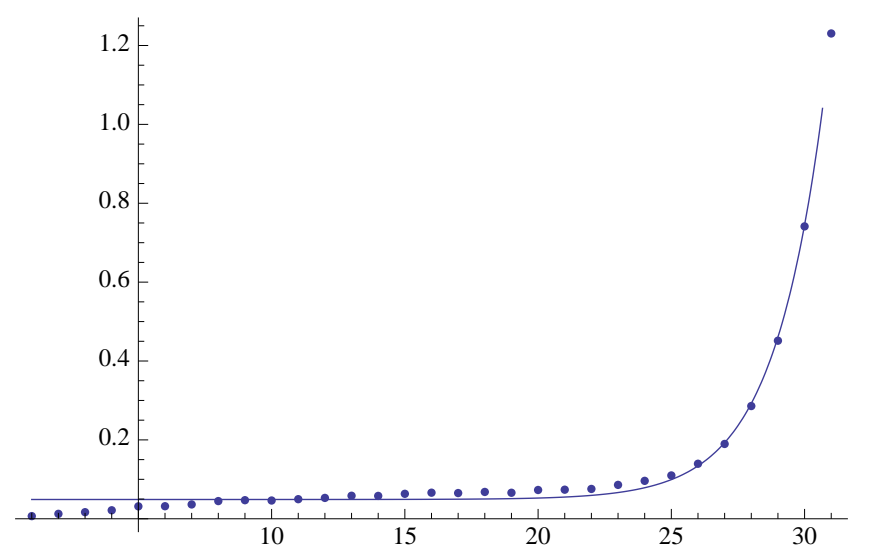

F71exp $=$ NonlinearModelFit $\left[F 71\right.$ data $[[1 ; ; 39]], b+d 0 * a^{\wedge} \mathbf{x},\{b, d 0, a\}, \mathbf{x}$, MaxIterations $\left.\rightarrow 1000\right]$

FittedModel $\left[0.0466201+2.5351 \times 10^{-10} \ll 19 \gg^{\times}\right]$

F71exp [ BestFit"]

$0.0466201+2.5351 \times 10^{-10} 1.76705^{x}$ 
Show [ListPlot [F71data $[1 ; ; 39]], \mathrm{PlotRange} \rightarrow \mathrm{All}], \mathrm{Plot}[\mathrm{F71} \exp [\mathbf{x}],\{\mathbf{x}, 1,46\}]]$

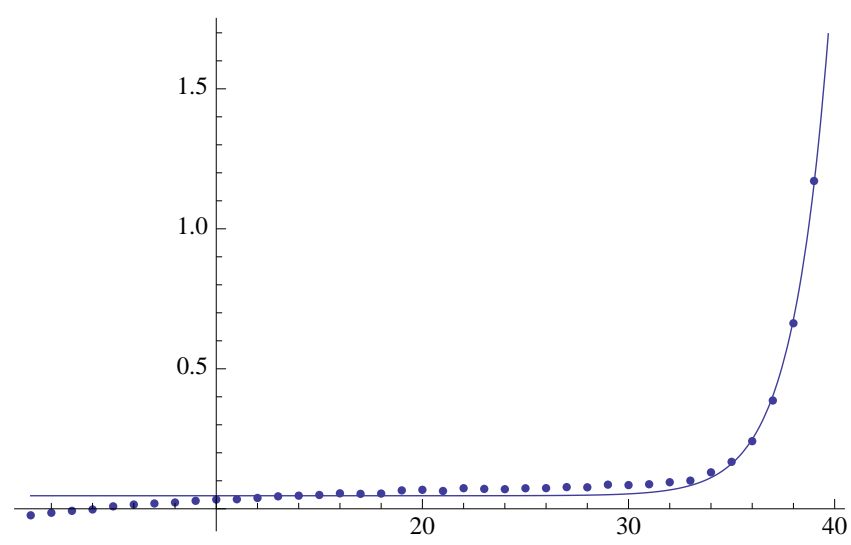

F72exp $=$ NonlinearModelFit [F72data $[1 ; ; 39]], b+d 0 * a^{\wedge} \mathbf{x},\{b, d 0, a\}, \mathbf{x}$, MaxIterations $\left.\rightarrow 1000\right]$

FittedModel $\left[0.0495575+2.2596 \times 10^{-10} \ll 18 \gg^{\times}\right]$

F72exp [ BestFit"]

$0.0495575+2.2596 \times 10^{-10} 1.77982^{x}$

Show [ListPlot $[F 72$ data $[[1 ; ; 39]]$, PlotRange $\rightarrow A l l], P l o t[F 72 \exp [x],\{x, 1,46\}]]$

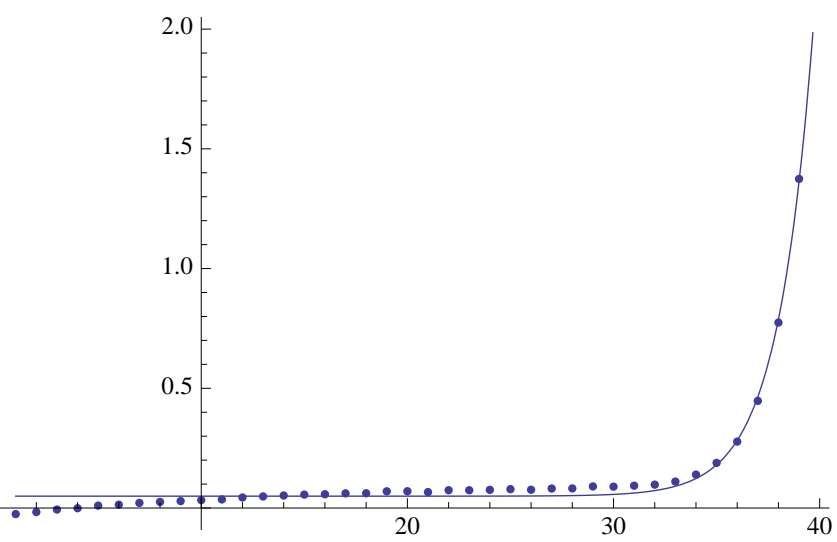

F73exp $=$ NonlinearModelFit $\left[F 73 d a t a\left[[1 ; 339], b+d 0 * a^{\wedge} \mathbf{x},\{b, d 0, a\}, \mathbf{x}\right.\right.$, MaxIterations $\left.\rightarrow 1000\right]$

FittedModel $\left[0.0607448+2.18005 \times 10^{-8} \ll 19 \gg^{\times}\right.$

F73exp [ BestFit"]

$0.0607448+2.18005 \times 10^{-8} 1.56834^{x}$ 
Show [ListPlot [F73data $[1 ; ; 39]]$, PlotRange $\rightarrow A l l], P l o t[F 73 \exp [\mathbf{x}],\{\mathbf{x}, 1,46\}]]$

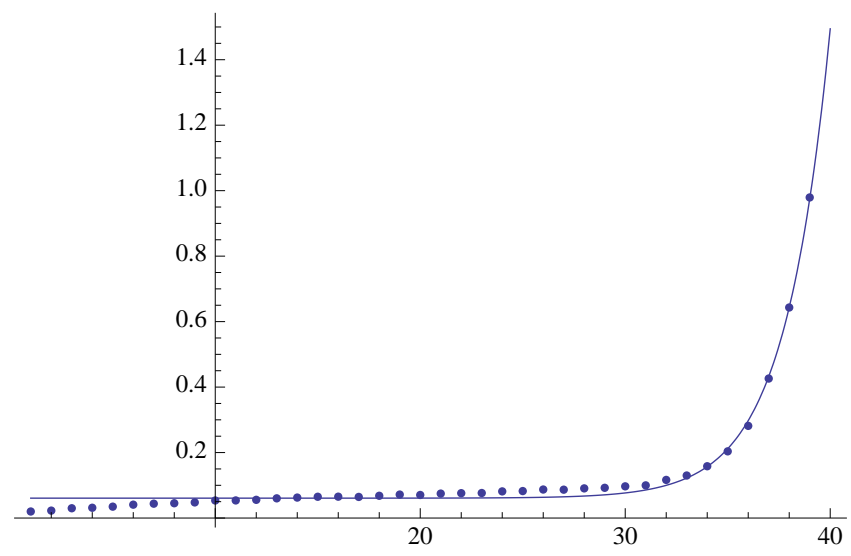

F74exp $=$ NonlinearModelFit $\left[F 74\right.$ data $[[1 ; ; 39]], b+d 0 * a^{\wedge} \mathbf{x},\{b, d 0, a\}, \mathbf{x}$, MaxIterations $\left.\rightarrow 1000\right]$

FittedModel $\left[\left[0.0568989+4.85376 \times 10^{-9} \ll 19 \gg^{x}\right.\right.$

F74 exp [ BestFit"]

$0.0568989+4.85376 \times 10^{-9} 1.64168^{x}$

Show [ListPlot [F74data $[1 ; ; 39]], \mathrm{PlotRange} \rightarrow \mathrm{All}], \mathrm{Plot}[\mathrm{F74 \operatorname {exp }}[\mathbf{x}],\{\mathbf{x}, 1,46\}]]$

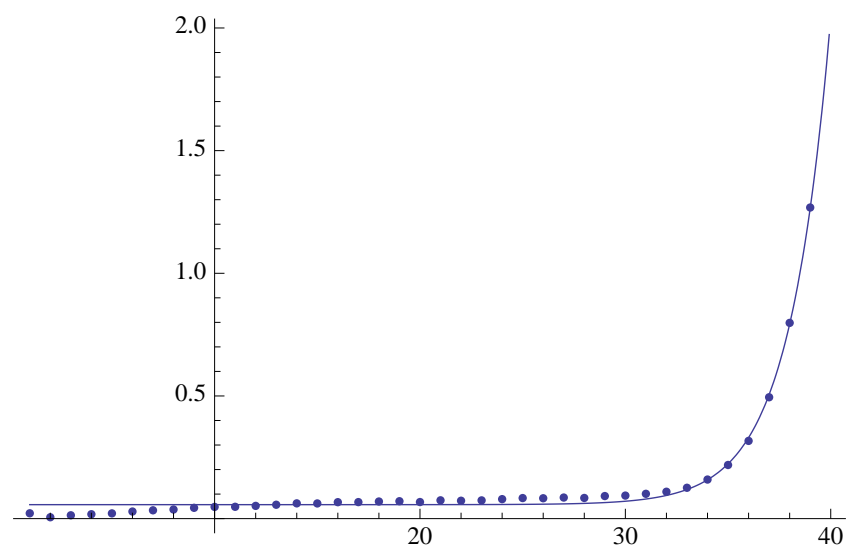

